Check for updates

Cite this: Mater. Chem. Front., 2020, 4, 1105

Received 15th November 2019, Accepted 23rd January 2020

DOI: 10.1039/c9qm00700h

rsc.li/frontiers-materials

\section{Recent progress in the syntheses and applications of multishelled hollow nanostructures}

\begin{abstract}
Maiyong Zhu, (D)* Jingjing Tang, Wenjing Wei and Songjun Li*
Multishelled hollow nanostructures have attracted considerable research interest owing to their unique structural features, promising properties, and fascinating performances in relevant applications. During the past few decades, considerable progress has been made in the synthesis of multishelled hollow nanostructures by accurately controlling their geometric morphology, chemical composition, thickness, number of shells, and their applications in various fields. In this review, we present a comprehensive overview on the recently used synthesis approaches for fabricating multishelled hollow nanostructures. For a comprehensive review, the synthesis approaches have been classified into four categories, namely, hard template, soft template, self-template, and template-free approaches based on the template/ structure-directing agent used. The advantages and disadvantages of each approach are discussed by comparing with each other. Furthermore, the fascinating performances of multishelled hollow nanostructures, application in energy conversion and storage, environment remediation, chemical catalysis, and biomedicine are comprehensively summarized. Combining certain typical examples and related theoretical analysis, the relationship between the structure of multishelled hollow nanomaterials and their specific application performances in the related areas is highlighted. Finally, the emerging challenges and future prospects of multishelled hollow nanostructures in the research and development for the future are outlined.
\end{abstract}

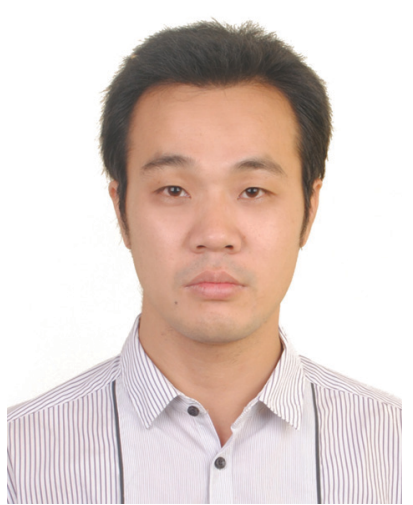

Maiyong Zhu
Maiyong Zhu received his PhD from Yangzhou University (2011), China. After that, he joined Jiangsu University (China) as an Assistant Professor to independently conduct research work. In 2015, he was promoted to be Associate Professor. His research interest covers several subjects in the field of advanced functional nanomaterials varying from noble metals, metal oxides/sulfides, carbon, conducting polymers, and metal-organic frameworks, which emphasizes the relationship among the synthesis, structure, and performance of functional materials. The application areas of the materials developed by his group include environmental treatment, chemical catalysis, and energy storage and conversion.

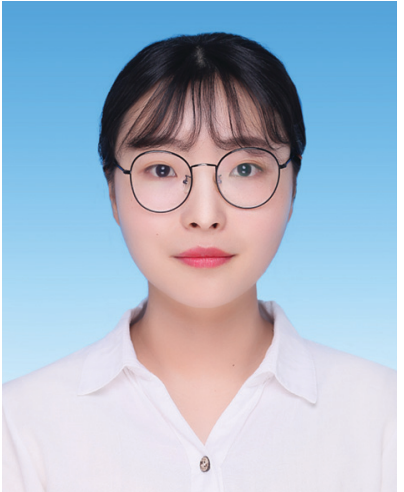

Jingjing Tang
Jingjing Tang received her bachelor degree in Materials Science from Jinling Institute of Technology (2017), China. Now, she is a graduate student under the supervision of $\mathrm{Dr}$ Maiyong $\mathrm{Zhu}$ at Materials Science \& Engineering, Jiangsu University. Her research work mainly focuses on the field of functional materials derived from metal-organic frameworks and their applications toward electrochemical energy storage. 


\section{Introduction}

Multishelled hollow nanostructures particularly refer to hollow structures that have at least two separate shells spatially ordered from the outside to the inside. As compared to solid materials as well as traditional single-shelled hollow nanostructures, multishelled hollow nanostructures exhibit multiple advantages. Firstly, multishelled structures may be endowed with prominent properties for specific applications by the integration of various functional components into a single system. Secondly, multishelled hollow structures are expected to allow more abundant exposure of the available active surface, which facilitates the accommodation of drug molecules and biomolecules (such as enzymes). Thirdly, the voids between the neighboring shells may act as a depot to hold various cargoes and a reaction chamber during catalysis. Finally, because of the higher number of shells, their mechanical stability is often much better than that of single-shelled hollow structures, which is particularly important when used as electrodes for energy storage, since such a void in hollow configurations as well as the space between the shells can be expected to buffer volume changes. ${ }^{1,2}$

Owing to their fascinating structural characteristics and superior properties, multishelled hollow nanostructures are expected to provide promising application potential in various fields. $^{3-8}$ The development of multishelled hollow nanostructures can be divided into two stages. Before 2009, owing to the shortage of facile, scalable, and controllable synthesis approaches, the development of multishelled hollow nanostructures was very slow. Since Wang's group developed the sequential template approach in 2009, the development of multishelled hollow nanostructures has been considerably enhanced. It is worth noting that other strategies have also been developed during the past years to synthesize multishelled hollow nanostructures in spite of the fact that about
$72.5 \%$ of multishelled hollow nanostructures have been prepared by this sequence template approach during $2018 .^{9}$ Many leading research groups have made pioneering works in the synthesis of multishelled hollow nanostructures. These methods could be generally divided into the hard template, soft template (various surfactants), self-template, and templatefree approaches. Each method has its own advantages and disadvantages. For example, the removal of a hard template without destroying the structure of the final product is usually a challenge for the hard template method. Meanwhile, the synthesis process for the hard template method is tedious and incurs a high cost. Soft templates (organic molecule assembly, such as micelles, emulsion droplets, and vesicles) are expected to yield products with uniform sizes and shapes. However, the purity of the final product might be seriously affected by the organic molecule assembly. Furthermore, organic molecule assemblies are thermodynamically unstable, which are highly sensitive to the environment; therefore, it is difficult to extend this method to a wide range of multishelled hollow structures. With regard to the self-template and templatefree methods, the quality of the final product may be controlled by adjusting the reaction parameters, such as temperature and time. However, these methods have considerable dependence on the precursor systems and reaction conditions. ${ }^{10-15}$

Certain research groups have summarized the exploration and development of multishelled hollow nanostructures. For example, a comprehensive review article in 2015 formulated by Qi et al. discussed the progress made in the synthesis of multishelled hollow nanostructures and their applications in dye-sensitized solar cells, lithium-ion batteries (LIBs), supercapacitors, sensors, photocatalysis, and drug delivery. ${ }^{16}$ Qin et al. provided an overview on the recent advances made in the synthesis of multishelled hollow nanostructures with typical singlecomponent metal oxides such as $\mathrm{NiO}, \mathrm{Co}_{3} \mathrm{O}_{4}$, and $\mathrm{ZnO}$, as well as their morphology-related applications. ${ }^{17}$ Ren et al. and Liu et al.

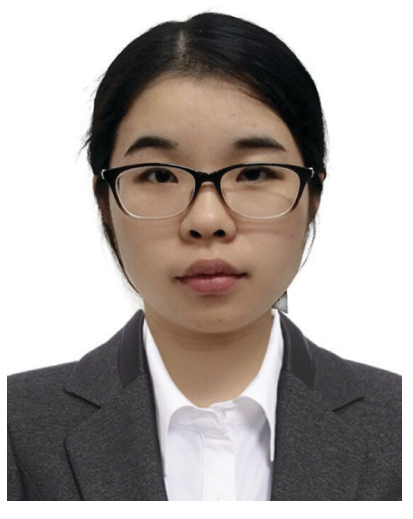

Wenjing Wei
Wenjing Wei is currently pursuing her PhD in School of Materials Science \& Engineering at Jiangsu University. She received her master degree from Jiangsu University in 2017. Her current research focuses on the catalytic application of smart materials and multishelled nanospheres.

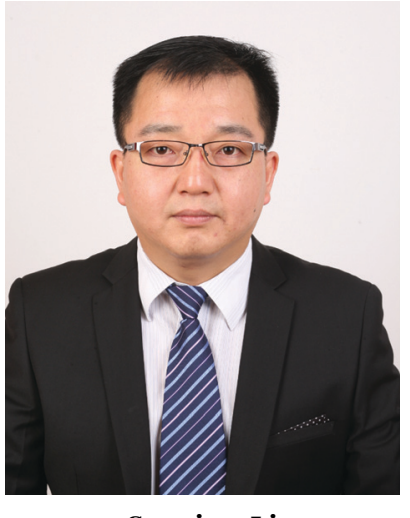

Songjun Li
Songjun $\mathrm{Li}$ received his $\mathrm{PhD}$ in Chemistry from Chengdu Institute of Organic Chemistry, Chinese Academy of Science (2005), China. After that, he joined Central China Normal University (China) as an Associate Professor to start his own research group. During 20082009, he was a postdoc fellow at University of Wisconsin, USA. In 2010-2011, he conducted his research work at Cranfield University (UK) under the support of Marie Curie Individual Fellowship. From 2012, he joined Jiangsu University (China) as a Distinguished Professor. In 2017, he was selected to be the Fellow of the Royal Society of Chemistry, UK. His research interests include the design and synthesis of smart polymeric materials for controllable and adjustable catalysis. 
summarized the research progress made in the synthesis of such materials and their promising applications in energy conversion and storage. ${ }^{18,19}$ Wang et al. gave a brief summary on the advancements made in multishelled hollow nanostructures for LIBs. ${ }^{20}$ Mao et al. highlighted the synthesis methods, particularly the sequential template approach, toward multishelled hollow nanostructures for various applications. ${ }^{9}$ Recently, Wang et al. also provided an in-depth understanding on the structure-performance correlation in multishelled hollow nanostructures for promising applications such as energy storage, electromagnetic wave absorption, catalysis, sensors, and drug delivery. ${ }^{21}$ Apparently, in spite of the above reviews on multishelled hollow nanostructures, most of which focused on certain special aspects, only Qi et al. provided a comprehensive review in 2015. It is well known that the research and development in this field has been very rapid. In this context, we have provided a more detailed discussion on the advancement of the syntheses and applications of multishelled hollow nanostructures. For the sake of convenience, the synthesis methods are divided into four categories: hard template method, soft template method, self-template method, and template-free method. For clearly showing the principle and operating procedures for each method, we provided many examples according to the template materials or precursors used for forming the multishelled hollow nanostructures. For instance, hard templates are further classified as silica, carbonaceous, polymer, metal oxide, and others. Soft templates involve single surfactant and dual/multi-surfactant systems. Three types of precursors were highlighted in the selftemplate approach section, namely, coordination polymers (CPs) or metal-organic frameworks (MOFs), metal glycerates/ glycorates, and metal-inorganic precursors (oxide/hydroxide/ carbonate). Here, it should be noted that the sequential template approach has not been listed as a specific synthesis method owing to its considerable overlap with the hard template and self-template methods. When compared with Qi's comprehensive review, additional recent application areas of multishelled hollow nanostructures have been incorporated into this review, such as the adsorptive removal of pollutants, catalytic conversion of gas pollutants, and high-performance batteries (sodium-ion batteries; lithium-sulfur batteries). Finally, a brief outlook and future challenges of this research field are also pointed out.

\section{Synthesis strategies for multishelled hollow nanostructures}

\subsection{Hard template approach}

From a simplistic point of view, the hard template approach seems to be the most straightforward tool for synthesizing multishelled hollow nanostructures. In general, the conversion of a traditional hard template approach to multishelled hollow nanostructures includes the following: (i) the fabrication of templates with the desired shapes; (ii) in situ formation of the target architectures using the above templates, yielding template@target materials with core-shell structures; (iii) assembly

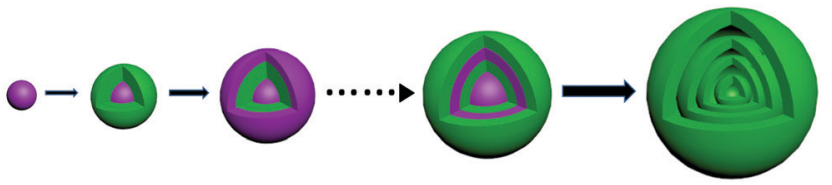

Fig. 1 Schematic illustration of the hard template principle for preparing multishelled hollow nanostructures.

of the template on the outer surface of the template@target composites; and (iv) repeating steps ii and iii, and ending with step ii, and finally resulting in the removal of the template (Fig. 1). To form a successful coating between the shell and template materials, a surface modification step might become necessary, using which the surface functionality can be adjusted, such as surface charge and polarity. Many techniques, such as solvothermal treatment, static electronic deposition, electrochemical deposition, chemical oxidation, or self-assembly process, might be useful for depositing the shell/template materials on the template/shell surface. With regard to the removal of the hard template, a specific procedure might considerably depend on the inherent properties of the hard template. Typically, chemical etching, thermal decomposition or calcination, and solvent dissolution in a specific solvent can be applied for the removal of the hard template. Until now, several rigid materials, such as silica, carbonaceous, polymers, and metallic oxides, have been successfully developed as hard templates to synthesize multishelled hollow nanostructures. In this section, we will introduce the hard template syntheses of multishelled hollow nanostructure based on their different compositions.

2.1.1 Silica-based hard templates. Silica is one of the most widely used hard template materials, either for core support or as an intermediate layer between the shells for the synthesis of multishelled hollow nanostructures owing to its easy fabrication and derivatization. Hwang et al. reported a traditional shell-by-shell assembly approach to synthesize multishelled porous $\mathrm{TiO}_{2}$ hollow nanoparticles using silica colloids as the hard template (Fig. 2a). ${ }^{19,22}$ The fabricated $\mathrm{SiO}_{2} @ \mathrm{TiO}_{2}$ coreshell nanoparticles (ST CS NPs) have an easily modifiable surface, allowing further functionalization of polyvinylpyrrolidone (PVP) to form ST/PVP CS NPs. It has been well demonstrated that PVP-modified inorganic nanoparticles can be further functionalized by $\mathrm{SiO}_{2}$ through the traditional Stöber process using tetraethyl orthosilicate (TEOS) as a silicon source, as fabricated in the $\mathrm{SiO}_{2} @ \mathrm{TiO}_{2} @ \mathrm{SiO}_{2} \mathrm{CS}$ NPs material. The repetition followed by the deposition of $\mathrm{TiO}_{2}$ and $\mathrm{SiO}_{2}$ may generate more-layered structures comprising $\mathrm{SiO}_{2}$ and $\mathrm{TiO}_{2}$. The multishelled porous $\mathrm{TiO}_{2}$ hollow nanoparticles without silica were obtained from the anatase crystalline phase of $\mathrm{TiO}_{2}$ by calcination at $900{ }^{\circ} \mathrm{C}$ followed by chemical etching with $\mathrm{NaOH}$ solution. By precisely adjusting the experimental conditions, three hollow samples with different numbers of shells, i.e., single-shelled, double-shelled, and triple-shelled hollow nanoparticles, were obtained (Fig. 2b-d). The TEM images of all the samples clearly reveal the shell location and their hollow structure. As the procedure implies, multishelled 
a

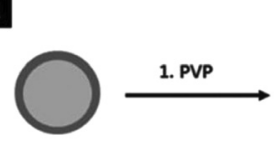

ST CS NPS

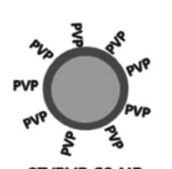

ST/PVP CS NPS
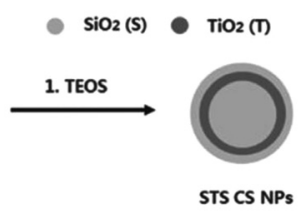

STS CS NPS

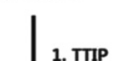

1. TTIP

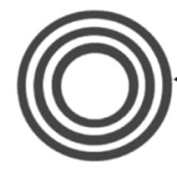

Multi-shell HNPs

b

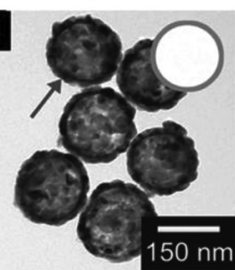

STSTST CS NPs

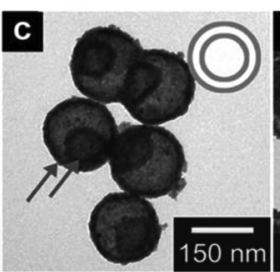

1. PVP/TEOS 2. TTIP

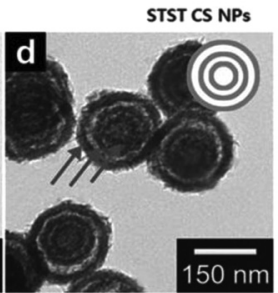

Fig. 2 (a) Schematic illustration of the synthesis of multishelled porous $\mathrm{TiO}_{2}$ hollow nanoparticles using $\mathrm{SiO}_{2}$ as the hard template. Typical TEM images of single-shelled (b), double-shelled (c), and triple-shelled (d) hollow $\mathrm{TiO}_{2}$ nanospheres. Reprinted from ref. 19 with permission. Copyright 2019 John Wiley \& Sons.

structures were fabricated by iterations of $\mathrm{SiO}_{2}$ - and $\mathrm{TiO}_{2}$-coating processes. Intuitively, the outermost diameter should increase with the number of shells and the innermost diameter should be the same since the innermost $\mathrm{SiO}_{2}$ core template is the same; interestingly, the outermost diameters of all these samples were almost the same $(\sim 160 \mathrm{~nm})$. This may be due to the volume shrinkage in the $\mathrm{TiO}_{2}$ shell upon the removal of the $\mathrm{SiO}_{2}$ template. The location of the inner shells is not always at the center of the hollow nanoparticles, as observed in the TEM images, since the inner shells can move in the voids between the shell layers.

Lou and co-workers presented a shell-by-shell hydrothermal synthesis process to synthesize doubled-shelled hollow $\mathrm{SnO}_{2}$ using monodisperse $\mathrm{SiO}_{2}$ nanospheres (diameter: $\sim 320 \mathrm{~nm}$ ) as the sacrificial hard template. ${ }^{23}$ The core silica template was coated with a double layer of polycrystalline $\mathrm{SnO}_{2}$ obtained via a hydrothermal process to form uniform double shells. Subsequently, the silica-cores-based as-formed double-shelled particles were etched with dilute HF to control its size (partially dissolved or completely dissolved). Interestingly, it was observed that the double-shelled hollow particles transformed into single-shelled particles on etching with the dilute HF solution.

In addition to solid silica-based materials, hollow silica structures have also been employed as the hard template to synthesize multishelled hollow nanostructures. When using a hollow template, both inner and outer surfaces could adsorb the precursors of the target materials. Hierarchical doubleshelled metal sulfide (NiS, CuS, and MnS) hollow structures were successfully synthesized by $\mathrm{Yu}$ et al. by using silica nanoboxes as the hard template. ${ }^{24}$ The synthesis strategy for the formation of multishelled NiS box-in-box hollow structures started from silica boxes (shell thickness: $\sim 100 \mathrm{~nm}$ ) that were presynthesized using $\mathrm{Fe}_{2} \mathrm{O}_{3}$ as the template. Initially, the silica boxes were dispersed in an alkaline solution containing nickel acetate, $\mathrm{NH}_{3} \cdot \mathrm{H}_{2} \mathrm{O}$, and $\mathrm{NH}_{4} \mathrm{Cl}$. Under hydrothermal conditions, silica gets partially dissolved to generate silicate anions. These alkaline-induced silicate anions would further react with $\mathrm{Ni}^{2+}$, forming a thin layer of Ni silicate on the surface of the silica box. As the reaction proceeds, more silicate anions derived from the dissolution of silica may diffuse outward and promote the growth of the Ni silicate shell, simultaneously forming a void between the residual silica core and Ni silicate shell. When the size of the void reaches a critical value, the silicate anions may react with the inward-diffusing $\mathrm{Ni}^{2+}$, forming a secondary shell layer of $\mathrm{Ni}$ silicate. After the silica core is completely consumed, a double-shelled Ni silicate box-in-box hollow structure is formed. Finally, by treating with $\mathrm{Na}_{2} \mathrm{~S}$, the as-prepared $\mathrm{Ni}$ silicate box-in-box hollow structures with double shells are converted into Nis box-in-box hollow nanostructures. Such a synthesis procedure is expected to be extended to synthesize other metal sulfides with similar structures. Fig. 3a-c show the typical TEM images of the as-prepared box-in-box hollow nanostructures of NiS, CuS, and MnS, which exhibits uniform box-in-box hollow structures of all the synthesized materials. Owing to the different properties of these different metal ions, the primary building blocks of the three box-in-box hollow structures are ultrathin nanosheets (NiS), ultrafine nanoneedles (CuS), and nanoparticles (MnS). Du et al. prepared double-shelled NiS hollow nanostructures with different morphologies (cube, ellipsoid, and capsule) using similar procedures. ${ }^{25}$ Using hollow silica as the hard template, Li et al. prepared double-shelled $\mathrm{SnO}_{2} @ \mathrm{C}$ hollow spheres. ${ }^{26}$

Sun et al. prepared double- and quadruple-shelled carbon hollow spheres by using single-shelled silica and doubleshelled silica, respectively, as a permeable template. The entire preparation procedure is shown in Fig. $4 .{ }^{27}$ Evidently, step I enumerates the process to form double-shelled carbon hollow spheres, which involves several procedures. Initially, mesoporous silica hollow spheres were obtained by using a common vesicle template route, as explained in an earlier report. In order to improve the affinity between the carbon (precursor) and mesoporous silica hollow spheres, the amine-terminated functionalization properties of the mesoporous silica hollow spheres were applied. Subsequently, both the outer and inner surfaces of the mesoporous silica hollow spheres were coated
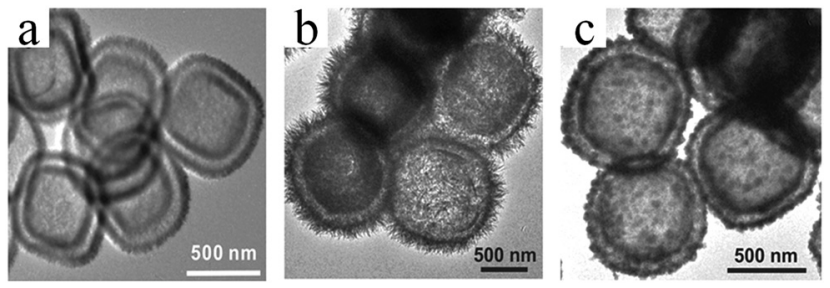

Fig. 3 Schematic illustration of the synthesis procedure of NiS box-inbox hollow structures. TEM images of the box-in-box hollow structures: NiS (a), CuS (b), and MnS (c). Reprinted from ref. 24 with kind permission. 


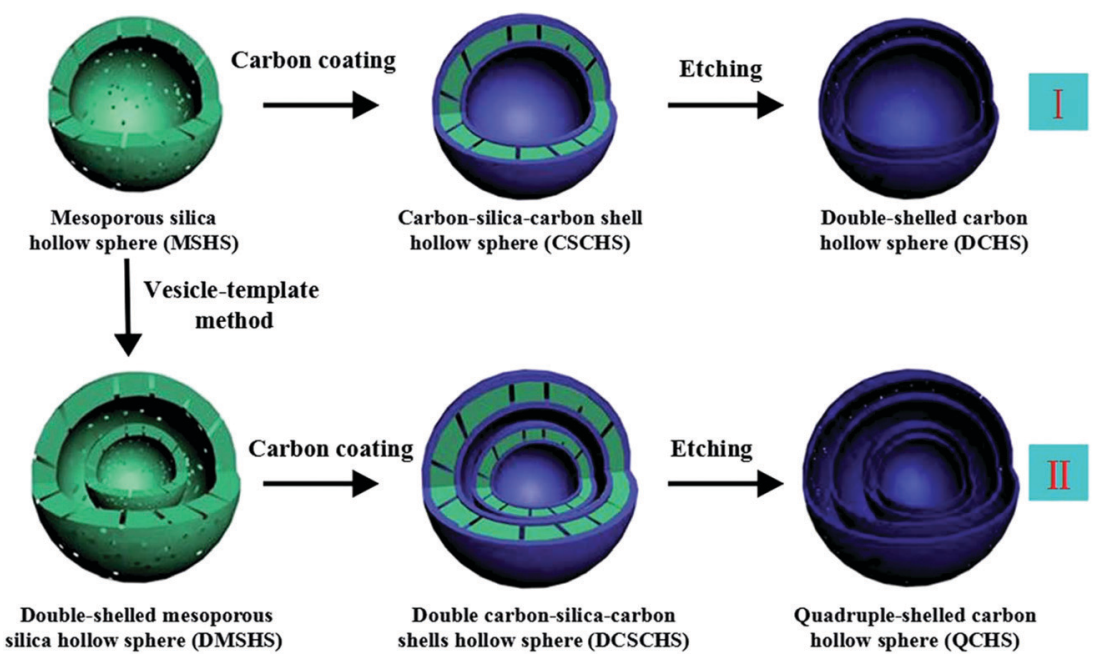

Fig. 4 Schematic illustration of the preparation procedure of double- and quadruple-shelled carbon hollow spheres. Reproduced from ref. 27 with permission

with carbon using glucose as a precursor through a hydrothermal reaction followed by calcination. Finally, double-shelled carbon hollow spheres were obtained after removing the mesoporous silica hollow spheres with HF. The procedures for forming quadruple-shelled carbon hollow spheres (step II) are very similar to those for forming double-shelled carbon hollow spheres by replacing the mesoporous silica hollow spheres with doubleshelled silica hollow spheres.

2.1.2 Carbonaceous-based hard templates. Carbonaceous materials are another widely used hard template candidate for the formation of diverse functionalized materials with desirable structures. It is well known that most of the available carbonaceous materials are derived from organic molecules (such as glucose, sucrose, polymers, and so on), ensuring that carbonaceous materials usually possess numerous functional groups, such as amino, hydroxide, and carboxylic acid. These functional groups show excellent affinity toward metal ions; therefore, carbonaceous materials offer a promising prospect in the fabrication of various metal-containing functional materials, as well as multishelled metal oxide hollow nanostructures.

Lai $e t$ al. developed a generic sequential template approach based on carbonaceous microspheres to prepare a series of multishelled metal oxide hollow microspheres, as shown in Fig. 5a. The driving force for the adsorption of metal cations into the carbonaceous template is the electrostatic attraction between the positively charged cations and negatively charged carbonaceous templates. After the adsorption of metallic ions, the carbonaceous spheres (CSs) could be sacrificed under thermal treatments, thereby yielding metal oxides with hollow structures. By repeating the adsorption of metal precursors and the heating process, the number of shells of the final metal oxide product may be controlled. As shown in Fig. $5 b-d$, the number of shells of $\alpha-\mathrm{Fe}_{2} \mathrm{O}_{3}$ hollow microspheres can be easily controlled, ranging from 2 to $4 .^{28} \mathrm{Wu}$ et al. and Chu et al. demonstrated the shell-by-shell assembly formation mechanism of synthesizing multishelled $\mathrm{Cr}_{2} \mathrm{O}_{3}, \mathrm{ZnO}, \mathrm{NiO}$, and $\mathrm{Co}_{3} \mathrm{O}_{4}$ hollow a
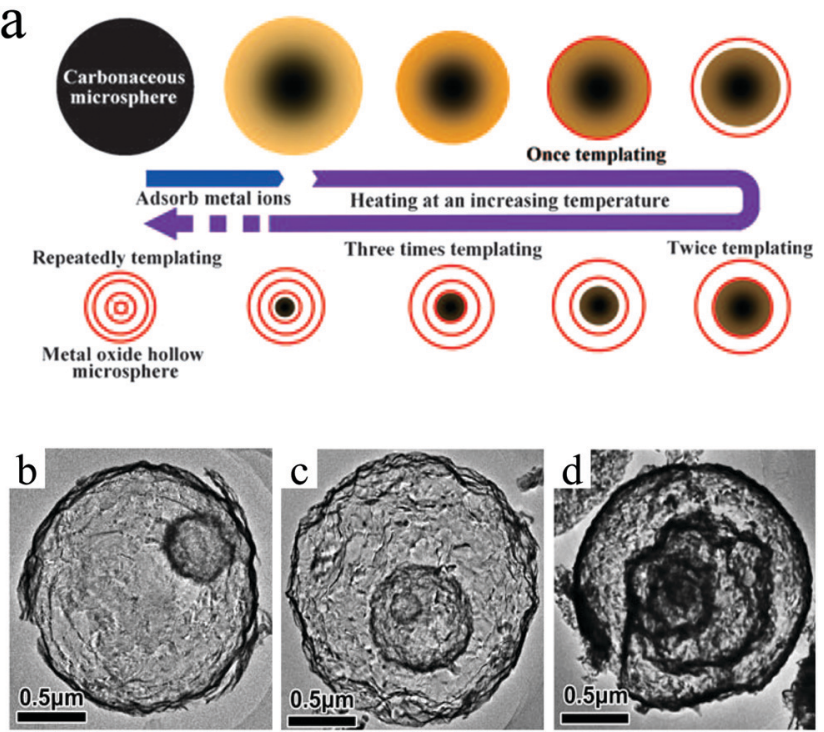

Fig. 5 (a) Schematic diagram of the sequential template strategy to multishelled metal oxide hollow microspheres. TEM images of doubleshelled (b), triple-shelled (c), and quadruple-shelled (d) $\alpha-\mathrm{Fe}_{2} \mathrm{O}_{3}$ hollow microspheres. Reprinted from ref. 28 with kind permission.

microspheres by using glucose-derived carbonaceous microspheres as the hard template. Interestingly, the metallic precursor $\left(\mathrm{Cr}\left(\mathrm{NO}_{3}\right)_{3} \cdot 9 \mathrm{H}_{2} \mathrm{O}, \mathrm{ZnSO}_{4} \cdot 7 \mathrm{H}_{2} \mathrm{O}, \mathrm{Ni}\left(\mathrm{NO}_{3}\right)_{2} \cdot 6 \mathrm{H}_{2} \mathrm{O}\right.$, or $\left.\mathrm{Co}\left(\mathrm{NO}_{3}\right)_{2} \cdot 6 \mathrm{H}_{2} \mathrm{O}\right)$ and carbonaceous precursor (D-glucose) were initially mixed together before the carbonaceous template formation and subsequent annealing treatment. ${ }^{29-33}$

Dong et al. presented a programmable heating process to synthesize multishelled $\mathrm{ZnO}, \mathrm{TiO}_{2}$, and $\alpha-\mathrm{Fe}_{2} \mathrm{O}_{3}$ hollow microspheres using carbonaceous microspheres as the hard template. $^{34-36}$ The formation mechanism of ZnO multishelled hollow microspheres is shown in Fig. 6. Hydroxide- or carboxylic-acid-modified CSs can easily absorb zinc ions when immersed in a zinc salt solution. Upon calcination, the CSs can 


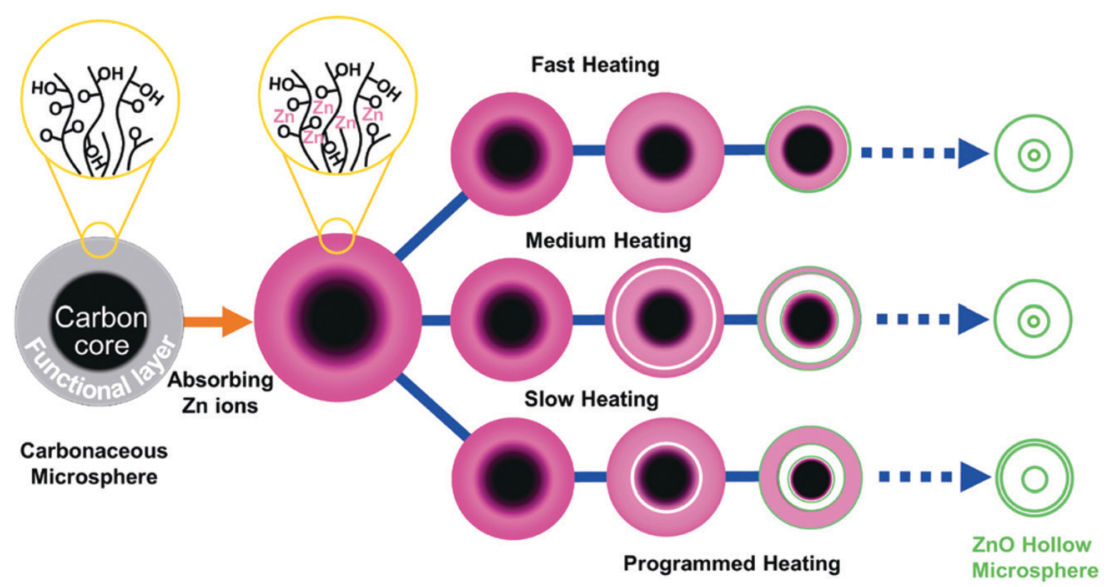

Fig. 6 Schematic illustration of the synthesis process of multishelled ZnO hollow microspheres. Reprinted from ref. 34 with kind permission.

evaporate and direct the fabrication of $\mathrm{ZnO}$ shells. The separation of the outer $\mathrm{ZnO}$ shell layer and contraction of the inner carbon core are the two key factors to generate multishelled ZnO hollow nanospheres. Moreover, the concentration of zinc precursors also has a significant influence on the structure of the final products. Typically, the higher concentration of zinc precursors may facilitate a deeper penetration of zinc ions into the carbonaceous template cores, which implies that additional zinc ions will get adsorbed on the inside of the inner core. As a consequence, the number of shells may increase with a higher concentration of zinc precursors. In addition, the diameter of the carbonaceous template is another important factor affecting the quality of the final products. It is well known that the larger carbonaceous microspheres are expected to provide more space to accommodate more zinc ions, thereby forming a hollow structure with more shells (quadruple or even more). Notably, other experimental parameters besides the above may also play a significant role in controlling the number of shells and thickness, such as the time for which the carbonaceous template is immersed in the zinc salt solution, acidity $(\mathrm{pH}$ value) of the solution, and experimental temperature, although the authors did not perform such investigations. In addition, suffering from the limited ability of the loading metal ion of the unmodified carbonaceous microsphere template, the synthesis approach could not be extended to prepare other multishelled semiconductor hollow structures with thick exterior shells. Using similar procedures, Niu et al. prepared $\mathrm{SnO}_{2}$ hollow spheres with double and triple shells. ${ }^{37}$
In order to enhance the interaction between the carbonaceous template and metallic ions as well as improve the loading capability of the template, the pretreatment of a carbonaceous hard template is often applied. Dong et al. further reported the preparation of multishelled $\mathrm{SnO}_{2}$ hollow microspheres with controllable numbers of shells $(\sim 1-5)$ using alkali-treated carbonaceous microspheres as the hard template through a direct one-step thermal treatment. ${ }^{38}$ The alkali-treated carbonaceous templates have abundant negatively charged hydroxyl surface functional groups, increasing the absorption of $\mathrm{Sn}^{4+}$ cation in the templates. Therefore, an increased number of $\mathrm{SnO}_{2}$ shells could be formed during such thermal treatments. Meanwhile, closed exterior double shells may be formed at higher absorption amounts of $\mathrm{Sn}^{4+}$. By varying the dosage of $\mathrm{Sn}^{4+}$, the number of multishells in the final product can be controlled to be within 1-5; the corresponding TEM images are shown in Fig. 7.

Despite the considerable achievement of controlling the number of shells, further improved measurements should be performed to synthesize a wide range of materials. When the radius of the hydrate metal ions is larger, such as cobalt, it might be difficult to control the number of shells in the product by using the above generic method. In order to circumvent this issue, on the basis of systematically investigating the relationship between the size and diffusion rate of the hydrate metal ions, Wang and co-workers successfully controlled the number of shells of $\mathrm{Co}_{3} \mathrm{O}_{4}$ hollow microspheres. According to their preliminary experiments, it was proposed that the rapid

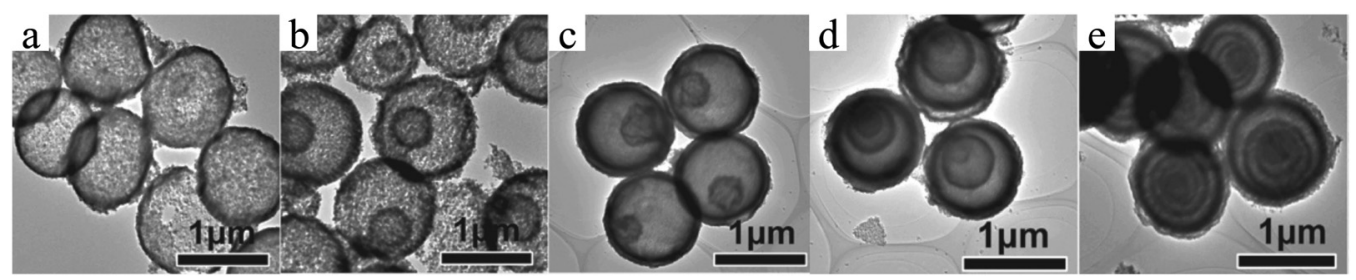

Fig. 7 TEM images of (a) single-, (b) double-, (c) triple-, (d) quadruple-, and (e) quintuple-shelled hollow $\mathrm{SnO}_{2}$ microspheres prepared by a one-step thermal treatment process using alkali-treated carbonaceous microspheres as the hard template. Reprinted from ref. 38 with kind permission. 


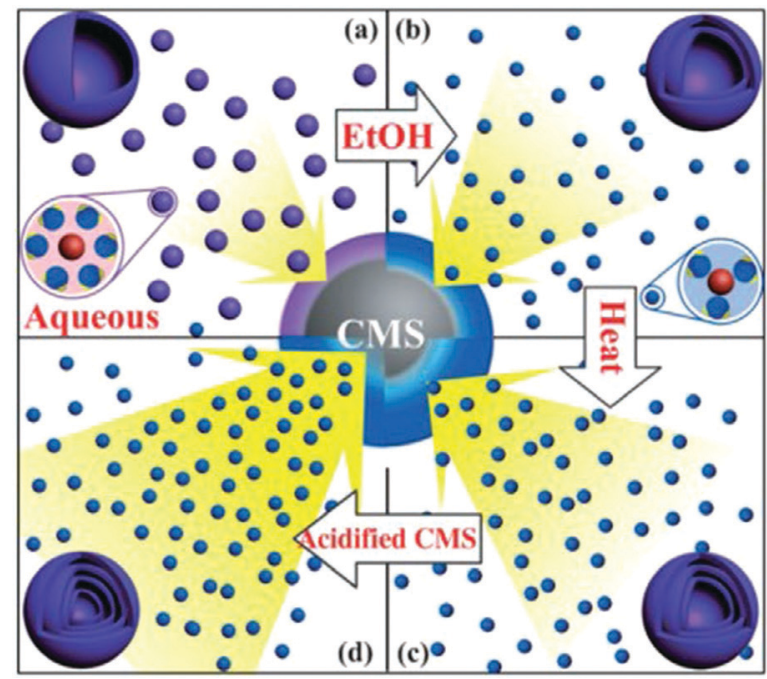

Fig. 8 Mechanism for the formation of multishelled $\mathrm{CO}_{3} \mathrm{O}_{4}$ hollow microspheres under different adsorption conditions. Reprinted from ref. 39 with permission.

diffusion rate of hydrate metal ions facilitates the formation of more shelled structures, while both the size of the hydrate metal ions and temperature are important factors affecting their diffusion rates. Accordingly, a modified carbonaceous template approach in which the solvent composition could be adjusted was presented to control the number of shells in the $\mathrm{Co}_{3} \mathrm{O}_{4}$ hollow microspheres, as shown in Fig. $8 .^{39,40}$ In an aqueous medium, $\mathrm{Co}$ (II) ions are expected to be in the form of $\left[\mathrm{Co}\left(\mathrm{H}_{2} \mathrm{O}\right)_{6}\right]^{2+}$ (Fig. 8a), which has a large radius as compared to normal ions and relatively low diffusion rates in a carbonaceous microsphere template; therefore, only single-shelled $\mathrm{Co}_{3} \mathrm{O}_{4}$ hollow microspheres (Fig. 9a) can be formed after the removal of the carbonaceous template via calcination. In a mixture of water and ethanol (e.g., ethanol/water in equal volume of $1: 1$ ) as the solvent, $\mathrm{Co}(\mathrm{II})$ ion would exist as $\left[\mathrm{Co}\left(\mathrm{H}_{2} \mathrm{O}\right)_{(6-x)}\right]^{2+}$, where $x=3-6$ (Fig. 8b). With a decrease in the number of coordination water molecules, the radius of $\mathrm{Co}$ (II) decreases, offering a rapid diffusion rate than that in $\left[\mathrm{Co}\left(\mathrm{H}_{2} \mathrm{O}\right)_{6}\right]^{2+}$, which ensures that an increased amount of cobalt ions gets loaded into the carbonaceous template; this yields double-shelled $\mathrm{Co}_{3} \mathrm{O}_{4}$ hollow microspheres (Fig. 9b). By further increasing the ethanol content in the solvent $(75 \%, \mathrm{v} / \mathrm{v})$ along with simultaneously heating to a higher temperature (Fig. 8c), a higher diffusion rate can be achieved, thereby generating the ready adsorption of $\mathrm{Co}$ (II) by means of carbonaceous microspheres. As a consequence, a triple-shelled $\mathrm{Co}_{3} \mathrm{O}_{4}$ hollow microsphere is obtained (Fig. 9c). In addition to adjusting the solvent component, the authors also investigated the effect of acidtreated template on the adsorption of $\mathrm{Co}(\mathrm{II})$ as well as on the final structure of $\mathrm{Co}_{3} \mathrm{O}_{4}$ (Fig. 8d). Their results demonstrated that the carbonaceous microsphere template, after the treatment of acid, offered a much larger specific surface area and enhanced pore volume than those of the template that did not undergo any acid treatment. Larger surface areas and enhanced pore volumes are expected to increase the loading of metal ions, yielding quadruple-shelled hollow microspheres (Fig. 9d). In the subsequent work, they extended this sequence template approach to prepare multishelled hollow $\left(\mathrm{Co}_{2 / 3} \mathrm{Mn}_{1 / 3}\right)\left(\mathrm{Co}_{5 / 6} \mathrm{Mn}_{1 / 6}\right)_{2} \mathrm{O}_{4}$. By precisely controlling the experimental parameters, the number of shells could be effectively controlled and the maximum number of shells reached up to a septuple, which is the highest number of shells obtained until now. ${ }^{41}$ Further, Wang et al. synthesized nanorod-assembled multishelled $\mathrm{Co}_{3} \mathrm{O}_{4}$ microspheres in a mixture of water and ethanol as the solvent using a carbon microsphere as the hard template, where carbon microspheres@ $\mathrm{Co}_{2} \mathrm{CO}_{3}(\mathrm{OH})_{2}$ core-shell composites were formed with a subsequent calcination process. After the calcination treatment, the carbon microspheres@ $\mathrm{Co}_{2} \mathrm{CO}_{3}(\mathrm{OH})_{2}$ core-shell composites could be converted into multishelled $\mathrm{Co}_{3} \mathrm{O}_{4}$ microspheres. $^{42}$

We have noted that the acid treatment on a carbonaceous template may promote the loading of cobalt ions into the template; in their earlier report, the treatment of alkali facilitated the adsorption of $\mathrm{Sn}^{4+}$ by the carbonaceous microsphere template. In fact, both acid treatment and alkali treatment may activate the carbonaceous template, generating more functional surface groups. According to the electronic theory of acids and alkalis, Co(II) ions can easily donate an electron due their large size and low nuclear charge, while the case for Sn(Iv) is completely opposite. As a result, acid-treated carbonaceous microspheres are more preferable for loading Co(II), whereas alkali-treated carbonaceous microspheres are more suitable for adsorbing $\operatorname{Sn}(\mathrm{IV})$.

In another work, Wang et al. demonstrated that the $\mathrm{pH}$ value of a solution may also significantly affect the formation of multishelled $\mathrm{Mn}_{2} \mathrm{O}_{3}$ hollow nanostructures in addition to adjusting the solvent components and temperature. ${ }^{43}$ The higher $\mathrm{pH}$ of
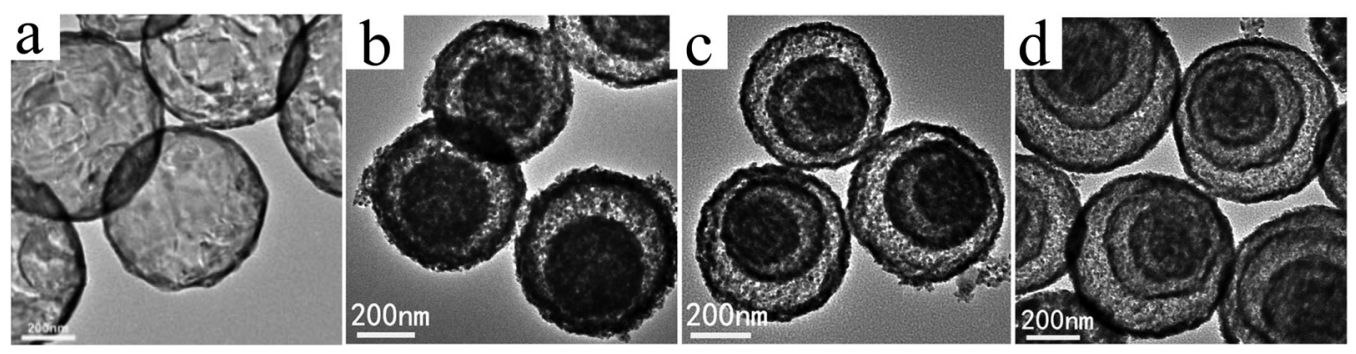

Fig. 9 TEM images of (a) single-, (b) double-, (c) triple-, and (d) quadruple-shelled $\mathrm{CO}_{3} \mathrm{O}_{4}$ hollow microspheres. Reprinted from ref. 40 with kind permission. 
aqueous manganese acetate solutions can lead to the fabrication of hollow $\mathrm{Mn}_{2} \mathrm{O}_{3}$ spheres with a higher number of shells. The reason for such phenomena could be explained as follows. The higher concentration of $\mathrm{H}^{+}$in the solution means that the functional groups (mainly hydroxide groups) get protonated. Therefore, a decrease in $\mathrm{pH}$ results in a decrease in the surface negative charge of the carbonaceous microsphere template, which could be confirmed by its zeta potential. As a consequence, the amount of Mn(II) adsorbed on the template also decreases, which is not beneficial for the formation of multiple-shelled hollow nanostructures. In particular, single-, double-, and triple-shelled $\mathrm{Mn}_{2} \mathrm{O}_{3}$ hollow nanospheres could be obtained at the $\mathrm{pH}$ values of $3.35,4.43$, and 6.43 , respectively, of the solution. It should be noted that only a limited amount of Mn(II) could be adsorbed onto the carbonaceous microsphere templates, generating $\mathrm{Mn}_{2} \mathrm{O}_{3}$ nanoparticles, but not $\mathrm{Mn}_{2} \mathrm{O}_{3}$ hollow structures, when the $\mathrm{pH}$ of the solution was less than 1. Moreover, the form of $\mathrm{Mn}$ (II) may vary with the $\mathrm{pH}$ of the solution. It is well known that $\mathrm{Mn}$ (II) is likely to exist in the form of $\left[\mathrm{Mn}\left(\mathrm{H}_{2} \mathrm{O}\right)_{6-x} \mathrm{Cl}_{x}\right]^{2-x}(0<x<2$, when $0 \leq \mathrm{pH} \leq 7)$ when $\mathrm{HCl}$ is used to adjust the $\mathrm{pH}$ of the solution. At lower $\mathrm{pH}$, more $\mathrm{Cl}^{-}$can coordinate with $\mathrm{Mn}$ (II), which implies that the electrostatic attraction between $\mathrm{Mn}$ (II) and carbonaceous template becomes weaker and hence the amount of adsorbed $\mathrm{Mn}$ (II) onto the carbonaceous template decreases due to the generation of $\mathrm{Mn}_{2} \mathrm{O}_{3}$ hollow microspheres with a limited number of shells (even nanoparticle shape) after heating. Tian et al. proposed a general and versatile strategy to prepare a wide range of multishelled metal oxide hollow microspheres, including, but not limited to, $\mathrm{Co}_{3} \mathrm{O}_{4}, \mathrm{CuO}, \mathrm{Fe}_{2} \mathrm{O}_{3}, \mathrm{In}_{2} \mathrm{O}_{3}$, $\mathrm{PrO}_{1.83}$, and $\mathrm{ZnO}$, by using a commercial gluconate salt as both a carbon and metal precursor, where a similar effect of $\mathrm{pH}$ value could be observed. ${ }^{44}$

Using glucose as the carbon source and nickel nitrate as the nickel source, Chu et al. systematically investigated the effect of $\mathrm{pH}$ of the solution (ranging from 8.3 to 10.9) on the morphology of $\mathrm{NiO}$ using $\mathrm{NH}_{3} \cdot \mathrm{H}_{2} \mathrm{O}$ as the $\mathrm{pH}$ regulator. ${ }^{45}$ In their work, glucose was initially dehydrated to generate the carbon spheres. The surface of such carbon is hydrophilic and attached to numerous $\mathrm{OH}^{-}$groups. Consequently, this carbon sphere can act as a substrate for ion penetration in the subsequent reaction processes. Their investigations demonstrated that multishelled NiO hollow spheres could be finally formed only at high $\mathrm{pH}$ values, such as 10.9. This result could be explained as follows. At high pH values, the $\mathrm{OH}^{-}$and $\mathrm{NH}_{3}$ concentrations, chelating interactions between $\mathrm{NH}_{3}$ and $\mathrm{Ni}^{2+}$, and growth rate of $\mathrm{Ni}(\mathrm{OH})_{2}$ nuclei would simultaneously increase, leading to the gradual formation of $\mathrm{Ni}(\mathrm{OH})_{2}$ nuclei, self-organized, and further reacted. Meanwhile, there would be a large number of $\mathrm{OH}^{-}$on the surface of the carbon spheres; therefore, it is likely to form $\mathrm{Ni}(\mathrm{OH})_{2}$ nuclei on the surface of the carbon spheres. These $\mathrm{Ni}(\mathrm{OH})_{2}$ nuclei would further assemble into nanoparticles on the basis of the coalescence mechanism. Finally, the inside-out Ostwald ripening growth mechanism would dominate, yielding multishelled structures. After the calcination treatment, the carbon spheres would decompose and $\mathrm{Ni}(\mathrm{OH})_{2}$ would dehydrate to afford NiO. The possible mechanism of the formation is shown in Fig. 10.

Considering most of the existing approaches are only available in the synthesis of simple binary multishelled metal oxide hollow spheres, Zhang et al. developed a general penetrationsolidification-annealing approach to synthesize multicomponent metal oxide hollow spheres with multishelled structures using CSs as the hard template, as shown in Fig. $11 \mathrm{a} .{ }^{46}$ The entire process comprises the following steps. Firstly, the carbonaceous hard template spheres were synthesized via the hydrothermal carbonization of glucose at $\sim 160-180{ }^{\circ} \mathrm{C}$ and then dispersed into ethylene-glycol-containing metallic acetate (such as nickel acetate, cobalt acetate, and/or manganese acetate). Secondly, heating treatment at $120{ }^{\circ} \mathrm{C}$ was performed to promote the penetration of metallic ions deep into the carbonaceous template. This procedure could last for $12 \mathrm{~h}$. In the next step, the substance was further heated up to $170{ }^{\circ} \mathrm{C}$ for $2 \mathrm{~h}$ till the metal glycolate was formed on both the inner and outer surfaces of the carbonaceous template. With the consumption of ethylene glycol, the viscosity of the system considerably increased; therefore, the process was referred to as solidification. Finally, in order to obtain multishelled mixed metal oxide hollow spheres, the decomposition of metal glycolate and removal of carbonaceous template were achieved by annealing at $500{ }^{\circ} \mathrm{C}$ for $4 \mathrm{~h}$. Fig. 11b-g shows the typical TEM images of some of the obtained samples. Evidently, triple-shelled hollow spheres could be fabricated for $\mathrm{CoMn}_{2} \mathrm{O}_{4}, \mathrm{Co}_{1.5} \mathrm{Mn}_{1.5} \mathrm{O}_{4}, \mathrm{MnCo}_{2} \mathrm{O}_{4}, \mathrm{ZnMn}_{2} \mathrm{O}_{4}$, $\mathrm{ZnCo}_{2} \mathrm{O}_{4}$, and $\mathrm{NiCo}_{2} \mathrm{O}_{4}$. The thickness of these mixed metal oxides slightly varied with a combination of metals within $\sim 30-50 \mathrm{~nm}$. By varying the diameter of the employed carbonaceous template or adjusting the penetration time, the number of shells could be adjusted. Generally, the larger diameter of the utilized carbonaceous template was expected to provide more space to form more shells in the final hollow structures. Such a result is in good

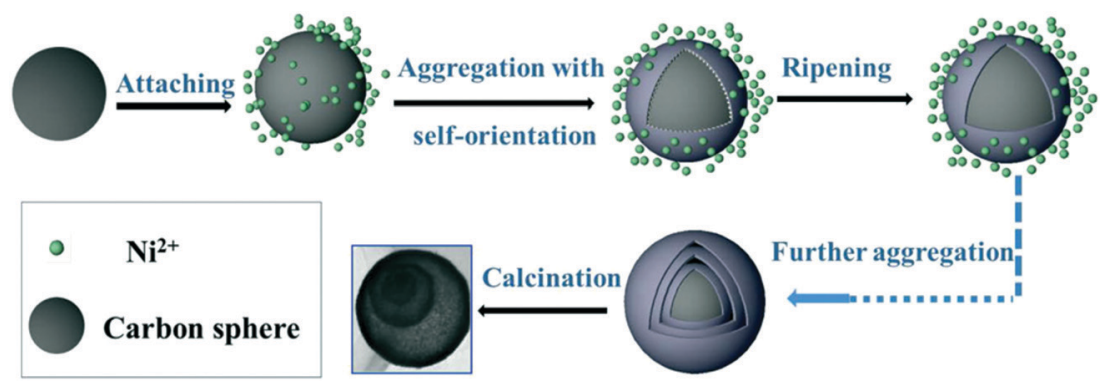

Fig. 10 Plausible formation process of multishelled NiO microspheres. Reproduced from ref. 45 with permission. 
a (I) Penetration
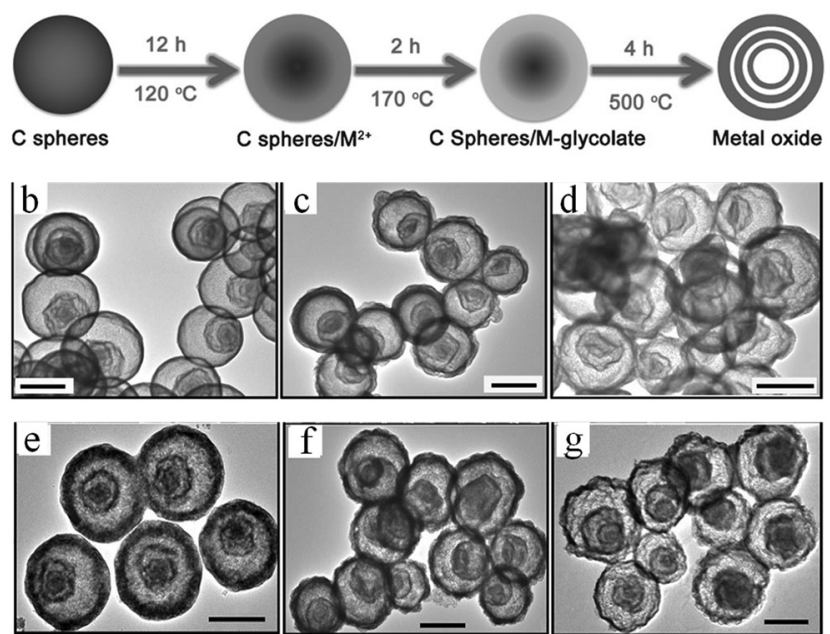

Fig. 11 (a) Preparation schematics of multicomponent metal oxide multishelled hollow spheres via the penetration-solidification-annealing approach using carbon spheres as the hard template. Typical TEM images with scale bars of $500 \mathrm{~nm}$ for some of the samples of multicomponent metal oxide multishelled hollow spheres. (b) $\mathrm{CoMn}_{2} \mathrm{O}_{4}$, (c) $\mathrm{CO}_{1.5} \mathrm{Mn}_{1.5} \mathrm{O}_{4}$, (d) $\mathrm{MnCO}_{2} \mathrm{O}_{4}$, (e) $\mathrm{ZnMn}_{2} \mathrm{O}_{4}$, (f) $\mathrm{ZnCO}_{2} \mathrm{O}_{4}$, and (g) $\mathrm{NiCO}_{2} \mathrm{O}_{4}$. Reprinted from ref. 46 with kind permission.

agreement with those obtained from earlier reports. ${ }^{34}$ Zhang et al. used a similar principle to prepare $\mathrm{CoFe}_{2} \mathrm{O}_{4}$ hollow spheres. ${ }^{47}$ Recently, Peng et al. proposed an adsorption-calcinationreduction approach to prepare various spinel oxides $\left(\mathrm{NiCo}_{2} \mathrm{O}_{4}\right.$, $\mathrm{CoMn}_{2} \mathrm{O}_{4}$, and $\mathrm{NiMn}_{2} \mathrm{O}_{4}$ ) with necklace-like multishelled hollow structures using carbonaceous microspheres as the sacrificial template. It should be pointed out that the goal of the additional reduction treatment was to induce the formation of oxygen vacancies in the spinel crystals, which may boost the performance of the materials and various properties, such as catalytic activity. ${ }^{48}$

As a versatile template, carbonaceous microspheres could adsorb either metallic cations or metal-containing anions. The first example of using a carbonaceous microsphere to adsorb an anion for preparing multishelled hollow nanostructures was reported by Wang et al. In their epoch-making work, a series of multishelled $\left(\mathrm{V}_{2} \mathrm{O}_{5}, \mathrm{MnO}_{2}, \mathrm{MoO}_{3}, \mathrm{Cr}_{2} \mathrm{O}_{3}\right.$, and $\left.\mathrm{WO}_{3}\right)$ hollow nanostructures were obtained. The formation mechanism involved the chemical adsorption of metal-containing anions $\left(\mathrm{VO}_{3}{ }^{-}, \mathrm{MnO}_{4}{ }^{-}, \mathrm{MoO}_{4}{ }^{2-}, \mathrm{CrO}_{4}{ }^{2-}\right.$, and $\mathrm{W}_{7} \mathrm{O}_{24}{ }^{6-}$ ) onto carbonaceous microsphere templates followed by a Trojan catalytic combustion process to remove the carbonaceous template. The driving force for the adsorption of metal anions into the microspheres is different from the traditional cation-adsorption process. Taking $\mathrm{VO}_{3}{ }^{-}$as the example, because oxygen (O) prefers to coordinate with $\mathrm{V}^{5+}$ by donating its $\mathrm{p}$ electrons to the empty $\mathrm{d}$ orbits of $\mathrm{V}^{5+}$, the electrostatic repulsion energy can be considered to be much smaller than the binding energy between $\mathrm{VO}_{3}{ }^{-}$with the $\mathrm{OH}$ group via a coordination interaction. As a result, the adsorption of $\mathrm{VO}_{3}{ }^{-}$onto the negatively charged carbonaceous template with abundant oxygen-containing groups is thermodynamically feasible via coordination bonds. Meanwhile, in real-world cases, the penetrated cations (such as $\mathrm{NH}_{4}^{+}$) can partly neutralize the negative charges, promoting the further adsorption of metal-containing anions. ${ }^{49}$ On the basis of their systematic work, the same group recently extended this principle to the co-absorption of positive and negative ions to fabricate binary metal oxides $\left(\mathrm{Fe}_{2}\left(\mathrm{MoO}_{4}\right)_{3}, \mathrm{NiMoO}_{4}, \mathrm{MnMoO}_{4}\right.$, $\mathrm{CoWO}_{4}, \mathrm{MnWO}_{4}$, etc.) with multishelled hollow structures. ${ }^{50}$ Zong et al. prepared $\mathrm{YVO}_{4}$ multishelled hollow spheres using a yttrium CS as the hard template. ${ }^{51}$ By means of a hydrothermal route, yttrium CSs were firstly prepared, which possessed hydrophilic functional groups. Therefore, $\mathrm{VO}_{4}{ }^{3-}$ anions could easily absorb to form the $\mathrm{YVO}_{4}$ complex oxide. The multishelled hollow spheres of $\mathrm{YVO}_{4}$ with uniform morphology, high crystallinity, and controlled number of shells (up to three) could be successfully obtained by thermal annealing.

In certain cases, CSs may not only serve as a grid template to form multishelled hollow target materials, but also take part in the reaction process. For example, Zhang and co-workers prepared double-shelled $\mathrm{MnO}_{2} / \mathrm{CeO}_{2}-\mathrm{MnO}_{2}$ hollow spheres using colloidal CSs as the hard template, as shown in Fig. 12a. ${ }^{52}$ The negatively charged carbonaceous microspheres were firstly applied as the substrate to deposit Ce species by means of a layerby-layer self-assembly process, forming core-shell $\mathrm{CSs}_{\mathrm{CeO}_{2}}$ precursor spheres (Fig. 12b). Based on the fact that there were plenty of mesoporous pores in the shell layer of the $\mathrm{CeO}_{2}$ precursor, a $\mathrm{KMnO}_{4}$ solution was percolated through the $\mathrm{CeO}_{2}$ precursor that had deeper access to the spheres. Subsequently, the redox reactions between the carbonaceous core $/ \mathrm{KMnO}_{4}$ and $\mathrm{CeO}_{2}$ precursor/ $\mathrm{KMnO}_{4}$ occurred, generating core-shell CSs@ $\mathrm{MnO}_{2} / \mathrm{CeO}_{2}-\mathrm{MnO}_{2}$ due to the close contact between the CSs core and Ce species. Owing to the Kirkendall effect caused by the diffusion couple of the faster inward-diffusing $\mathrm{MnO}^{4-}$ and outward-diffusing organic carbon chains, the core-shell-structured CSs@ $\mathrm{MnO}_{2} / \mathrm{CeO}_{2}-\mathrm{MnO}_{2}$ could be easily converted into yolk-shell CSs@ $\mathrm{MnO}_{2} / \mathrm{CeO}_{2}-\mathrm{MnO}_{2}$ (Fig. 12c). Finally, annealing the above yolk-shell structure in air, the residual CS core would completely decompose, thereby generating $\mathrm{MnO}_{2} / \mathrm{CeO}_{2}-\mathrm{MnO}_{2}$ double-shelled hollow spheres (Fig. 12d).
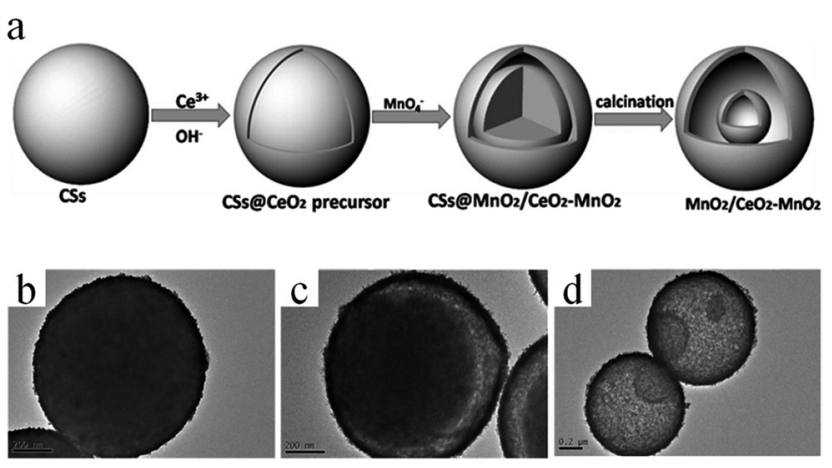

Fig. 12 (a) Schematic illustration of the synthesis process of $\mathrm{MnO}_{2} /$ $\mathrm{CeO}_{2}-\mathrm{MnO}_{2}$ double-shelled hollow spheres. TEM images of (b) $\mathrm{CSs} \mathrm{aCeO}_{2}$ precursor core-shell spheres, (c) yolk-shell CSs $\mathrm{MnO}_{2} / \mathrm{CeO}_{2}-\mathrm{MnO}_{2}$, and (d) $\mathrm{MnO}_{2} / \mathrm{CeO}_{2}-\mathrm{MnO}_{2}$ double-shelled hollow spheres. Reprinted from ref. 52 with kind permission. 
In addition to the abovementioned merits of a carbonaceous template that make it more resistant to acids and alkalis as compared to a silica template, the former consumes a lesser amount of solvent or additional chemicals, except precursors, in the entire preparation process. It should be noted that the carbonaceous template may not be available for producing metallic oxides in a sub-stable oxidation state (such as $\mathrm{Cu}_{2} \mathrm{O}$, $\mathrm{Fe}_{3} \mathrm{O}_{4}$, and $\mathrm{MnO}$ ), since in most cases, the removal of carbon is usually carried out by annealing in an air flow. These sub-stable compounds may be oxidized by $\mathrm{O}_{2}$ in air. Further, high energy consumption in the removal of the carbonaceous template for the simultaneous formation of multishelled hollow nanostructures may be another disadvantage of the carbonaceous template approach.

2.1.3 Polymer-based hard templates. Polystyrene (PS) is one of the most widely used hard template candidates for preparing various hollow nanostructures; this is because the synthesis of PS is fairly simplistic. Meanwhile, it is easy to control the particle sizes and surface functional groups for PS. For instance, Yang et al. reported an efficient strategy to fabricate double-shelled inorganic hollow spheres (Fig. 13a). ${ }^{53}$ The first sulfuric-acid-functionalized PS hollow spheres were obtained by the reaction of PS hollow spheres with $\mathrm{H}_{2} \mathrm{SO}_{4}$. Then, the inorganic metal ion precursors would be absorbed into both the inner and outer surfaces of sulfonated hollow spheres to form multilayered composite hollow spheres. At last, the core PS could be removed by $\mathrm{N}, \mathrm{N}$-dimethylformamide (DMF). Fig. 13b and c show the typical double-shelled $\mathrm{TiO}_{2}$ and $\mathrm{Fe}_{3} \mathrm{O}_{4}$ hollow spheres. Apparently, the final oxide product was obtained with the desired morphology and size.

In another report, Hou et al. described an interesting strategy to prepare double-shelled silica hollow particles (DSHPs) using single-hole hollow particles (SHHPs) of PS as the templates (Fig. 14). ${ }^{54}$ The entire preparation procedure could be divided into three steps. Initially, SHHPs were prepared by seed emulsion polymerization. Furthermore, via the Stöber method, both the inner and outer surfaces of SHHPs were coated with
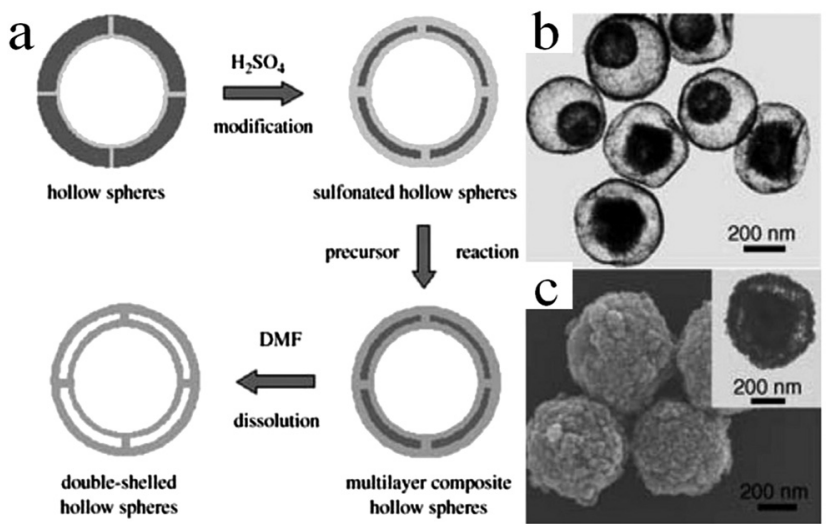

Fig. 13 (a) Schematic illustration of the synthesis of double-shelled inorganic hollow spheres. (b) TEM images of double-shelled $\mathrm{TiO}_{2}$ hollow spheres and (c) SEM and TEM images (inset) of $\mathrm{Fe}_{3} \mathrm{O}_{4}$. Reprinted from ref. 53 with kind permission.

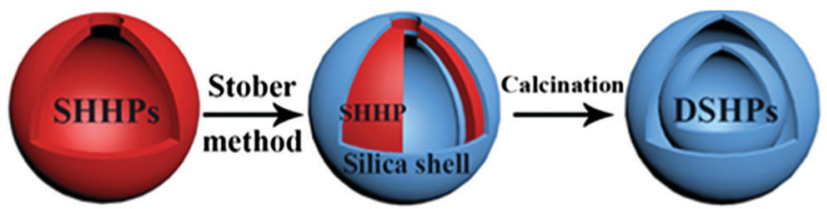

Fig. 14 Schematic of the preparation process of DSHPs. Reprinted from ref. 54 with kind permission.

silica shells. Finally, the intermediate SHHP layer was removed by calcination, thereby forming DSHPs. In addition, other double-shelled hollow microspheres such as $\mathrm{TiO}_{2}, \mathrm{Fe}_{3} \mathrm{O}_{4}$, $\mathrm{BaTiO}_{3}$, and conducting polymer (polypyrrole and polyaniline) were also successfully prepared by making use of sulfonated PS hollow spheres as the templates. ${ }^{55-59}$ It should be noted that the PS template method seems to be a little tedious and dangerous since concentrated $\mathrm{H}_{2} \mathrm{SO}_{4}$ is often utilized to treat PS colloidal microspheres.

Resorcinol-formaldehyde (RF) resins are another important polymer that can serve as a template for the synthesis of various functional materials. Similar to a carbonaceous template, RF resins can often siphon metallic ions and then transform into oxides with multishelled hollow structures when the operating conditions are precisely controlled. Li et al. used a RF resin microsphere as a sacrificial template for preparing a series of triple-shelled $\mathrm{Ni}-\mathrm{Co}-\mathrm{O}$ hollow microspheres with a delicate ratio of Ni/Co (Fig. 15a). ${ }^{60,61}$ The RF resin microspheres were obtained from 2,4-dihydroxybenzoic acid reacted with formaldehyde under hydrothermal conditions. Owing to the residence of numerous $-\mathrm{COOH}$ and $-\mathrm{OH}$ groups in the precursors, $\mathrm{RF}$ could facilitate the adsorption and penetration of metal ions

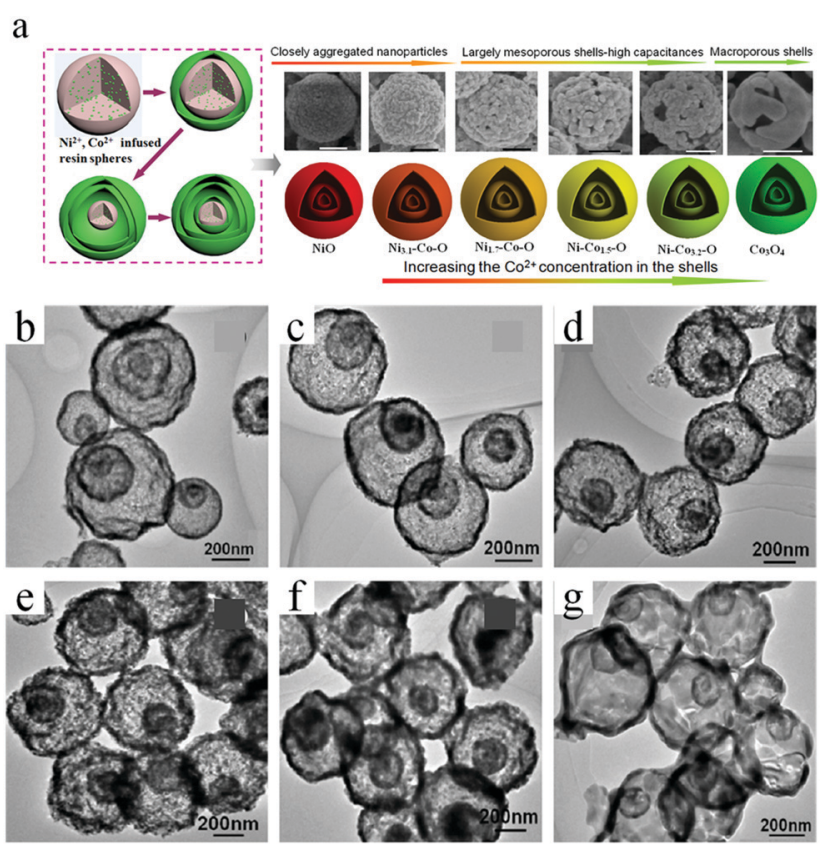

Fig. 15 (a) Schematic illustration of the preparation of triple-shelled $\mathrm{NiO}$, $\mathrm{CO}_{3} \mathrm{O}_{4}$, and their mixed oxide (Ni-Co-O) hollow spheres. (b-g) Typical TEM images of these samples. Reprinted from ref. 60 with kind permission. 
$\left(\mathrm{Ni}^{2+}\right.$ and/or $\left.\mathrm{Co}^{2+}\right)$. It should be noted that the adsorption procedure was performed in ethylene glycol but not in water. The reason might be related to the larger radii of hydrated $\mathrm{Ni}$ and Co ions, which are difficult to deeply diffuse into the RF template. After heating to $550{ }^{\circ} \mathrm{C}$ at the rate of $5{ }^{\circ} \mathrm{C} \mathrm{min}^{-1}$ and maintained for $4 \mathrm{~h}$ in an air atmosphere, the above $\mathrm{Ni}^{2+} / \mathrm{Co}^{2+}$. ion-infused RF resin could be converted into a triple-shelled oxide hollow microsphere with different Ni/Co ratios. Fig. 15b-g shows the TEM images of these Ni-Co-O samples, confirming that triple-shelled hollow structures were obtained. In addition, single- and double-shelled hollow structures may also be obtained by shortening the soaking time to $0.5 \mathrm{~h}$ and lowering the annealing rate to $2{ }^{\circ} \mathrm{C} \mathrm{min}^{-1}$. By varying the initial concentrations of $\mathrm{Ni}^{2+}$ and $\mathrm{Co}^{2+}$, the thickness of the triple-shelled hollow structures may be tuned. Recently, Zhu et al. prepared multishelled $\mathrm{CoFe}_{2} \mathrm{O}_{4}$ using a RF colloidal sphere as the hard template. $^{62}$

Polyacrylic acid (PAA) is an anionic polymer rich in carboxyls that can efficiently coordinate with metal ions. Therefore, PAA can also be used as a hard template to fabricate multishelled metal oxide hollow nanostructures. Qi et al. described the syntheses of $\mathrm{CoFe}_{2} \mathrm{O}_{4}$ (CFO) solid nanospheres (SNSs), hollow nanospheres (HNSs), and multishelled hollow nanospheres (MS-CFO-NHSs) using PAA- $\mathrm{NH}_{4}$ microspheres as the hard template. The synthesis mechanism is shown in Fig. $16 .^{63}$ Initially, $\mathrm{PAA}-\mathrm{NH}_{4}$ microspheres adsorb plenty of water containing $\mathrm{Fe}^{2+}$ and $\mathrm{Co}^{2+}$. Subsequently, $\mathrm{Fe}^{2+}$ and $\mathrm{Co}^{2+}$ are simultaneously hydrolyzed to form $\mathrm{Fe}(\mathrm{OH})_{2}$ and $\mathrm{Co}(\mathrm{OH})_{2}$, which are randomly dispersed in the PAA- $\mathrm{NH}_{4}$ microsphere. It is well known that $\mathrm{Fe}(\mathrm{OH})_{2}$ can be easily oxidized into $\mathrm{Fe}(\mathrm{OH})_{3}$; therefore, the CFO NCs precursor was generated. Finally, by precisely controlling the calcination process, CFO-SNSs, CFO-HNSs, and MS-CFO-HNSs were obtained.

Besides certain synthetic polymers, some natural polymers have also been good candidates for use as templates for preparing various functional materials with desirable structures. Alginate, a natural polysaccharide rich in hydroxyl groups, also
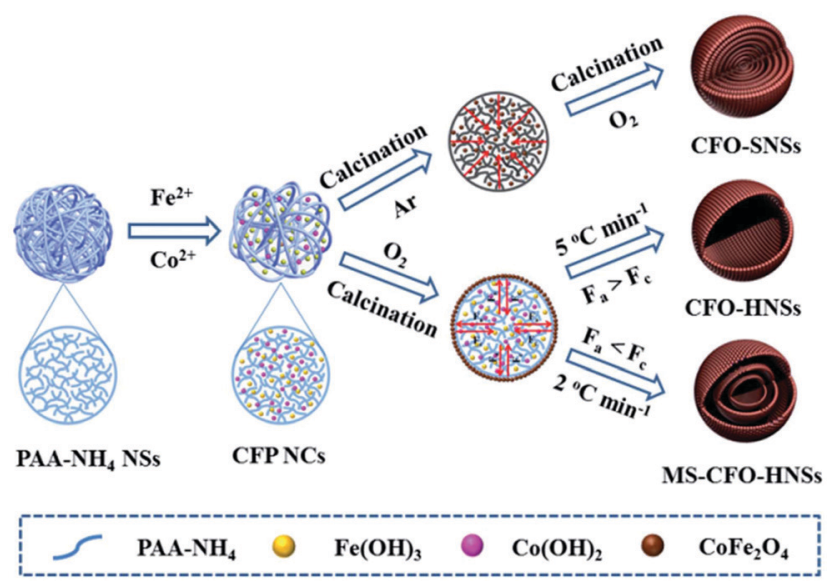

Fig. 16 Schematic illustrations of the formation processes of CFO-SNSs, CFO-HNSs, and MS-CFO-HNSs using PAA-NH $\mathrm{N}_{4}$ as the hard template. Reproduced from ref. 63 with permission.

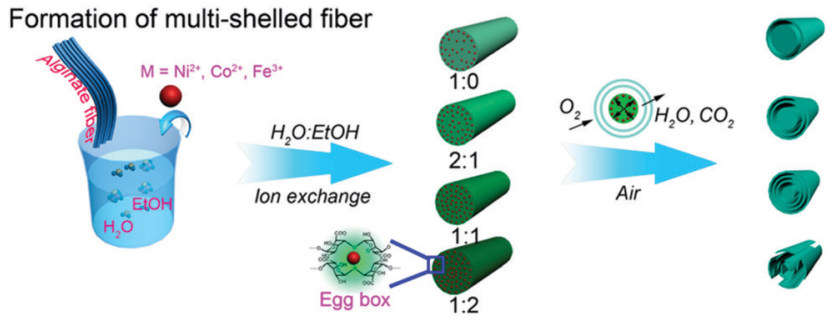

Fig. 17 Schematic illustration of the preparation of multishelled transition metal (Ni, Co, and $\mathrm{Fe}$ ) oxide hollow fibers using alginate as the template. Reproduced from ref. 64 with kind permission.

exhibits good affinity toward metal ions. It is expected that alginates with particular morphologies could direct the formation of transition metal oxides with multishelled hollow structures. Sun et al. developed a generic strategy to fabricate a class of multishelled hollow transition metal oxide nanofibers, such as $\mathrm{NiO}, \mathrm{Co}_{3} \mathrm{O}_{4}$, and $\mathrm{Fe}_{2} \mathrm{O}_{3}$, based on immobilizing metal ions into the alginate fibers followed by annealing in air, as shown in Fig. 17. Similar to carbonaceous and other polymer hard templates, the gradient distribution of transition metal ions is crucial for the formation of multishelled structures. Here, the ratio of ethanol to water in the solvent plays a significant role in the distribution of metal ions in the alginate template. The more ethanol in the solvent, the lower is the surface tension and higher is the wettability, which leads to deeper and higher adsorption of metal ions, yielding metal-alginate fibers. Upon performing a controlled annealing treatment, the transition metal-alginate fibers could be converted into multishelled hollow fibers. ${ }^{64}$

In addition to the abovementioned polymers, other polymers, such as polyacrylamide, ${ }^{65}$ PVP gel microspheres, ${ }^{66}$ etc., have also been used as hard templates for preparing multishelled hollow nanostructures. For many polymer resins as hard templates, there are many functional groups on their surfaces; therefore, no surface modification or activation steps are required in most cases. This considerably simplifies the processing steps and therefore consumes less time as compared to silica or carbonaceous template methods. On the basis of the properties of the polymer resin utilized, either specific solvent dissolution or calcination treatment could be employed for the removal of the polymer resin template. It should be noted that many polymer resins can get easily swollen in the liquid environment and expand at a relative high temperature; therefore, the reaction medium and temperature required to synthesize multishelled hollow materials using a polymer resin as the sacrificial template should be appropriately selected.

2.1.4 Metal-oxide-based hard templates. Pang et al. first reported the preparation of multishelled conducting polymer hollow microspheres, such as poly(3,4-ethylenedioxythiophene) and polypyrrole, with the use of $\mathrm{Fe}_{3} \mathrm{O}_{4}$ hollow spheres as the hard template. ${ }^{67}$ In their work, $p$-toluenesulfonic acid ( $p$-TSA) was utilized as the acid for the slow etching of $\mathrm{Fe}_{3} \mathrm{O}_{4}$ hollow spheres. In this way, the penetration of monomers into the inner surface of the template shell was effectively promoted. The detailed formation mechanism is shown in Fig. 18a. Firstly, the monomers were adsorbed on the outer surface of $\mathrm{Fe}_{3} \mathrm{O}_{4}$ 

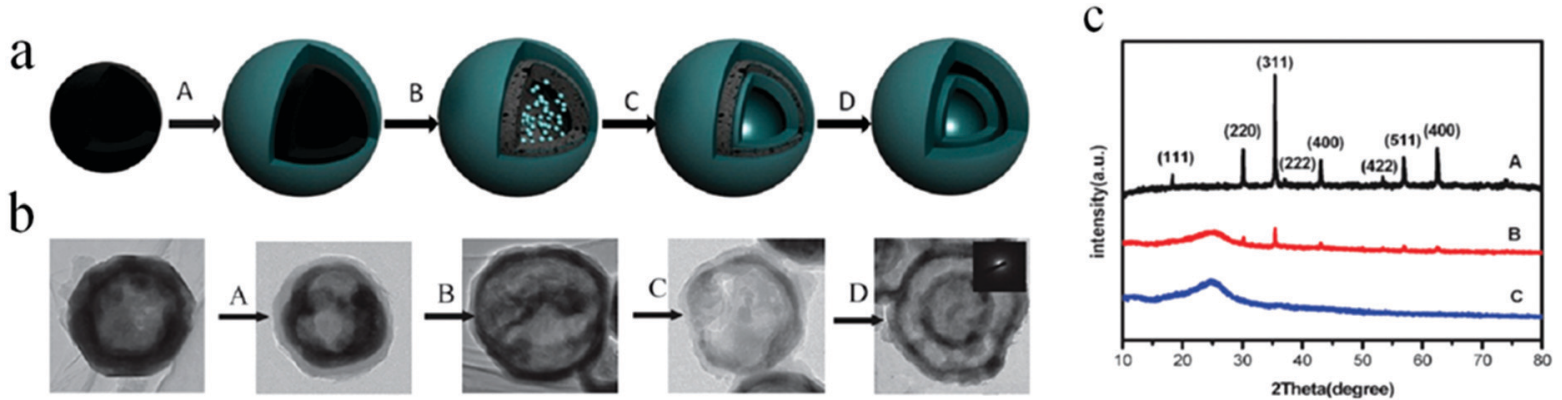

Fig. 18 (a) Proposed formation mechanism of double-shelled conducting polymer hollow microspheres using hollow $\mathrm{Fe}_{3} \mathrm{O}_{4}$ spheres as the template; (A) coating of a conducting polymer on the outer surface of hollow $\mathrm{Fe}_{3} \mathrm{O}_{4}$ spheres; (B) penetration of the monomer into the inner surface of the hollow $\mathrm{Fe}_{3} \mathrm{O}_{4}$ spheres; (C) in situ polymerization of the monomer on the inner surface of the hollow $\mathrm{Fe}_{3} \mathrm{O}_{4}$ spheres; (D) removal of the hollow Fe $\mathrm{O}_{4}$ spheres via slow etching using $p$-TSA. (b) TEM images of the product at each step. (c) XRD patterns of the product at different reaction times at $40{ }^{\circ} \mathrm{C}$ : (A) $0 \mathrm{~h}$; (B) $12 \mathrm{~h}$; and (C) 24 h. Reproduced from ref. 67 with kind permission.

hollow spheres and polymerized to form a conducting polymer (process A). Secondly, the monomer penetrated into the inner shell via the pores and voids generated by $p$-TSA through slow etching (process B), followed by in situ polymerization. Therefore, the inner surface of the $\mathrm{Fe}_{3} \mathrm{O}_{4}$ hollow spheres was also coated by a conducting polymer (process $\mathrm{C}$ ). Finally, owing to the existence of $p$-TSA, $\mathrm{Fe}_{3} \mathrm{O}_{4}$ hollow spheres could be completely etched gradually by reaction-temperature-programmed treatment (process D). The TEM images shown in Fig. 18b confirm the structural evolution, which is in good agreement with the abovementioned mechanism. In particular, it is evident that the selected area electron diffraction (SAED) pattern of the inner shell of the final product showed no obvious diffraction spots, indicating that the $\mathrm{Fe}_{3} \mathrm{O}_{4}$ hollow spheres were completely removed. Further, the XRD patterns of the time-dependent products are shown in Fig. 18c; notably, the intensities of the sharp peaks (curve A) indexed to the crystalline $\mathrm{Fe}_{3} \mathrm{O}_{4}$ phase became weak (curve B) after $12 \mathrm{~h}$, which ultimately disappeared after $24 \mathrm{~h}$ (curve $\mathrm{C}$ ), further confirming the proposed mechanism. In another study, Niu et al. prepared double-shelled polypyrrole hollow spheres using hollow $\mathrm{Fe}_{3} \mathrm{O}_{4}$ spheres as the sacrificial template via a two-step strategy, prefilling the pyrrole monomer on both the outer and inner surfaces followed by the in situ polymerization of pyrrole with $\mathrm{HCl}$ etching, yielding $\mathrm{Fe}^{3+}$ ions. ${ }^{68}$ By a polymer coatingcarbonization-post-doping treatment process using porous $\mathrm{Fe}_{3} \mathrm{O}_{4}$ hollow spheres as the templates, Qian and co-workers prepared pristine and double-shelled N-doped carbon hollow spheres. ${ }^{69}$ Employing hollow $\mathrm{TiO}_{2}$ spheres as the hard templates and dopamine (DA) as the N-containing carbon precursor, $\mathrm{N}$-doped double-shelled hollow carbon spheres were also obtained. ${ }^{70}$

2.1.5 Other hard templates. In addition to the hard templates discussed above, some other hard template candidates have also been researched, such as inorganic salts, elemental sulfur, ${ }^{71}$ and so on. Meanwhile, it should be noted that several hard templates can possibly be combined together for constructing multishelled hollow nanostructures with a complex composition in the future. Such hard templates have been specifically studied, as provided in the literature.
Apparently, the hard template method to synthesize multishelled hollow nanostructures exhibits several advantages. It is a versatile tool to prepare multishelled hollow structures with desirable morphology and show advantages in controlling the size and the diameter/cavity of the final product when compared with those obtainable via other techniques. Moreover, the final products obtained by the hard template strategy are expected to appear highly uniform with regard to particle size and morphology. The hard template method has at least three disadvantages. Firstly, presynthesized hard templates are necessary in most cases. The limited availability of raw materials, storage, and template transformation might be another bottleneck owing to the inherent properties of the template. Secondly, several other procedures, such as the coating of targeted materials or precursors on the surface of the template, as well as the ultimate removal of the template via etching or calcination, are often involved that may complicate the preparation process; posttreatment may destroy the final product to a certain extent. Thirdly, on the basis of tedious synthesis procedures, it is impractical to produce multishelled hollow nanostructures at a large scale with the hard template method, particularly for nanostructures with a higher number of shells (over 5 shells).

\subsection{Soft template approach}

In addition to the hard template method, soft template methods have also been versatile tools for the fabrication of multishelled hollow nanostructures. Generally, the soft template approach used to fabricate multishelled hollow nanostructures involves the self-assembly of surfactants/cosurfactants that can yield relatively flexible structures such as emulsion droplets, micelles, gas bubbles, and vesicles, etc. On the basis of the interactions between the soft template/solvent and precursors, the shell of the target materials may be initially produced, which could subsequently grow within the interfacial region during template removal. Until now, the soft template method has been widely applied in transcriptase synthesis with diverse hollow structures.

2.2.1 Single surfactant as soft templates. It is a well-known fact that amphiphilic molecules, such as surfactants and block copolymers, can form vesicles or micelles with different structures, 


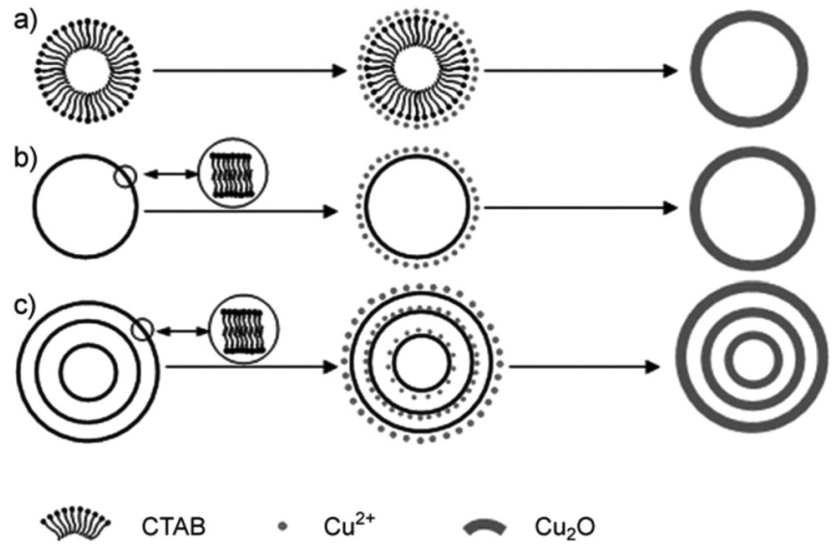

Fig. 19 Schematic of the formation mechanism of single-shelled and multishelled $\mathrm{Cu}_{2} \mathrm{O}$ hollow spheres using $\mathrm{CTAB}$-assembled vesicles as the soft template. Reprinted from ref. 75 with kind permission.

which are widely employed as a soft template (also called a structure-directing agent) to synthesize various functional materials. ${ }^{72-74}$ Inspired by the preparation of single-shelled hollow nanostructures using micelles and vesicles as the soft template (Fig. 19a and b), Xu and co-workers proposed that growing the target materials on all the layers of multilamellar vesicles may lead to multishelled hollow nanostructures, as shown in Fig. 19c. ${ }^{75}$ By adjusting the concentration of cetyltrimethylammonium bromide (CTAB), the number of shells can be controlled (Fig. 20). In particular, the doubleshelled spherical structures are dominant for a CTAB concentration of $0.13 \mathrm{M}$; interestingly, triple-shelled structures were also observed. When the CTAB concentration increased to $0.15 \mathrm{M}$, triple- and quadruple-shelled hollow spheres were observed. This might be the disadvantage of this system. ${ }^{75}$ In another case, Wang et al. also prepared $\mathrm{Cu}_{2} \mathrm{O}$ hollow submicron spheres with single and double shells using CTAB vesicles as the soft template and ascorbic acid as the reducing agent. $^{76}$

Teng and co-workers extended CTAB as a soft template to fabricate multishelled periodic mesoporous organosilica (PMO) hollow spheres (Fig. 21). ${ }^{77}$ The entire process could be divided into four steps. First, TEOS and 1,4-bis(triethoxysilyl)benzene (BTSE) were transformed into mesostructured ethane-bridged organosilica spheres via a modified Stöber method using a CTAB-surfactant-directed sol-gel process. Subsequently, the

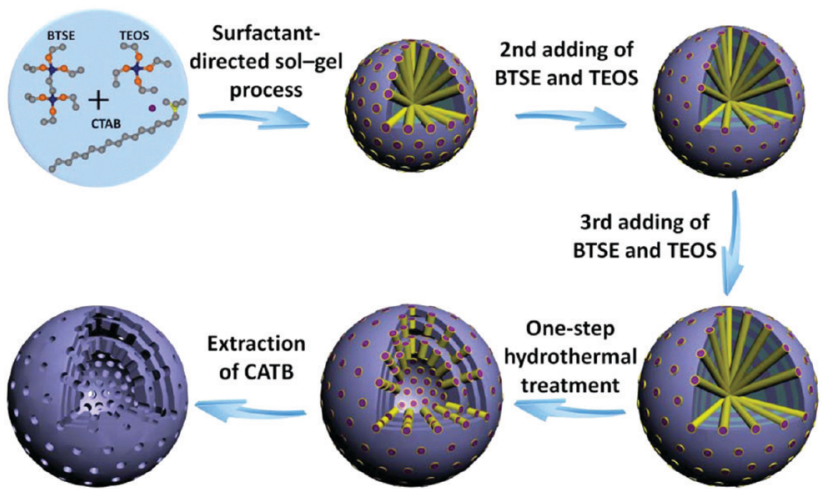

Fig. 21 Schematic illustration of the synthesis procedure and successive growth process of triple-shelled PMO hollow spheres. Reprinted from ref. 77 with kind permission.

repeated addition of TEOS and BTSE resulted in the formation of multilayered organosilica/CTAB spherical composites with enhanced sizes. The number of shells of the ultimately obtained multishelled PMO hollow spheres was dependent on the addition times of the TEOS and BTSE precursors. Thereafter, hydrothermal treatment was carried out to transform the subsequently grown solid organosilica spheres into hollow structures. Finally, CTAB surfactants were extracted by acidic ethanol, yielding multishelled PMO hollow spheres. By precisely controlling the synthesis conditions, such as CTAB concentration, amount of organosilica cores, and reaction temperature, the overall diameter, intershell space, and shell thickness of the finally obtained PMO hollow spheres could be easily tuned.

Yang et al. developed a self-assembly procedure to prepare multishelled ZnO hollow spheres by using a triblock copolymer Pluronic P123 as the soft template. ${ }^{78}$ The preparation mechanism included the following steps, as shown in Fig. 22. Initially, P123 and a short-chain alcohol were self-assembled into spherical reverse micelles, which may provide space to accommodate inorganic oligomers. Subsequently, a solvothermal treatment was conducted to improve the organization, induce complete condensation, and crystallization. At this stage, by the Ostwald ripening of various intercrystallite spaces within these structures, a void space was created. When pristine noncompact aggregates were transformed into a smaller encapsulated aggregate, double-shelled $\mathrm{ZnO}$ hollow spheres were generated.
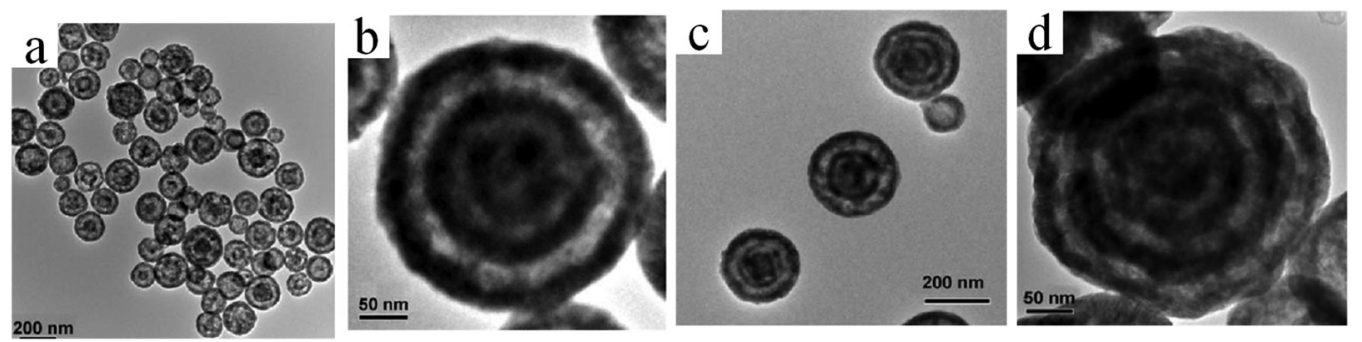

Fig. 20 TEM images of (a) double-shelled, (b and c) triple-shelled, and (d) quadruple-shelled $\mathrm{CuO}_{2}$ hollow spheres for different CTAB concentrations: ( $a$ and b) 0.13 and (c and d) $0.15 \mathrm{M}$. Reprinted from ref. 75 with kind permission. 


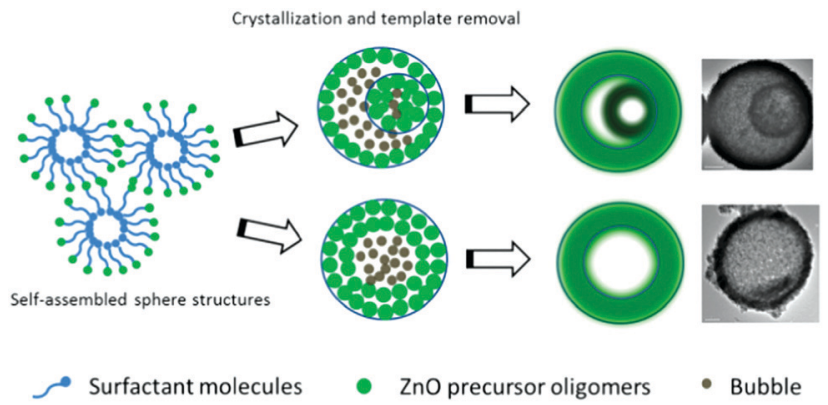

Fig. 22 Synthesis mechanism of shelled ZnO hollow spheres using P123 as the soft template. Reprinted from ref. 78 with kind permission.

Otherwise, if the $\mathrm{ZnO}$ aggregates were compact, $\mathrm{ZnO}$ hollow spheres with single-shelled structures were produced. Finally, when washed with water and ethanol, the surfactant template was removed, resulting in the formation of well-crystallized shelled ZnO hollow spheres. Taking full advantage of the characteristics of the self-assembly of micelles by surfactants, Zhang et al. reported the synthesis of organosiliceous multilamellar vesicles using the commercially available triblock copolymer Pluronic P85 as a single template and 1,2-bis(triethoxysilyl)ethane as the organosilica precursor. The number of shells of organosilica could be precisely controlled (within 1 to 7 ) by adjusting the $\mathrm{pH}$ of the solution from 4.8 to $5.2 .{ }^{79}$

$\mathrm{Xu}$ et al. described a soft template method to prepare double-shelled hollow mesoporous carbon nanospheres using (polystyrene-b-polyacrylic acid) (PS- $b$-PAA) micelles as the soft template. The entire formation process is shown as Fig. 23, which involves several stages.$^{80}$ Firstly, on the basis of electronic interactions, positively charged DA molecules and negatively charged PS- $b$-PAA blocks assembled into large micelles, i.e., PS- $b$-PAA/DA, in which multiple reverse small PAA- $b$-PS/DA micelles were present. Subsequently, DA molecules and TEOS would undergo self-polymerization and hydrolysis/condensation in the PS- $b$-PAA/DA composite micelles with the assistance of $\mathrm{NH}_{3} \cdot \mathrm{H}_{2} \mathrm{O}$, forming polydopamine (PDA) and $\mathrm{SiO}_{2}$, respectively, on the surface of the micelles. As the reaction proceeded, the residual

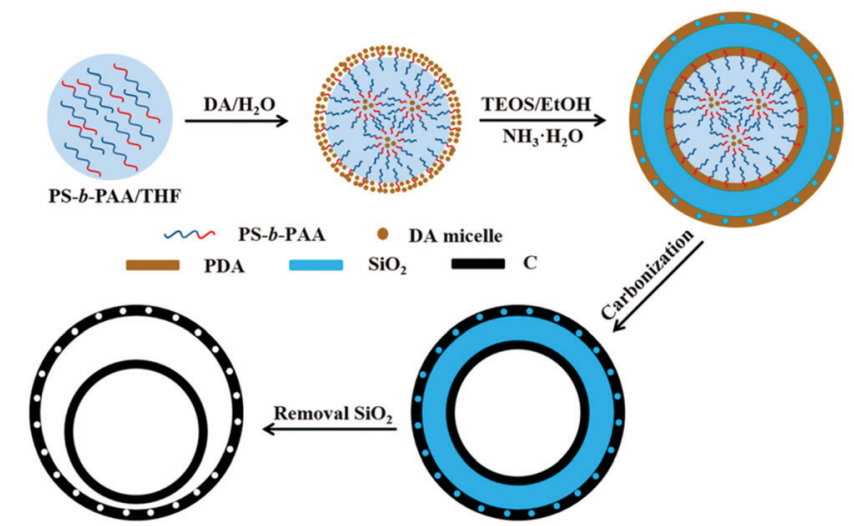

Fig. 23 Schematic illustration of the formation process of double-shelled hollow mesoporous carbon nanospheres using PS- $b$-PAA micelles as the soft template. Reproduced from ref. 80 with permission.
DA continued to form PDA on the outermost layer. Thereafter, the PDA was converted into N-doped carbon via carbonization at higher temperatures, generating three-layered $\mathrm{C} / \mathrm{SiO}_{2} / \mathrm{C}$ nanostructures. Finally, the intermediate layer of $\mathrm{SiO}_{2}$ was removed by $\mathrm{NaOH}$ etching, which resulted in the formation of double-shelled hollow nitrogen-doped carbon mesoporous nanospheres. It should be noted that the dosage ratio of TEOS/ DA had a significant influence on the formation of the final product owing to the competitive relationship between the DA self-polymerization and TEOS hydrolysis/condensation under alkaline conditions. These research results demonstrated that only an intermediate dosage of TEOS could lead to the formation of double-shelled carbon hollow structures as the main product. At lower dosages of TEOS, DA self-polymerization would assume the dominant role, while TEOS oligomers might act as a template to generate mesopores in the carbon spheres. At higher dosages of TEOS, silica networks may dominate in the $\mathrm{SiO}_{2} / \mathrm{C}$ intermediate; as a result, an inconsecutive outer carbon shell could be formed, while the outer carbon shell may disappear during the subsequent silica removal process. If the dosage of TEOS is extreme high, only certain carbon fragments can be obtained.

2.2.2 Dual/multiple surfactants as soft templates. To produce multishelled hollow nanostructures with mesoporous silica shells, Liu and co-workers proposed a vesicle template route, which was derived from the self-assembly of surfactants $\left[\mathrm{C}_{3} \mathrm{~F}_{7} \mathrm{O}\left(\mathrm{CFCF}_{3} \mathrm{CF}_{2} \mathrm{O}\right)_{2} \mathrm{CFCF}_{3} \mathrm{CONH}\left(\mathrm{CH}_{2}\right)_{3} \mathrm{~N}^{+}\left(\mathrm{C}_{2} \mathrm{H}_{5}\right)_{2} \mathrm{CH}_{3} \mathrm{I}^{-}\right](\mathrm{FC} 4)$ and $\mathrm{EO}_{106} \mathrm{PO}_{70} \mathrm{EO}_{106}$ (F127), as shown in Fig. 24a. ${ }^{81}$ The mixing of FC4 and F127 in a water-ethanol solution spontaneously generated vesicles, which was confirmed by a light-scattering study; the acidity of the solution was adjusted by an ammonia solution. Subsequently, the growth of silica would occur on the addition of TEOS by the interaction of silane precursors and the surface of FC4 vesicles. It was also found that the diameter, number of shells, and shell thickness of multishelled mesoporous silica nanospheres could be easily tuned by varying the experimental parameters. Fig. 24b-g show the typical SEM and TEM images of multishelled mesoporous silica hollow nanospheres obtained with different molar ratios of FC4/F127. Obviously, at a low FC4/F127 molar ratio, the size distributions are narrow (Fig. 24b and c), while it is relatively broader at a high FC4/F127 molar ratio (Fig. 24d). In particular, according to the observation results from the TEM images, the number of shells and thickness also appear to be different with a change in the FC4/F127 molar ratio. When the FC4/F127 molar ratio is 24 and 48, the average diameters of the nanospheres are less than $150 \mathrm{~nm}$, and the number of shells was $\sim 3-4$ with a thickness of about $10 \mathrm{~nm}$ (Fig. 24e and f). With regard to a FC4/F127 molar ratio of 72, the average diameter of the nanospheres ranged within $\sim 100-400 \mathrm{~nm}$, and the number of shells decreased to $\sim 1-3$ (Fig. 24g). Such a result might be attributed to the transformation of uniform multilamellar vesicles to single lamellar vesicles upon the addition of FC4. The effect of $\mathrm{pH}$ of the system was also investigated to confirm the vesicle template mechanism. Other vesicle templates of cosurfactant combinations, such as CTAB and dodecyl benzene sulfonate (SDBS), ${ }^{82} \mathrm{P} 123$ and 1,3,5-triisopropylbenzene (TIPB), ${ }^{83} \mathrm{FC} 4$ and 

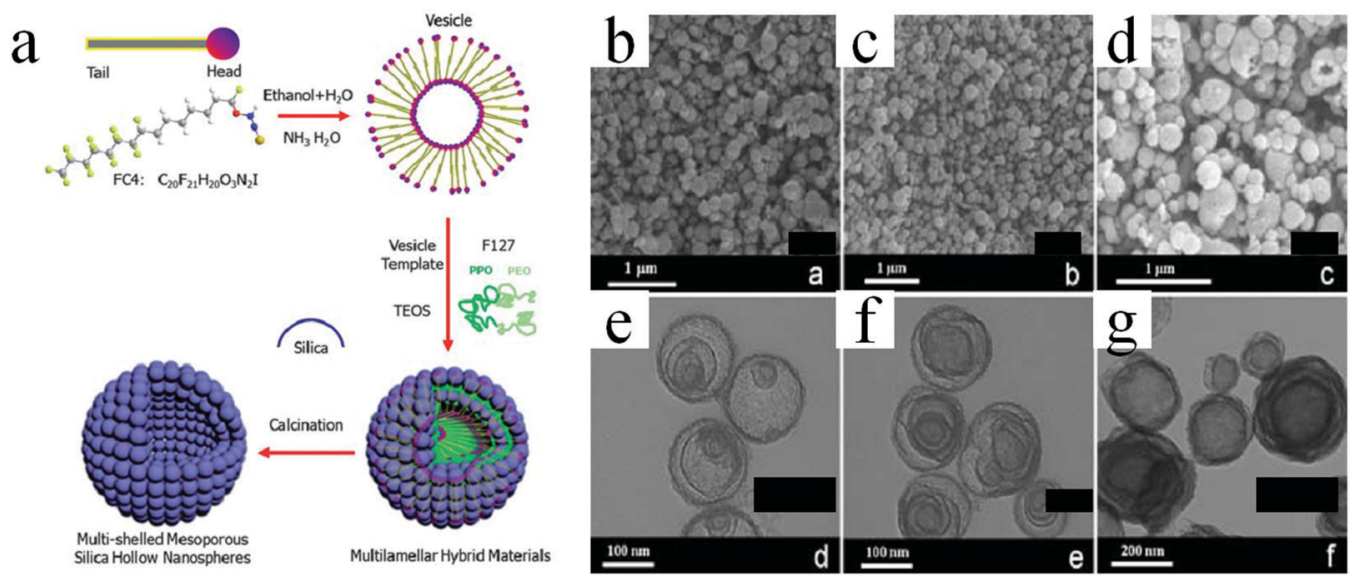

Fig. 24 (a) Schematics of the synthesis procedures of multishelled mesoporous silica hollow nanospheres. (b-d) SEM and (e-g) TEM images of multishelled mesoporous silica hollow nanospheres obtained for different molar ratios of FC4 and F127. (b and e) FC4/F127 = 24, (c and f) FC4/F127 = 48, and $(\mathrm{d}$ and $\mathrm{g}) \mathrm{FC} 4 / \mathrm{F} 127=72$. Reprinted from ref. 81 with kind permission.

$\mathrm{CTAB},{ }^{84}$ didodecyldimethylammonium bromide (DDAB)/CTAB, ${ }^{85}$ CTAB and sodium salicylate, ${ }^{86}$ and perfluorooctanoic acid (PFOA) and $\mathrm{CTAB},{ }^{87}$ were applied to synthesize multishelled mesoporous silica nanostructures.

Huang et al. developed a facile and sustainable strategy by using compressed $\mathrm{CO}_{2}$ to prepare multishelled hollow PMO nanospheres, in which the soft template is composed of the triblock copolymer Pluronic P123, CTAB, and sodium dodecyl sulfate (SDS). ${ }^{88}$ The formation mechanism of multishelled hollow PMOs comprises several steps, as shown in Fig. 25a. Similar to other soft template processes, the P123 + CTAB + SDS surfactants were initially self-assembled into vesicles in a solution. The penetration of $\mathrm{CO}_{2}$ into the hydrocarbon chain region of the surfactant molecules may cause volume expansion and increase in the blending energy when compressed $\mathrm{CO}_{2}$ was passed into the solution. Consequently, the mixed surfactants of CTAB and SDS formed multilamellar vesicles. Thereafter, the precursor, i.e., BTSE, underwent a hydrolysis reaction to form organosilica/vesicle composites. After the extraction of the surfactants by ethanol-HCl $(100: 3, \mathrm{v} / \mathrm{v})$, multishelled PMO hollow nanospheres were obtained. The number of shells/ thickness and the overall diameter of the final products could be easily modulated by precisely adjusting the $\mathrm{CO}_{2}$ pressure. Fig. 25b-f show the typical TEM images of these products with different numbers of shells by tuning the $\mathrm{CO}_{2}$ pressure.

As discussed above, soft template approaches possess the advantages of template removal since the process is expected to consume less energy than those by hard template approaches.
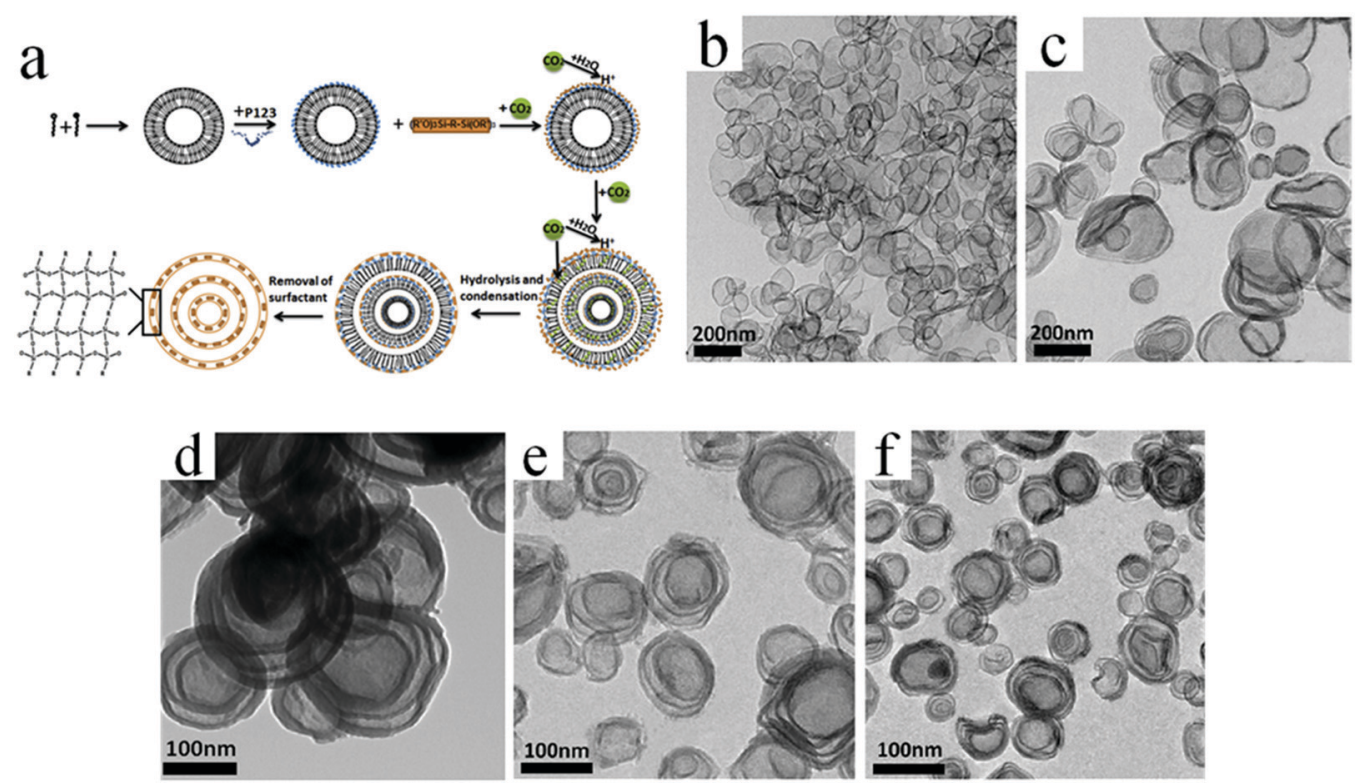

Fig. 25 (a) Formation mechanism of multishelled hollow PMO nanospheres using P123, CTAB, and SDS as a dual template with the assistance of compressed $\mathrm{CO}_{2}$. (b-f) TEM images of multishelled PMO hollow nanospheres obtained at $\mathrm{CO}_{2}$ pressures of 3.90 (b), 4.40 (c), 4.90 (d), 5.60 (e), and 5.90 (f) $\mathrm{MPa}$. Reproduced from ref. 88 with kind permission. 
Although tremendous progress has been made in the soft template synthesis of multishelled hollow nanostructures, the utilization of soft templates for synthesizing multishelled hollow nanostructures is not trivial since the soft templates themselves, including micelles, emulsion droplets, vesicles, gas bubbles, and other organized molecular assemblies, are usually not very stable under varying environments. Any marginal changes in $\mathrm{pH}$, temperature, solvent, ionic strength, or any additives may destroy the structure of the soft template, resulting in failure in the preparation of the final products. As a consequence, the soft template method provides less control over particle uniformity. Meanwhile, it is difficult to completely remove the soft template, and the residual organic molecular assembly might affect the purity of the final products. Furthermore, in light of being environmentally benign, the soft template approach consumes large amounts of organic solvents and reagents, which may be harmful to the environment as well as living organisms. In addition, different from the hard template approaches, it is difficult to extend the soft template approach from one system to another.

\subsection{Self-template approaches}

Self-template methods without requiring an additional template material are more preferable in practical applications for creating hollow materials. Different from the hard-/softtemplate-based approaches, the templates utilized in the selftemplate approaches may play multiple roles in the synthesis of multishelled hollow materials. ${ }^{89,90}$ The first one is that the template might comprise the final multishelled hollow architecture. The second one is that the template may act as a precursor to generate multishelled hollow materials. In addition, the template might also react during the formation of multishelled hollow nanomaterials, thereby generating a void structure in the final products. Until now, several materials have been demonstrated to be promising self-template candidates for the fabrication of multishelled hollow nanostructures. In this section, we will discuss certain examples and related mechanisms.

2.3.1 CPs or MOFs as self-templates. A case in point in which a self-template could be used to construct multishelled hollow structures might be CPs or MOFs, organometallic complexes comprising metal ions or metal clusters and organic ligands. MOFs have garnered extensive research interest owing to their promising applications in diverse fields such as catalysis, gas separation, and biomedicine. Various complex hollow nanostructures can be obtained by using unique reactivity and thermal properties of CPs and MOFs. ${ }^{91-93}$ Liu et al. developed a step-by-step crystal growth strategy followed by post-etching processes to prepare multishelled hollow $\mathrm{Cr}(\mathrm{III})$ terephthalate MOFs (MIL-101) with single-crystalline shells (Fig. 26a). ${ }^{94}$ In particular, octahedron-shaped MIL-101 crystals (average size: $\sim 200 \mathrm{~nm}$ ) were prepared by the reaction of $\mathrm{Cr}^{3+}$ and terephthalic acid under hydrothermal conditions. It is well known that MIL-101 possesses cavities of about $3.4 \mathrm{~nm}$ in its crystal structure, enabling mass transport via pentagonal and hexagonal windows. ${ }^{95,96}$ Further, it has been demonstrated that the inner crystallites were less stable than the outer crystallites. As a consequence, once treated with acetic acid, the inner crystallites would be preferentially etched, resulting in hollow voids inside the MIL-101 crystals. By repeating the in situ growth of MIL-101 before the final etching operation, more inner crystallite layers can be obtained. Taking advantage of the different stabilities of these inner and relative outer crystallites, the etching operation could lead to multishelled hollow MIL-101 architectures, such as single-shelled hollow MIL-101 (SSHM), double-shelled hollow MIL-101 (DSHM), and tripleshelled hollow MIL-101 (TSHM). Fig. 26b-e shows the typical TEM images and corresponding fast Fourier transform (FFT) patterns (insets, Fig. 26b-e) of these samples with controlled numbers of shells. It is apparent that each crystal appears with a spherical shape with hollow voids in the interior. It should be noted that both cavity size and shell thickness could be tuned by controlling the etching time.

Researchers have noted that MOFs are robust precursors in the preparation of various functional materials with different structures, particularly complex hollow structures. Cho et al. reported the synthesis of multishelled hybrid metal oxide hollow microspheres by using coordination polymer microspheres as the template and combining cation exchange followed
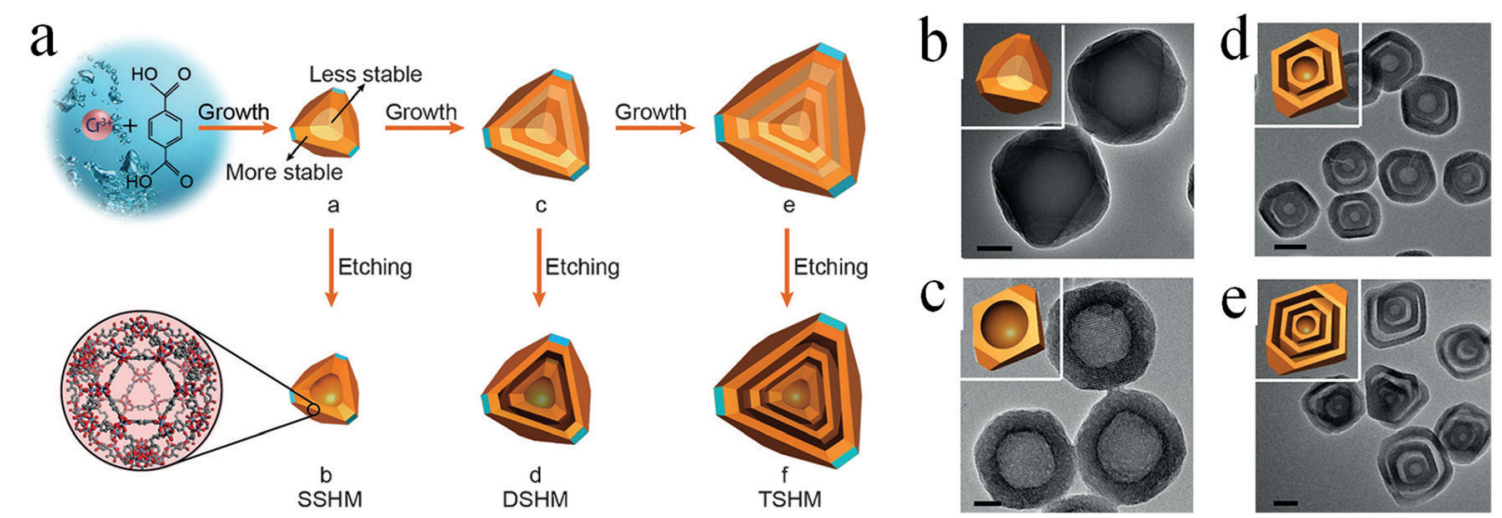

Fig. 26 (a) Schematic illustration of the preparation procedure of multishelled hollow MIL-101 by step-by-step and post-etching processes. TEM images of solid MIL-101 (b), SSHM (c), DSHM (d), and TSHM (e). Scale bars: (b and c) 50 nm; (d and f) $200 \mathrm{~nm}$. Insets in (b-e) show the corresponding FFT patterns. Reprinted from ref. 94 with kind permission. 


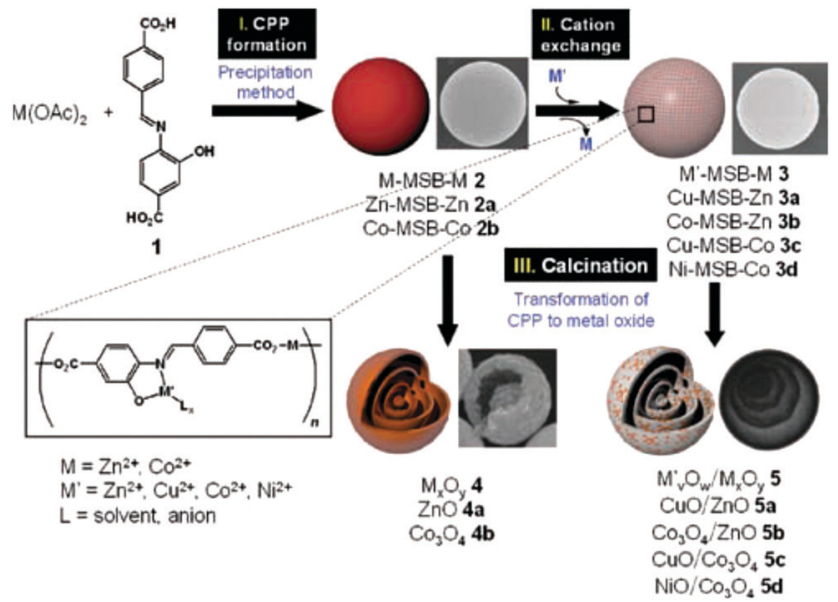

Fig. 27 Schematic illustration of the preparation of multishelled hybrid metal oxide hollow microspheres. Reproduced from ref. 97 with permission.

by controlled thermal treatment. ${ }^{97}$ The entire preparation process involves several procedures, as shown in Fig. 27. Firstly, solid spherical CPs containing $\mathrm{Zn}^{2+}$ or $\mathrm{Co}^{2+}$ were synthesized through a precipitation route. Subsequently, secondary metal ions (such as $\mathrm{Ni}^{2+}$ or $\mathrm{Cu}^{2+}$ ) were introduced via the ionic exchange process. Finally, calcination treatment was carried out to generate multishelled mixed metal oxide hollow microspheres. On eliminating the ionic exchange procedure, pure $\mathrm{ZnO}$ or $\mathrm{Co}_{3} \mathrm{O}_{4}$ multishelled hollow microspheres could be obtained. Initially, the outermost part of the solid spherical CPs was transformed into a ZnO shell when thermal treatment was applied. Thereafter, the internal CP component shrunk, accompanied by its decomposition into metal oxide species. Such a stepwise fabrication process of the metal oxide layers yielded the formation of multishelled metal oxide hollow microspheres.

Zhang et al. demonstrated an effective strategy to prepare multishelled ZnS-CdS rhombic dodecahedral cages (RDC) using zinc zeolitic imidazole framework ZIF-8 as the precursor. As shown in Fig. 28, the entire process includes three steps. Firstly, ZIF-8 RD was converted into yolk-shell RDC when etched with tannic acid. Secondly, the yolk-shell ZIF-8 RDC would react with thioacetamide as the sulfidation agent under solvothermal conditions to form multishelled ZnS RDC. Finally, multishelled ZnS-CdS RDC could be obtained by a hydrothermal cation exchange reaction. It should be noted that the composition of multishelled ZnS-CdS RDC could be effec- tively tuned by adjusting the cation exchange reaction time. Furthermore, the number of shells of the final $\mathrm{ZnS}-\mathrm{CdS}$ RDC was considerably dependent on the size of the ZIF RD precursor. ${ }^{98}$ By using similar procedures, Lou and co-workers undertook pioneering work in transforming CPS or MOFs into various multishelled hollow nanostructures, including metal oxides, ${ }^{99}$ metal oxyphosphides, ${ }^{100}$ and some complex metal oxides. ${ }^{101,102}$

2.3.2 Metal glycerates/glycolates as self-templates. Glycerol and ethylene glycol are small organic molecules rich in hydroxide groups in their molecule structures, possessing excellent binding ability to various metallic ions to form solid spheres of metal glycerates/glycorates. Taking advantage of the thermal property and reactivity, metal glycerate/glycolate can be converted into various complex hollow functional materials. ${ }^{103}$ For example, Zhang et al. designed double-shelled $\mathrm{ZnMn}_{2} \mathrm{O}_{4}$ hollow spheres via the thermal treatment of ZnMn glycolate hollow microspheres, which were prepared by a simple reflux method. ${ }^{104}$ The relocation of matter led to shell separation, which was driven by competition between the contraction and adhesion forces. Taking complete advantage of this concept, Li et al. extended this strategy toward the synthesis of various $\mathrm{MnCo}_{2} \mathrm{O}_{4}$ hollow spheres, including mesoporous spheres, single-shelled hollow spheres, yolk-shell spheres, double-shelled hollow spheres, and core-in-double-shell hollow spheres. ${ }^{105}$

Shen et al. reported the synthesis of $\mathrm{NiCo}_{2} \mathrm{~S}_{4}$ ball-in-ball (double-shelled) hollow spheres using NiCo glycerate as the precursor and thioacetamide (TAA) as the sulfur source, as shown in Fig. $29 .{ }^{106}$ Initially, TAA decomposed to generate sulfide $\left(\mathrm{S}^{2-}\right)$ under solvothermal conditions. $\mathrm{S}^{2-}$ ions easily reacted with metal ions on the surface of NiCo glycerate through anion exchange, forming core-shell-structured NiCo glycerate@ $\mathrm{NiCo}_{2} \mathrm{~S}_{4}$. As the reaction proceeded, a well-defined gap between the shell and NiCo glycerate core was observed owing to the low rate of inward diffusion of $\mathrm{S}^{2-}$ ions and fast rate of outward diffusion of metal cations. When the reaction proceeded to a certain degree, the outward diffusion of metal cations would become restricted; therefore, a new $\mathrm{NiCo}_{2} \mathrm{~S}_{4}$ shell might be formed on the inner surface of the remaining NiCo glycerate core, forming yolk-shell-structured intermediates. Finally, unique $\mathrm{NiCo}_{2} \mathrm{~S}_{4}$ ball-in-ball hollow spheres were obtained because of the competition of the anion exchange reaction. Recently, they extended this procedure to prepare $\mathrm{NiCO}_{2} \mathrm{~V}_{2} \mathrm{O}_{8}$ yolk-double-shelled spheres using NiCo glycerate as the precursor. ${ }^{107}$ Making full use of this concept, starting from

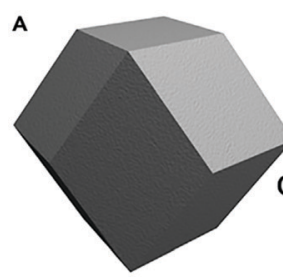

ZIF-8 RD

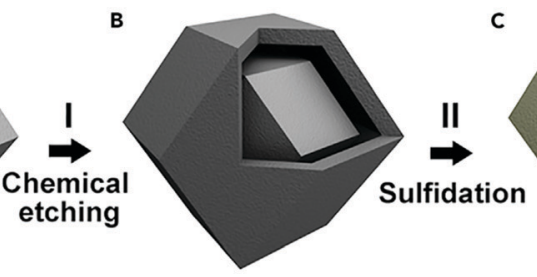

Yolk-shelled ZIF-8 RDC
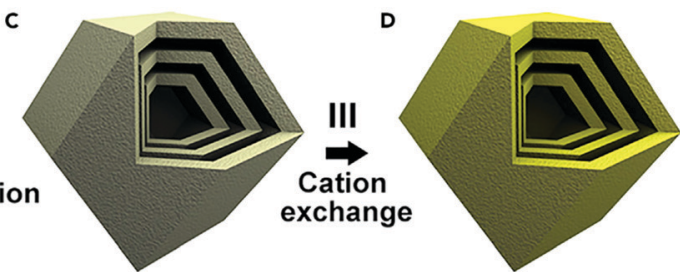

Multi-shelled ZnS RDC
Multi-shelled ZnS-CdS RDC

Fig. 28 Schematic illustration of the synthesis procedure for multishelled ZnS-CdS RDC. Reproduced from ref. 98 with kind permission. 


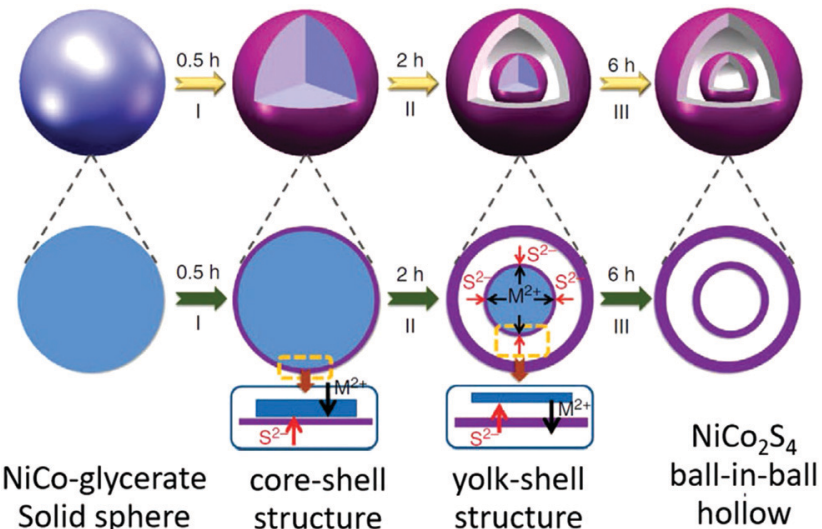

Fig. 29 Schematic illustration of the preparation of double-shelled $\mathrm{NiCO}_{2} \mathrm{~S}_{4}$ ball-in-ball hollow spheres via the sulfidation of NiCo glycerate solid spheres. Reprinted from ref. 39 with permission.

solid spheres of Mo glycerate, highly uniform Mo-PDA with triple-shelled hollow structures can be obtained through a facile solvothermal process. After thermal treatment, the triple-shelled Mo-PDA hollow spheres could be further converted into tripleshelled $\mathrm{MoO}_{2} / \mathrm{C}$ composite hollow spheres. ${ }^{108}$

2.3.3 Metal-inorganic precursors as self-templates. Besides MOFs and metal-containing polymers/compounds, some inorganic precursors such as oxides, hydroxides, and carbonates have also been employed as self-templates for the preparation of various hollow nanostructures. Guo et al. reported the synthesis of double-shelled CuS hollow nanocages using $\mathrm{Cu}_{2} \mathrm{O}$ nanocubes as the self-template, which acted as a precursor of the copper source to form double-shelled CuS hollow nanocages. ${ }^{109}$ A schematic of the synthesis procedure is shown in Fig. 30a. The main process involves sulfidation followed by controlled etching. The presynthesized $\mathrm{Cu}_{2} \mathrm{O}$ nanocubes were sulfided with $\mathrm{Na}_{2} \mathrm{~S}$ to form $\mathrm{Cu}_{2} \mathrm{O} @ \mathrm{CuS}$ core-shell structures (Fig. 30b). Subsequently, yolk-shell-structured $\mathrm{Cu}_{2} \mathrm{O} @ \mathrm{CuS}$ (Fig. 30c) would be obtained via the controlled partial etching of the $\mathrm{Cu}_{2} \mathrm{O}$ core with dilute $\mathrm{HCl}$. It should be noted that if the $\mathrm{Cu}_{2} \mathrm{O}$ core was completely etched, single-shelled CuS hollow nanocages could be obtained. For preparing the double-shelled structures, the yolk-shellstructured $\mathrm{Cu}_{2} \mathrm{O}$ @CuS could be further treated with $\mathrm{Na}_{2} \mathrm{~S}$, generating yolk-double-shell-structured $\mathrm{Cu}_{2} \mathrm{O} @ \mathrm{CuS} @ \mathrm{CuS}$ (Fig. 30d), followed by the complete removal of the residual $\mathrm{Cu}_{2} \mathrm{O}$ core through etching (Fig. 30e). Theoretically, the sulfidation and partial etching could be repeatedly carried out several times to produce additional number of shells in the final products. Unfortunately, the authors did not do further work.

$\mathrm{Wu}$ et al. reported the synthesis of multishelled $\mathrm{VOOH}$ hollow nanospheres by the thermal decomposition of $\mathrm{V}(\mathrm{OH})_{2} \mathrm{NH}_{2}$ by exercising precise control over the external reaction temperature. ${ }^{110}$ As shown in Fig. 31, the formation process may yield a solid sphere, core-shell structure, and multishelled hollow structure on the basis of the Kirkendall and Ostwald ripening effects. By adjusting the experimental parameters, the number of shells could be precisely controlled. In addition to oxides/hydroxides, metal carbonates were also reported to be versatile precursors to form multishelled hollow nanostructures. For example, Lin et al. prepared triple-shelled $\mathrm{Mn}_{2} \mathrm{O}_{3}$ hollow nanocubes using a directly programmable annealing treatment with $\mathrm{MnCO}_{3}$. Expectedly, the interaction between the contraction force from the decomposition of $\mathrm{MnCO}_{3}$ and the adhesion force from the formation of $\mathrm{Mn}_{2} \mathrm{O}_{3}$ resulted in the formation of a hierarchical structure. ${ }^{111}$

\subsection{Template-free approach}

Despite the considerable progress made in template approaches that have been used to fabricate multishelled hollow nanostructures, due to the complex nature and composition of the template, template preparation always remains challenging for researchers. Therefore, template-free approaches for preparing multishelled
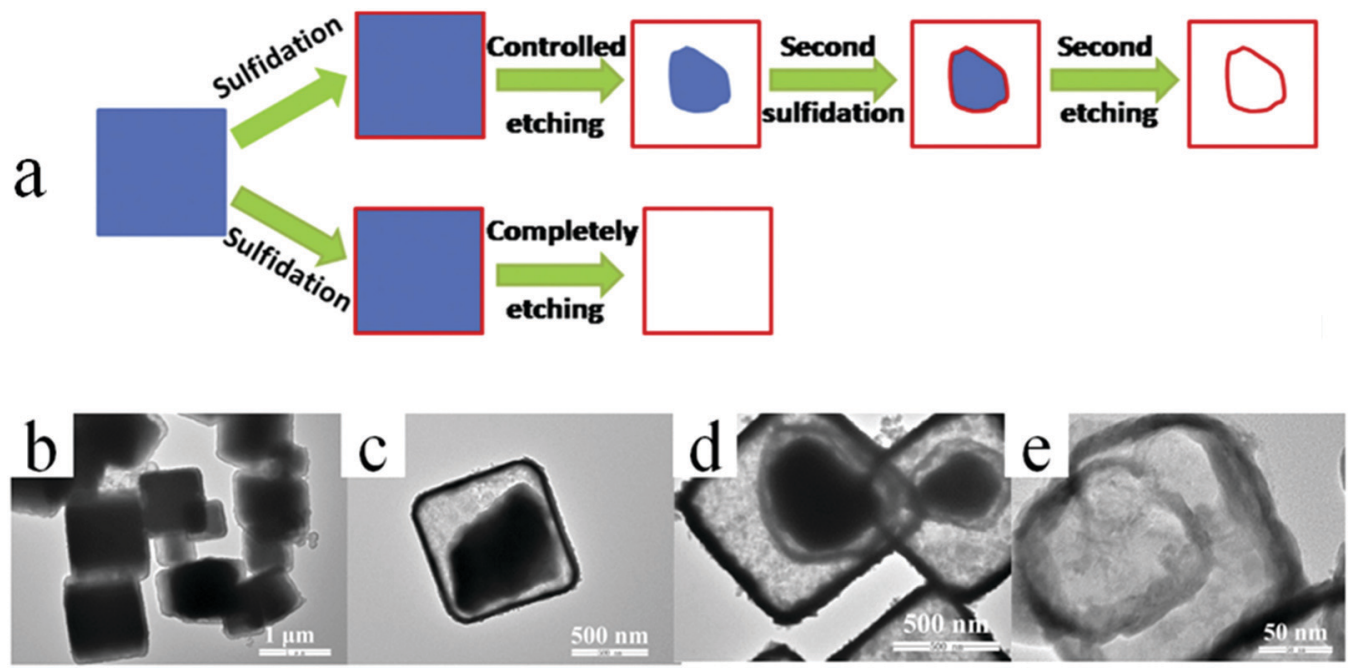

Fig. 30 (a) Schematic illustration of the synthesis procedure for the formation of double-shelled CuS hollow nanocages via the $\mathrm{Cu}_{2} \mathrm{O}$ self-template approach. TEM images of $\mathrm{Cu}_{2} \mathrm{O} @ \mathrm{CCuS}$ : (b) before and (c) after the initial partial etching treatment. TEM images of (d) $\mathrm{Cu} 2 \mathrm{O} @ \mathrm{CuS}$ CCuS and (e) doubleshelled CuS hollow nanocages. Reprinted from ref. 109 with kind permission. 


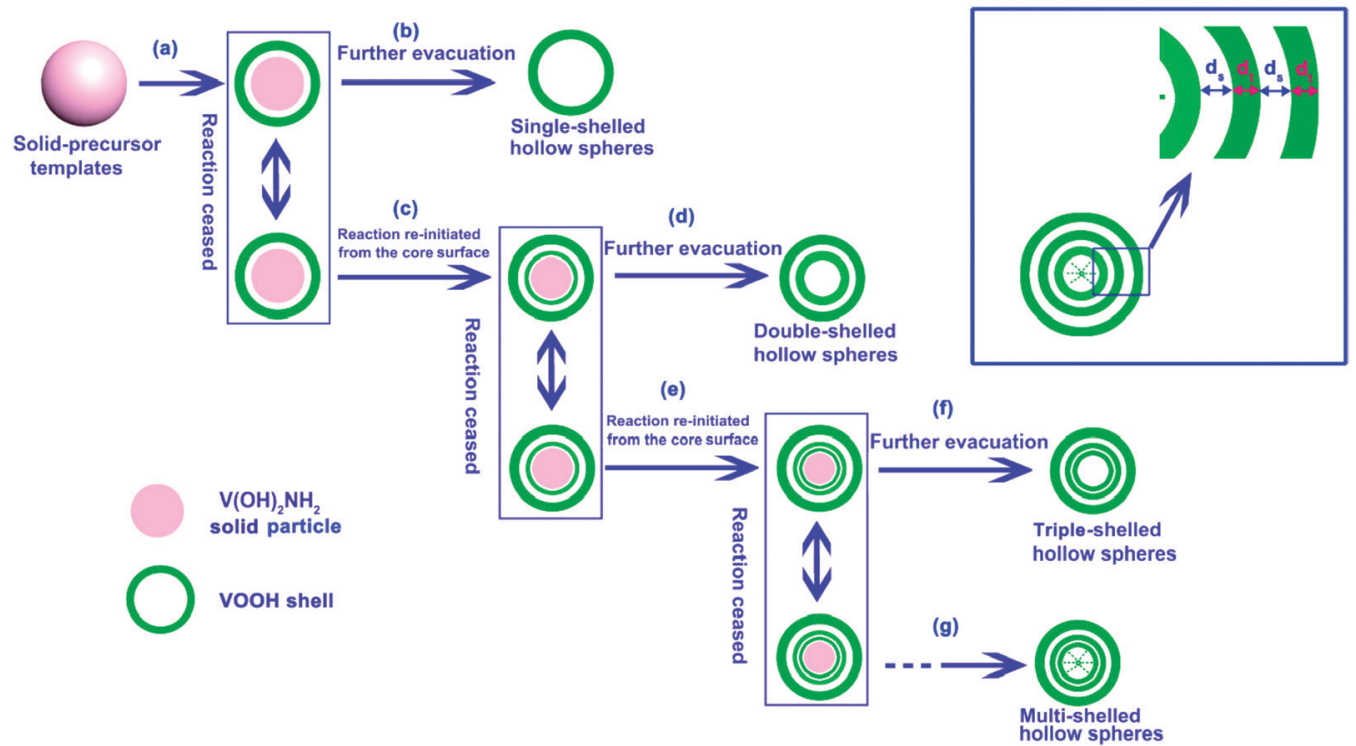

Fig. 31 Schematic illustration of the formation of $\mathrm{VOOH}$ hollow nanospheres with controlled numbers of shells via the direct thermal treatment of solid $\mathrm{V}(\mathrm{OH})_{2} \mathrm{NH}_{2}$ nanospheres. Reprinted from ref. 110 with kind permission.

hollow nanostructures with controlled sizes are preferable than other methods. ${ }^{112,113}$ Spray pyrolysis is a typical template-free method for preparing nanomaterials with unique structures. Zhou et al. described a spray-drying technique by adding glucose as a carbon source to prepare multishelled $\alpha-\mathrm{Fe}_{2} \mathrm{O}_{3}$ hollow spheres. ${ }^{114}$ The fabrication mechanism of multishelled $\alpha-\mathrm{Fe}_{2} \mathrm{O}_{3}$ hollow spheres may be explained as follows: the iron nitratesucrose system possessed several disadvantages when used as a precursor to prepare multishelled $\alpha-\mathrm{Fe}_{2} \mathrm{O}_{3}$ hollow spheres. Due to the nitrogen-rich contents, the thermal treatment of iron nitrate could generate nitrogen oxides (such as $\mathrm{NO}$ and $\mathrm{NO}_{2}$ ), which are harmful to the environment as well as human beings. Further, iron nitrate readily absorbs moisture from air, complicating the storage of the iron nitrate-sucrose composite. Furthermore, the strong oxidative property of iron nitrate and strong reductive property of sucrose in a single system may result in safety hazards, such as explosions. In addition, sucrose/iron nitrate ratio ranging only within $\sim 0.75-1$ may lead to multishelled $\alpha-\mathrm{Fe}_{2} \mathrm{O}_{3}$ hollow spheres, thereby restricting mass production. Considering the above concerns, Padashbarmchi et al. proposed another spraydrying method by replacing iron nitrate with iron citrate. ${ }^{115}$ It is well known that iron citrate is a mild oxidant and avoids the abovementioned limitations since the nitrogen content of the compound is zero. Using different metallic salts and additive carbon sources, other metallic oxides with multishelled hollow structures may be obtained. For example, Park et al. successfully used cobalt nitrate and ethylene glycol to prepare multishelled $\mathrm{Co}_{3} \mathrm{O}_{4}$ powders. ${ }^{116}$

From the literature, it is evident that the triaxial electrospinning technique is a versatile strategy to create multilayered structures. Inspired by this principle, Zanjani and co-workers fabricated multiwalled hollow fibers using a direct, one-step triaxial electrospinning process. In order to control the hydrophilicity of the inner and outer layers of the fiber, the polarities and viscosities of two spinnable polymer solutions used as the inner and outer layers were seriously considered. Consequently, the polymer concentrations play important roles in providing composite properties toward a wide range of applications. Furthermore, by adjusting the solvent properties and the degree of miscibility of solutions as well as the multiaxial electrospinning parameters (including applied voltage, flow rate, and electrospinning distance), multiwalled hollow fibers with the desired diameters, surface morphology, and layered structures could be obtained. ${ }^{117}$

The largest advantage of the template-free approach might be the fact that no tedious procedures are required, which is the necessary procedure for hard template approaches. When compared with soft template approaches, template-free ones might be preferred in controlling the particle size, number of shells/shell thickness, and morphology. With regard to the disadvantages, the exact formation mechanism of multishelled hollow structures by various template-free approaches is still unclear and needs to be further studied because of the difficulty in obtaining direct evidence by experiments.

\subsection{Multimethod combination approaches}

With the development of nanotechnology, some novel strategies may not be included in the four categories discussed above. Table 1 summarizes the advantages and disadvantages of the abovementioned methods. In the future, we can expect an integrated approach for synthesizing multishelled hollow nanostructures with desirable compositions and structural features, which cannot be directly fabricated by any single technique. When Zhang's work is considered as an example, both carbon nanofibers (CNFs) and metal glycolates were employed to form a series of multishelled metal oxide nanotubes. Apparently, CNFs and metal glycolates were used as the hard template and self-template, respectively. ${ }^{118}$ Meanwhile, owing to the inherent complications in a multishelled hollow 
Table 1 Summary of the advantages and disadvantages of several typical methods discussed in this review

\begin{tabular}{|c|c|c|}
\hline Methods & Advantages & Disadvantages \\
\hline $\begin{array}{l}\text { Hard tem- } \\
\text { plate method }\end{array}$ & $\begin{array}{l}\text { Efficient in controlling dimensions and function- } \\
\text { alities, available for numerous systems. }\end{array}$ & $\begin{array}{l}\text { Tedious operation procedures, shell number often limited less than } 5 \text {, } \\
\text { template removal needed, time- and/or energy-consuming, difficulty in } \\
\text { large scale production. }\end{array}$ \\
\hline $\begin{array}{l}\text { Soft template } \\
\text { method }\end{array}$ & $\begin{array}{l}\text { Leading to uniform shape and sizes of the product. } \\
\text { Usually performed under mild condition. }\end{array}$ & $\begin{array}{l}\text { Organic molecule assembly affects the purity of the product. Consuming } \\
\text { plenty of organic solvent/compound. }\end{array}$ \\
\hline $\begin{array}{l}\text { Self-template } \\
\text { method }\end{array}$ & $\begin{array}{l}\text { Shell number reach up to or over } 5 \text {, simple } \\
\text { procedure, time and cost efficient. }\end{array}$ & $\begin{array}{l}\text { Much dependent on the properties of precursors, need precise control over } \\
\text { experimental condition, incapable of wide utilization, the shell number of } \\
\text { the product cannot be accurately controlled. }\end{array}$ \\
\hline $\begin{array}{l}\text { Template-free } \\
\text { method }\end{array}$ & $\begin{array}{l}\text { No complicated operation needed, usually simple } \\
\text { heat treatment or spray drying does work, shell } \\
\text { number/thickness and morphology of the final } \\
\text { products can be well controlled. }\end{array}$ & $\begin{array}{l}\text { The exact mechanism is subject to further studies. Fewer precursor systems } \\
\text { available for this method. }\end{array}$ \\
\hline
\end{tabular}

structure, a combination of multiple methods may involve several mechanisms in a single system, such as ionic exchange, Ostwald ripening, Kirkendall effect, self-assembly, successive solid deposition, ${ }^{119}$ seed-mediated successive Ostwald ripening, ${ }^{120,121}$ and so on. Readers interested in these cases are encouraged to refer to the specific examples. ${ }^{122}$

\section{Applications of multishelled hollow nanostructures}

Multishelled hollow nanostructures, integrating the advantages of both hollow materials and multiple-shelled architectures, are expected to realize their optimized physical/chemical properties for specific applications. The unique characteristics of multishelled hollow structures render them to have widespread applications in energy conversion and storage, environmental treatment, catalysis, and biomedicine. The applications of multishelled hollow nanostructure in various areas are summarized in this section.

\subsection{Energy-related applications}

With the depletion of fossil fuels and exploration of renewable sustainable clean energy resources, the electrical energy storage devices, such as supercapacitors, different ionic batteries, dyesensitized solar cells, etc., have received extensive attention. As a result, there is an urgent need to develop high-performance electrodes for use in such devices. Superior over traditional singleshelled hollow nanostructures with regard to specific surface area and surface-to-mass/volume ratios, multishelled hollow nanostructures have the ability to increase the weight fraction of the active species, boosting the volumetric energy/power density, and prolonging cycling life.

3.1.1 Supercapacitors. With the rapid consumption of traditional nonrenewable fossil energy (coal, petroleum, and natural gas) and ever-worsening environmental pollution, some novel clean renewable energy resources, such as wind energy, solar energy, and so on, are expected to substitute traditional fossil energies. The design and construction of high-performance energy storage devices is the subject of immense importance, particularly for exploring novel energy resources. ${ }^{123-126}$ When compared with other energy storage devices, low cost, fast charge-discharge capability, high power density, and long cycling life have made supercapacitors to be good candidates for energy storage devices. ${ }^{127-130}$ Until now, carbon-based materials (such as carbon nanotubes, graphene, carbon nanofibers, hierarchically porous carbons), transition metal oxides/ sulfides/nitrides, and conducting polymers, as well as various composites of these materials, have been demonstrated to be promising electrode materials for use in supercapacitors. ${ }^{131-139}$

Considering their superior environmental compatibility, specific capacitance, and cost-effectiveness, some transition metal oxides, such as copper oxide, iron oxide, nickel oxide, cobalt oxide, and manganese oxide, have been widely investigated as supercapacitor electrode materials. ${ }^{140-147}$ In order to improve the amount of electrochemically active sites, enhance electron and ion conductivities, as well as inhibit the dissolution of active species into the electrolytes, various hollow metal oxide nanostructures have been preferred. For example, Wang et al. compared the electrochemical performances of $\mathrm{Mn}_{2} \mathrm{O}_{3}$ nanoparticles, as well as single-, double-, triple-, and quadruple-shelled $\mathrm{Mn}_{2} \mathrm{O}_{3}$ hollow nanospheres when used as supercapacitor electrode materials. Their research results revealed that triple-shelled $\mathrm{Mn}_{2} \mathrm{O}_{3}$ hollow nanospheres exhibited better supercapacitive performance than $\mathrm{Mn}_{2} \mathrm{O}_{3}$ nanoparticles and single-, double-, and quadruple-shelled counterparts. ${ }^{43}$ Yang et al. prepared single-, double-, and triple-shelled NiO hollow nanospheres through layer-by-layer self-assembly and subsequent calcination; their electrochemical performances were studied when used as supercapacitor electrodes. ${ }^{148}$ Their results showed that the NiO hollow nanosphere sample with double shells exhibited the best electrochemical properties among the three NiO hollow nanosphere samples, such as the highest specific capacitance of $612.5 \mathrm{~F} \mathrm{~g}^{-1}$ at $0.5 \mathrm{~A} \mathrm{~g}^{-1}$ and long-term cycling stability with retention of over $90 \%$ of the initial specific capacitance after 1000 charge-discharge cycles. They attributed such distinguishable performance to two factors: larger active surface area of $\mathrm{NiO}$ with the double-shelled structure assured the bulk accessibility of faradaic reactions and the loosely assembled thin nanoflakes of the double-shelled hollow NiO exhibited a porous structure that shortened the ion diffusion path for effective electrolyte diffusion.

Besides single-metal oxides, numerous studies have demonstrated that mixed metallic oxides are expected to deliver 
superior electrochemical performance as compared to singlemetal oxides. ${ }^{149-151}$ Qi et al. reported thin/thick single-/double-/ triple-shelled $\mathrm{NiCo}_{2} \mathrm{O}_{4}$ hollow microspheres used as electrodes for electrochemical supercapacitors. ${ }^{61}$ Moosavifard et al. demonstrated that the coordination polymer of isophthalic acid and various metallic ions are versatile precursors to obtain multishelled hollow nanostructures, including $\mathrm{CuCo}_{2} \mathrm{O}_{4}$ and mixed copper cobalt phosphide. These multishelled mixed metal oxides and phosphides were demonstrated to be promising electrode material candidates for asymmetric supercapacitor (ASC) devices. ${ }^{152,153}$

In addition to metal oxides, metal sulfides have also been widely applied as supercapacitor electrodes. When compared to oxides, transition metal sulfides are expected to exhibit greater electric conductivity and better redox chemistry. ${ }^{154}$ In order to improve the rate capability and cycling stability, the design and fabrication of the hierarchical nanostructure is demonstrated to be an effective way. Du et al. prepared three types of doubleshelled Nis hollow nanostructures with different morphologies (cube-, ellipsoid-, and capsule-shaped), and they investigated their electrochemical performances when used as supercapacitor electrodes. Their results revealed that the double-shelled NiS hollow nanostructures in the shape of a capsule, having the highest specific surface area of $100.2 \mathrm{~m}^{2} \mathrm{~g}^{-1}$ and the lowest equivalent series resistance of $0.8 \Omega$, offered the largest specific capacitance of $1159 \mathrm{~F} \mathrm{~g}^{-1}$ at a current density of $2 \mathrm{~A} \mathrm{~g}^{-1}$ among all the three as-prepared samples. ${ }^{25}$

Another study by Lou et al. reported the enhanced pseudocapacitive properties of nickel cobalt sulfide ball-in-ball hollow spheres. ${ }^{106}$ When used as an electrode in electrochemical capacitors, the as-obtained $\mathrm{NiCoS}_{4}$ ball-in-ball hollow spheres delivered high specific capacitance of $1036 \mathrm{~F} \mathrm{~g}^{-1}$ at $1 \mathrm{~A} \mathrm{~g}^{-1}$. Furthermore, they constructed an ASC device using the asobtained $\mathrm{NiCoS}_{4}$ hollow sample as the cathode and graphene/ carbon sphere paper as the anode (Fig. 32a). ${ }^{19}$ The performance of this ASC device was systematically investigated by using an array of techniques, such as cyclic voltammetry (CV) and galvanostatic charge-discharge (GCD). The authors found that the entire capacitance of the $\mathrm{NiCoS}_{4}$ ball-in-ball hollow sample was contributed by both electric double-layer capacitance and pseudocapacitance. Meanwhile, higher scan rates resulted in higher current densities, which is a common behavior of a typical electrochemical system. The shape of the CV curves is independent of the scan rate, showing no obvious distortion; this implied good and fast charge-discharge properties of the device. The specific capacitance values at various scan rates indicated the excellent rate capacity of the constructed ASC device. The cycling performance of the ASC device is shown in Fig. 32b. It is apparent that the ASC device offers very high cycling stability with retention of $78.6 \%$ of its initial capacitance at the end of 10000 cycles. The authors also evaluated the energy and power densities of the ASC device and demonstrated that the ASC device exhibited superior balance between the energy density and power density as compared to many of the reported cobalt-/nickel-based ASCs. ${ }^{155-158}$ By changing the species of metal cations, Wei et al. prepared double-shelled $\mathrm{ZnS}-\mathrm{NiS}_{1.97}$ hollow spheres by using a similar procedure, which possessed a porous structure and high specific surface area $\left(105.26 \mathrm{~m}^{2} \mathrm{~g}^{-1}\right)$. When used as an electrode material for a supercapacitor, the double-shelled $\mathrm{ZnS}^{-N_{i} \mathrm{~S}_{1.97}}$ hollow spheres delivered excellent electrochemical performance in terms of specific capacitance (696.8 $\mathrm{C} \mathrm{g}^{-1}$ at $5 \mathrm{~A} \mathrm{~g}^{-1}$ ), cycling stability (less than $5.5 \%$ loss after 6000 cycles), and energy density of $25.6 \mathrm{~W} \mathrm{~h} \mathrm{~kg}^{-1}$ at a power density of $2173.8 \mathrm{~W} \mathrm{~kg}^{-1} \cdot{ }^{159}$ Similar to oxides, ternary metal sulfides with complex double-shelled structures were also expected to provide a superior electrochemical performance over single-metal oxides. Considering the work of Lou's group as an example, they reported that $\mathrm{NiCo}_{2} \mathrm{O}_{4}$ double-shelled hollow spheres, as a typical battery-like electrode, offered specific capacitance as high as $1036 \mathrm{~F} \mathrm{~g}^{-1}$ at $1 \mathrm{~A} \mathrm{~g}^{-1}$. Significantly, the ASC device (Fig. 32), assembled by using $\mathrm{NiCo}_{2} \mathrm{~S}_{4}$ hollow spheres and graphene/mesoporous carbon sphere composite, delivered a high energy density of $42.3 \mathrm{~W} \mathrm{~h} \mathrm{~kg}^{-1}$ at a power density of $476 \mathrm{~W} \mathrm{~kg}^{-1}$. Furthermore, the assembled ASC device also showed excellent cycling stability, retaining $78.6 \%$ of the initial capacitance after 10000 cycles at $5 \mathrm{~A} \mathrm{~g}^{-1}$.

It is easy to consider the construction of the heterostructures of oxides/sulfides for use as supercapacitor electrodes since both oxides and sulfides are promising electrode materials owing to the high theoretical specific capacitance and high electrochemical activity. By the calcination of hollow $\mathrm{Co}_{3} \mathrm{O}_{4}$
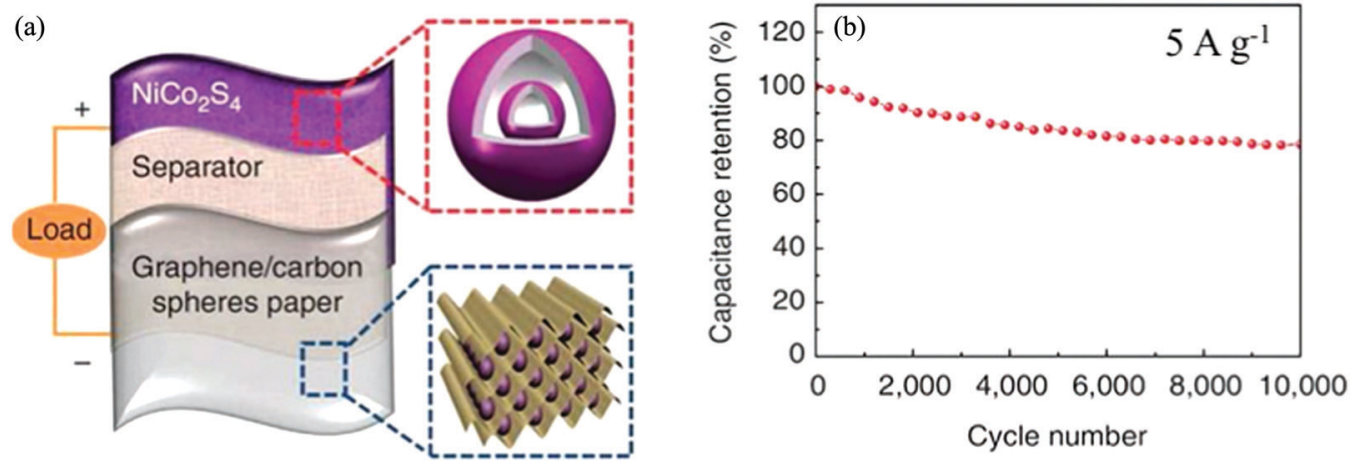

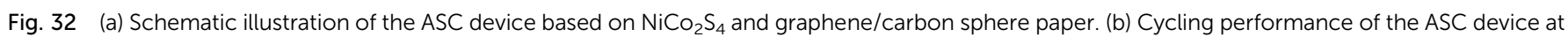
$5 \mathrm{~A} \mathrm{~g}^{-1}$. Reproduced from ref. 19 with permission. 
hollow spheres with S powder in Ar gas, Wang et al. successfully prepared multishelled $\mathrm{CoO} @ \mathrm{Co}_{9} \mathrm{~S}_{8}$ hollow microspheres. ${ }^{160}$ These multishelled $\mathrm{CoO} @ \mathrm{Co}_{9} \mathrm{~S}_{8}$ hollow microspheres delivered high specific capacitance of $1100 \mathrm{~F} \mathrm{~g}^{-1}$ at $2 \mathrm{~A} \mathrm{~g}^{-1}$, good rate capability (809 $\mathrm{F} \mathrm{g}^{-1}$ at $50 \mathrm{~A} \mathrm{~g}^{-1}$ ), and excellent cycling stability (from 1035 to $785 \mathrm{~F} \mathrm{~g}^{-1}$ after 2000 cycles at $2 \mathrm{~A} \mathrm{~g}^{-1}$ ) when used as an electrode material. Such performances are superior to those of previously reported cobalt-based oxide and sulfide electrodes. When coupled with activated carbon to construct an ASC device, the device shows faradaic characteristics in the CV curves and delivers good rate capability and high capability retention of $95.27 \%$ after 5000 cycles. Recently, they extended this method to prepare multishelled NiS@NiO hollow heterostructures, which also exhibited excellent electrochemical performance when used as the electrode in ASC devices. ${ }^{161}$ The excellent performance of these multishelled oxide@sulfide hollow heterostructures could be effectively indexed into the following aspects. First, the introduction of sulfur species could enhance the electrical conductivity, leading to improved charge transport. Second, oxide/sulfide heterostructures provide different bandgaps that facilitate the surface reaction kinetics and charge transfer between the electrode/electrolyte interfaces. Last, but not the least, the interlayer spaces in the multishelled hollow structures can act as reservoirs to accommodate more electrolytes and increase the contact area between the electrode and electrolyte.

As mentioned earlier, carbon is also a promising electrode material for use in supercapacitors. Using crosslinked PS and silica as a dual hard template and DA as a N-containing carbon precursor, Fang et al. fabricated uniform discrete doubleshelled N-doped carbon hollow cage-like spheres. When used as a supercapacitor electrode, the specific capacitance values of the double-shelled N-doped carbon hollow cage-like spheres reached as high as 229.3 and $160 \mathrm{~F} \mathrm{~g}^{-1}$ at 1 and $10 \mathrm{~A} \mathrm{~g}^{-1}$, respectively. Meanwhile, owing to the superior 3D hierarchically porous structure, both rate capability and cycling stability were excellent. ${ }^{162}$

It is well known that three parameters, namely, energy density, power density, and cycling life, are the most important. On the basis of the above examples, it can be concluded that the structural characteristics of multishelled hollow nanostructures are expected to have a considerable influence on the performance of supercapacitors. Generally, multiple thin shells usually provide high volumetric energy density owing to the large surface-to-volume ratio, good electrode/electrolyte contact, and abundant active sites. The porous shells facilitate electronic/ionic transformation, leading to high power density. The intershell space with optimized void-to-solid ratio is a significant factor that determines the cycling stability of multishelled hollow nanostructures when used as supercapacitor electrodes.

3.1.2 Alkali metal-ion batteries. Except the engine system, the power source is one of the most important components, which, to a great extent, comprises the major cost of vehicles. In view of environmental protection, LIBs are expected to manipulate the power source of electric vehicles and hybrid electric vehicles. ${ }^{163-165}$ The most vital task is to develop highperformance LIBs as promising anode materials with high capability, no memory effect, low self-discharge rate, and longtime cycling stability. Transition metal compounds and some main group metal compounds possess higher theoretical capacity (over $600 \mathrm{~mA} \mathrm{~h} \mathrm{~g}^{-1}$ ) than commercial graphite $\left(372 \mathrm{~mA} \mathrm{~h} \mathrm{~g}{ }^{-1}\right.$ ). Nevertheless, rapid capacity decay and poor capacity retention caused by their severe volume expansion and loss of electrical conductivity are the result of pulverization and deterioration of the electrode materials during $\mathrm{Li}^{+}$insertion and extraction.

Recently, a considerable amount of effort has been devoted toward exploring alternative electrode materials that can deliver high capacity and good rate capability. In this context, metal oxides have emerged as promising candidates for use as anode materials for LIBs in view of their relatively low cost, nontoxicity, higher theoretical capacity, and intrinsically enhanced safety. ${ }^{166,167}$ Multishelled hollow nanostructures, possessing advantages similar to other nanomaterials and unique advantages of their hollow cavities, may offer not only plentiful electrodeelectrolyte reaction sites but also a "buffer" to restrict the volume expansion during the $\mathrm{Li}^{+}$charge-discharge process. Using histidine as a structure-directing agent, Wu et al. developed a facile route based on a hydrothermal process and subsequent thermal treatment in air to prepare porous multishelled $\alpha-\mathrm{Fe}_{2} \mathrm{O}_{3}$ hollow spheres. The unique porous multishelled $\alpha-\mathrm{Fe}_{2} \mathrm{O}_{3}$ hollow spheres possessed a large specific surface area of $14.2 \mathrm{~m}^{2} \mathrm{~g}^{-1}$ and they offered a high specific capacity, excellent cycling stability, and high rate capability when applied as anode for LIBs. The electrochemical performance was demonstrated to be superior as compared to those of various $\mathrm{Fe}_{2} \mathrm{O}_{3}$-based anode materials reported earlier. This is due to their unique structural advantages, such as easy to be filtrated in the electrolyte, more locations to store lithium ions, and short ion diffusion path. ${ }^{168} \mathrm{Xu}$ et al. prepared multishelled $\alpha-\mathrm{Fe}_{2} \mathrm{O}_{3}$ hollow nanospheres with controlled number of shells and thickness by adjusting the solvent composition through a carbonaceous template method. ${ }^{36}$ As shown in Fig. 33, the number and thickness of shells could be controlled by adjusting the experimental parameters. When used as an anode for LIBs, the authors found that both number of shells and shell thickness have a significant impact on the energy storage performance. Apparently, thin triple-shelled samples deliver the best performance.

Cobalt oxide has also been demonstrated as a promising electrode candidate for LIBs. As shown in Fig. 34a, the initial charge-discharge profiles show a distinct voltage plateau and long tailing. Different numbers of shells of the $\mathrm{Co}_{3} \mathrm{O}_{4}$ hollow spheres lead to different charge-discharge times. Apparently, triple-shelled structures deliver the highest energy density. With regard to the cycling performance (Fig. 34b), multishelled $\mathrm{Co}_{3} \mathrm{O}_{4}$ hollow spheres exhibit superior specific capacity than that of a commercial sample. The reversible capacities of single-, double-, triple-, and quadruple-shelled $\mathrm{Co}_{3} \mathrm{O}_{4}$ microspheres are much higher than that of commercial $\mathrm{Co}_{3} \mathrm{O}_{4}$.

In another work, Yin and co-workers prepared $\mathrm{Co}_{3} \mathrm{O}_{4}$ hollow spheres with tunable numbers of shells (from yolk-shell to multishell) by forming cobalt complex microspheres via a 

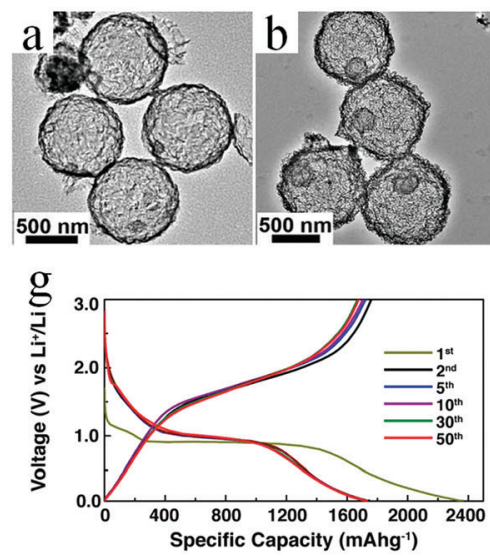
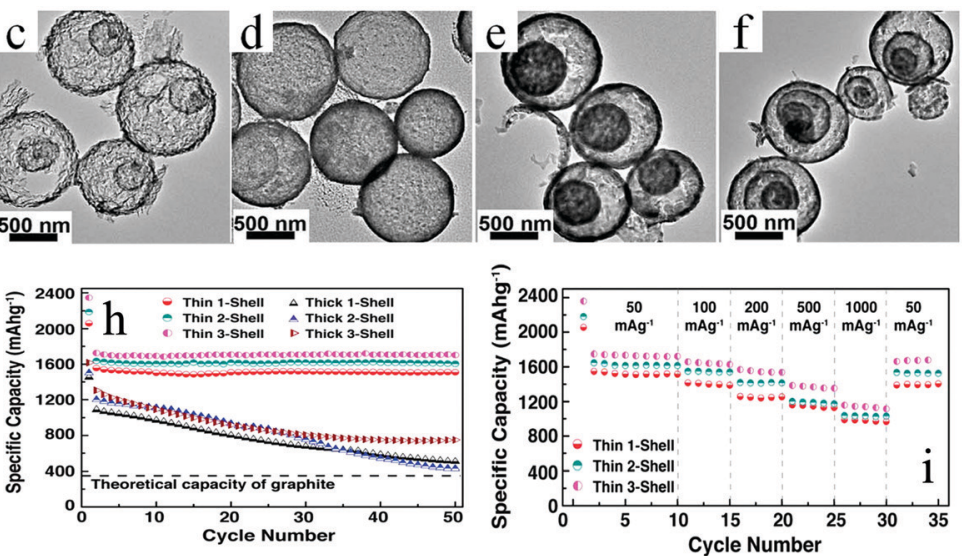

Fig. 33 (a-f) TEM images of multishelled $\alpha-\mathrm{Fe}_{2} \mathrm{O}_{3}$ hollow nanospheres with different numbers of shells and shell thicknesses: (a) thin single-shelled, (b) thin double-shelled, (c) thin triple-shelled, (d) thick single-shelled, (e) thick double-shelled, and (f) thick triple-shelled samples. ( $\mathrm{g}$-i) Electrochemical performance of above samples. Reprinted from ref. 36 with kind permission.
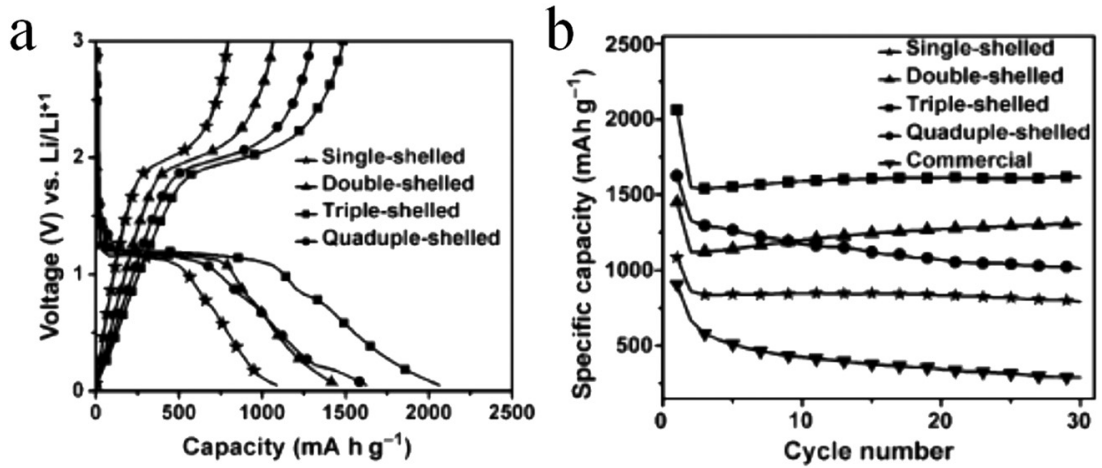

Fig. 34 (a) Initial discharge-charge curves for $\mathrm{CO}_{3} \mathrm{O}_{4}$ hollow microspheres with different numbers of shells. (b) Discharge capacity versus number of cycles for $\mathrm{CO}_{3} \mathrm{O}_{4}$ hollow microspheres with different numbers of shells and commercial $\mathrm{CO}_{3} \mathrm{O}_{4}$. Reprinted from ref. 40 with permission. Copyright 2013 John Wiley \& Sons.

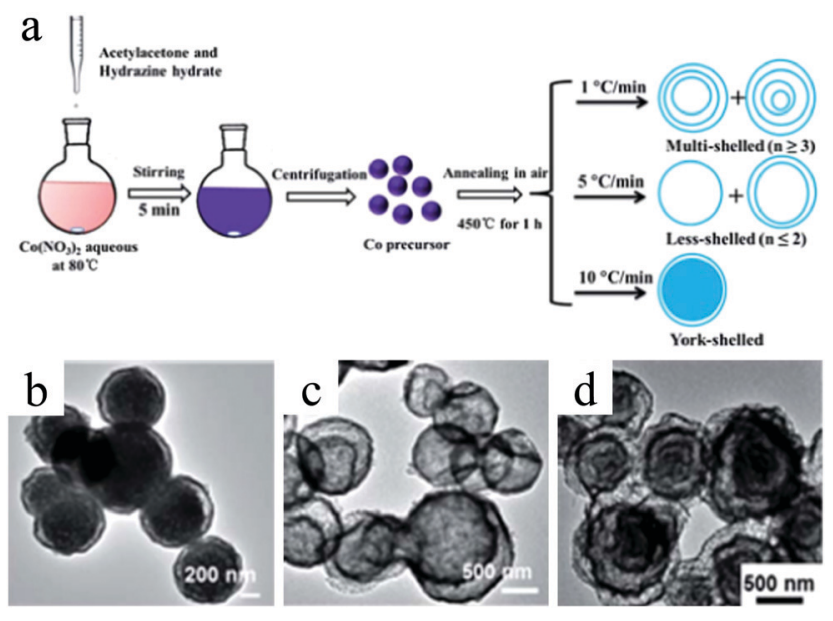

Fig. 35 (a) Schematic illustration of the synthesis process and (b-d) typical TEM images of $\mathrm{CO}_{3} \mathrm{O}_{4}$ hollow spheres in yolk-shell, fewer-shelled, and multishelled structures. Reproduced from ref. 169 with permission.

solution-based route under mild conditions and subsequent controlled calcination processes in air (Fig. 35). ${ }^{169}$ Fig. 36 shows the electrochemical performance of the three as-obtained $\mathrm{Co}_{3} \mathrm{O}_{4}$ samples. It has been demonstrated that multishelled $\mathrm{Co}_{3} \mathrm{O}_{4}$ hollow spheres possessed excellent storage properties with a high specific capacity $\left(1379.6 \mathrm{~mA} \mathrm{~h}{ }^{-1}\right)$, good rate capability $\left(1026 \mathrm{~mA} \mathrm{~h} \mathrm{~g}^{-1}\right.$ at $0.1 \mathrm{~A} \mathrm{~g}^{-1}$ and $769 \mathrm{~mA} \mathrm{~h} \mathrm{~g}^{-1}$ at $\left.5 \mathrm{~A} \mathrm{~g}^{-1}\right)$, and excellent cycling stability $\left(1058 \mathrm{~mA} \mathrm{~h} \mathrm{~g}^{-1}\right.$ at $1 \mathrm{~A} \mathrm{~g}^{-1}$ after 1000 cycles). Such values are better than fewer-shelled and yolk-shell structures.

In order to further improve the lithium storage properties of $\mathrm{Co}_{3} \mathrm{O}_{4}$, Yu et al. demonstrated an effective strategy to synthesize hollow $\mathrm{Co}_{3} \mathrm{O}_{4} @ \mathrm{Co}_{3} \mathrm{~V}_{2} \mathrm{O}_{8}$ hybrid nanoboxes using the reactivity of ZIF-67 with the vanadium source of vanadium oxytriisopropoxide. When tested as an anode for LIBs, triple-shelled hollow $\mathrm{Co}_{3} \mathrm{O}_{4} @ \mathrm{Co}_{3} \mathrm{~V}_{2} \mathrm{O}_{8}$ hybrid nanoboxes demonstrated a remarkable initial discharge capacity of $1909 \mathrm{~mA} \mathrm{~h} \mathrm{~g}^{-1}$, reversible charge capacity of $1186 \mathrm{~mA} \mathrm{~g}^{-1}$, and good stability with capacity retention of $948 \mathrm{~mA} \mathrm{~h} \mathrm{~g}^{-1}$ after 100 cycles at $100 \mathrm{~mA} \mathrm{~g}^{-1} \cdot{ }^{170}$

Similar to iron oxides and cobalt oxides, nickel oxides have also received considerable attention as anode materials for LIBs because of their high theoretical capacity $\left(718 \mathrm{~mA} \mathrm{~h} \mathrm{~g}{ }^{-1}\right)$, natural abundance, and nontoxicity. ${ }^{171,172}$ Li et al. improved the lithium storage properties by constructing multishelled NiO hollow microspheres for use as the anode. They found 

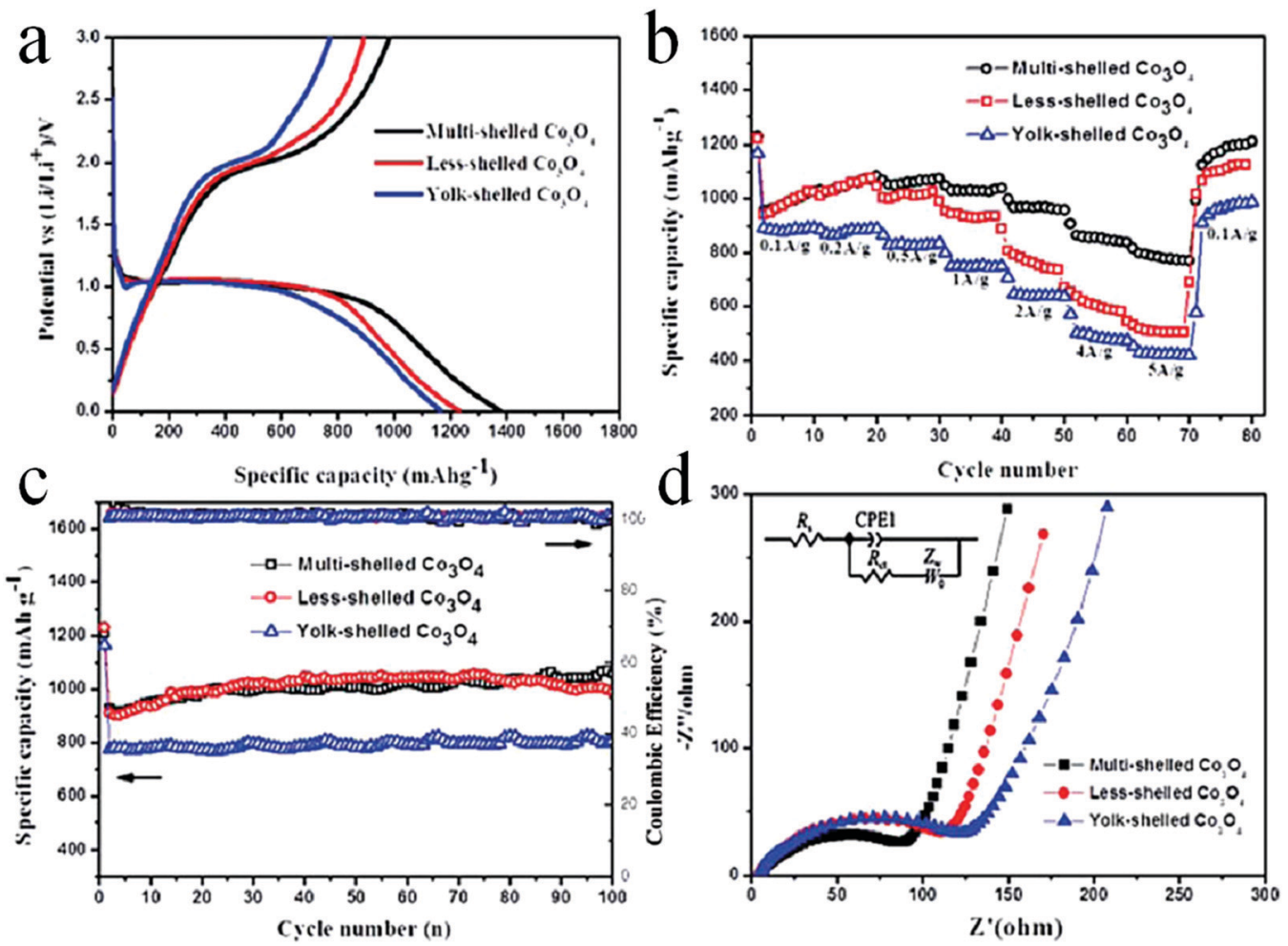

Fig. 36 Electrochemical performance of $\mathrm{CO}_{3} \mathrm{O}_{4}$ hollow spheres in yolk-shell, fewer-shelled, and multishelled structures when used as the anode for LIBs. (a) Initial charge-discharge curves, (b) rate capability for different current densities, (c) cycling performance at $1 \mathrm{~A} \mathrm{~g}^{-1}$, and (d) electrochemical impedance spectra. Reproduced from ref. 169 with permission.

that triple-shelled NiO hollow microspheres exhibited high specific capacity, outstandingly high rate capability, as well as excellent cycling performance. After 100 discharge-charge cycles, the triple-shelled NiO hollow spheres could deliver a reversible capacity of $\sim 789 \mathrm{~mA} \mathrm{~h} \mathrm{~g}{ }^{-1}$ at a current density of $500 \mathrm{~mA} \mathrm{g^{-1 }}$. Significantly, the specific discharge capacity of porous triple-shelled NiO hollow spheres retained up to $721 \mathrm{~mA} \mathrm{~h} \mathrm{~g}^{-1}$ even at a high current density of $2000 \mathrm{~mA} \mathrm{~g}^{-1}$. The excellent electrochemical performance could be indexed to the self-supporting multishelled hollow microstructure, guaranteeing ample sites for storing lithium, shortening the lithium-ion diffusion length, and providing adequate void space for buffering the volume expansion. ${ }^{173}$

Vanadium-based oxides possessing diverse oxidation states, such as $\mathrm{V}_{2} \mathrm{O}_{5}, \mathrm{VO}_{2}, \mathrm{~V}_{4} \mathrm{O}_{7}$, and $\mathrm{V}_{2} \mathrm{O}_{3}$, have also been demonstrated as versatile lithium-ion intercalation hosts. ${ }^{174}$ Among vanadium-based oxides, $\mathrm{V}_{2} \mathrm{O}_{5}$ has been the most extensively investigated anode candidate for LIBs in the past decade.
Recently, Wang et al. prepared multiple double-walled $\mathrm{V}_{2} \mathrm{O}_{5}$ hollow nanospheres from a vanadium oxide precursor via an ascorbic-acid-assisted solvothermal process followed by an annealing treatment (Fig. 37). ${ }^{175}$ When evaluated as an electrode for LIBs, the obtained multishelled $\mathrm{V}_{2} \mathrm{O}_{5}$ hollow nanospheres exhibited excellent performance (Fig. 38). The data shown in Fig. 38a have two cathodic peaks at 3.36 and $3.17 \mathrm{~V}$, indicating the intercalation of lithium ions into the electrode materials at two steps and yielding two new phases, namely, $\mathrm{Li}_{0.5} \mathrm{~V}_{2} \mathrm{O}_{5}$ and $\mathrm{LiV}_{2} \mathrm{O}_{5}$. The anodic peaks at 3.26 and $3.34 \mathrm{~V}$ can be attributed to the lithium deintercalation process and the phase changing back from $\mathrm{Li}_{0.5} \mathrm{~V}_{2} \mathrm{O}_{5}$ and $\mathrm{LiV}_{2} \mathrm{O}_{5}$ to $\mathrm{V}_{2} \mathrm{O}_{5}$. The $\mathrm{CV}$ results reveal that the lithium insertion process in the as-obtained multishelled $\mathrm{V}_{2} \mathrm{O}_{5}$ hollow nanospheres is completely reversible. The rate performance of the as-obtained multishelled $\mathrm{V}_{2} \mathrm{O}_{5}$ hollow nanospheres is shown in Fig. 38b. Evidently, the initial discharge capacity could reach up to $146 \mathrm{~mA} \mathrm{~h} \mathrm{~g}^{-1}$ at $50 \mathrm{~mA} \mathrm{~g}^{-1}$. This value is very close to the theoretical capacity of $\mathrm{V}_{2} \mathrm{O}_{5}$. When the current density

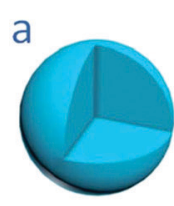

Precursor

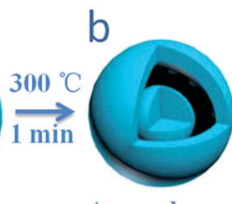

Amorphous

Vanadium/C

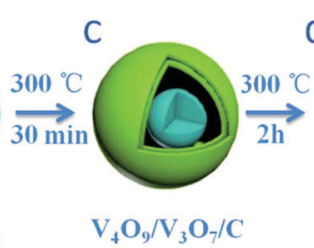

$\mathrm{V}_{4} \mathrm{O}_{9} / \mathrm{V}_{3} \mathrm{O}_{7} / \mathrm{C}$

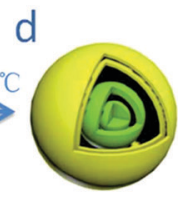

$\mathrm{V}_{2} \mathrm{O}_{5} / \mathrm{V}_{3} \mathrm{O}_{7} / \mathrm{C}$

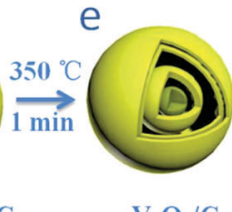

$\mathrm{V}_{2} \mathrm{O}_{5} / \mathrm{C}$

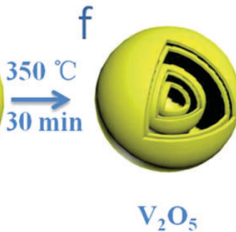

Fig. 37 Schematic illustration of the formation process of hollow $\mathrm{V}_{2} \mathrm{O}_{5}$ spheres with different interiors. Reproduced from ref. 175 with permission. 

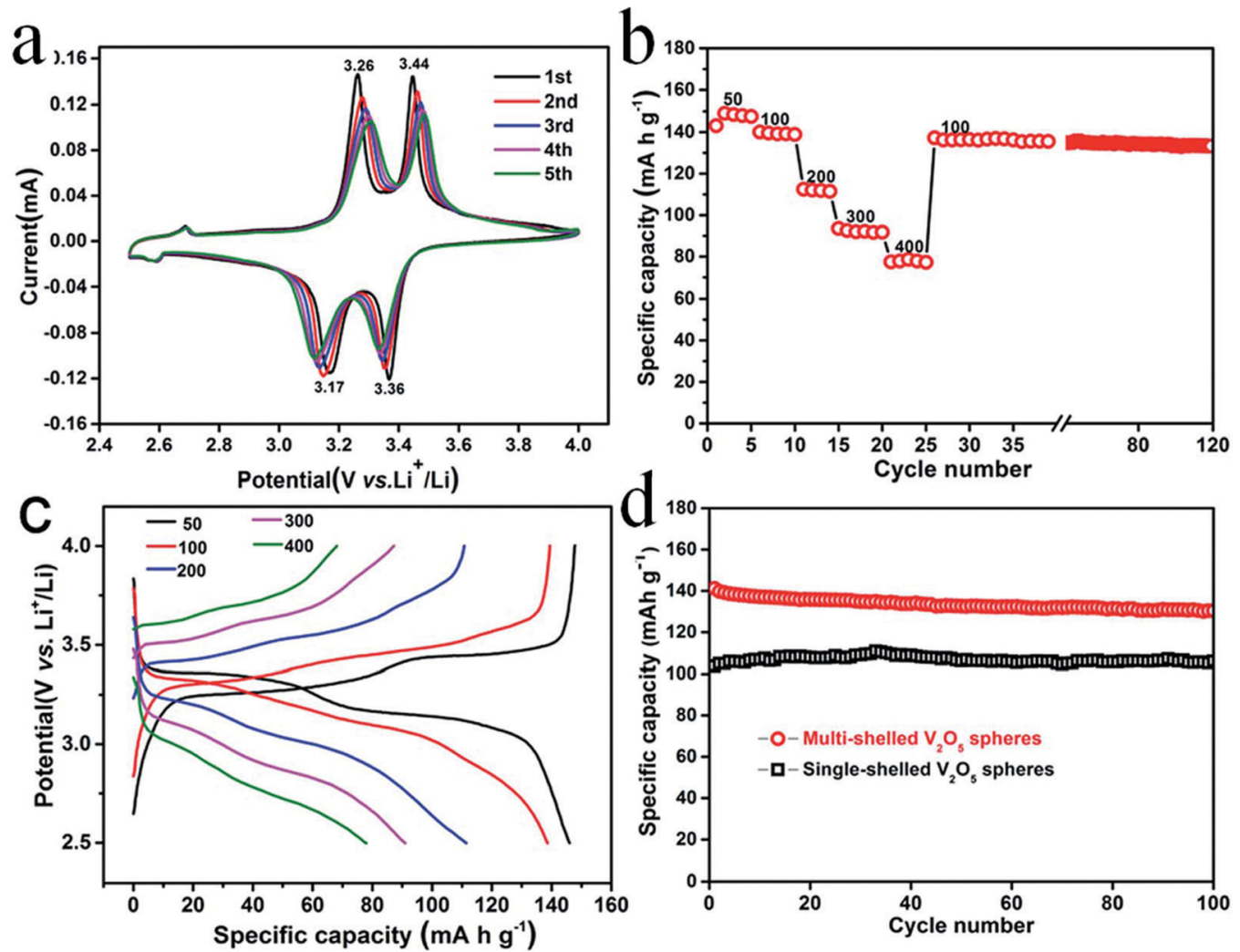

Fig. 38 Electrochemical performance of multishelled $\mathrm{V}_{2} \mathrm{O}_{5}$ hollow microspheres as the anode for LIBs. (a) CV profiles at $0.05 \mathrm{mV} \mathrm{s}^{-1}$, (b) rate performance, (c) charge-discharge curves at different current densities, and (d) cycling stability when compared with traditional single-shelled hollow samples at a current density of $100 \mathrm{~mA} \mathrm{~g}^{-1}$. Reproduced from ref. 175 with permission.

increased to $400 \mathrm{~mA} \mathrm{~g}^{-1}$, the capacity retained was $79 \mathrm{~mA} \mathrm{~h} \mathrm{~g}^{-1}$, indicating good rate capability of these multishelled $\mathrm{V}_{2} \mathrm{O}_{5}$ hollow nanospheres. Significantly, after 120 cycles, the capacity decay could be negligible (from 139 to $133 \mathrm{~mA} \mathrm{~h} \mathrm{~g}^{-1}$ ) at $100 \mathrm{~mA} \mathrm{~g}^{-1}$. Two plateaus observed in the charge-discharge curves (Fig. 38c) further demonstrated the good rate performance of multishelled $\mathrm{V}_{2} \mathrm{O}_{5}$ hollow nanospheres. When compared with single-shelled $\mathrm{V}_{2} \mathrm{O}_{5}$ hollow nanospheres, multishelled $\mathrm{V}_{2} \mathrm{O}_{5}$ hollow nanospheres exhibit superior cycling stability (Fig. 38d), which can be ascribed to the enlarged contact area between the electrode and electrolyte, resulting in the efficient penetration of the electrolyte.

Guo et al. exhaustively compared the electrochemical performances of $\mathrm{V}_{2} \mathrm{O}_{5}$ double-shelled and single-shelled hollow nanospheres as well as $\mathrm{V}_{2} \mathrm{O}_{5}$ nanoparticles prepared by thermally treating vanadyl glycerolate solid spheres. Their results reveal that the as-prepared double-shelled $\mathrm{V}_{2} \mathrm{O}_{5}$ hollow nanospheres can deliver an initial capacity of $256.7 \mathrm{~mA} \mathrm{~h} \mathrm{~g}{ }^{-1}$ with Coulombic efficiency of nearly $100 \%$, while the single-shelled and nanoparticle samples offer only 226.6 and $217 \mathrm{~mA} \mathrm{~h} \mathrm{~g}^{-1}$, respectively. Furthermore, the reversible residual capacity retention is $197.6 \mathrm{~mA} \mathrm{~h} \mathrm{~g}^{-1}$ after 50 cycles, which is superior as compared to those of single-shelled hollow nanospheres $\left(160.3 \mathrm{~mA} \mathrm{~h} \mathrm{~g}^{-1}\right)$ and solid nanoparticles $\left(145.5 \mathrm{~mA} \mathrm{~h} \mathrm{~g}^{-1}\right) .{ }^{176}$ Apart from the above discussed transition metal oxides, improved lithium storage performance has also been achieved when using multishelled hollow structures of $\mathrm{ZnO}, \mathrm{La}_{2} \mathrm{O}_{3}$, and so on. ${ }^{177,178}$
Several main group metal oxides could also be used as anode materials for LIBs. ${ }^{179}$ For instance, Wu et al. intensively investigated a carbon source and Sn salt precursor with regard to the interior and size of multishelled $\mathrm{SnO}_{2}$ hollow spheres. They found that the ratio of glucose to metal precursor plays a critical role. Furthermore, the electrochemical performance of multishelled $\mathrm{SnO}_{2}$ hollow nanospheres was also evaluated when used as an anode for LIBs. Their results demonstrated that the initial discharge and charge capacities were 861 and $442 \mathrm{~mA} \mathrm{~h} \mathrm{~g}^{-1}$, respectively. The considerable irreversible capacity loss indicated an irreversible process in the system, such as electrolyte decomposition or the formation of a solid electrolyte interphase (SEI) layer. With regard to the cycling performance, the charge-discharge capacity remained $214 \mathrm{~mA} \mathrm{~h} \mathrm{~g}^{-1}$ after 100 cycles, demonstrating good capacity retention. In addition, the as-prepared multishelled $\mathrm{SnO}_{2}$ hollow spheres were relatively tolerant to the various charge-discharge currents. The average capacities were $\sim 410,358,298,202$, and $112 \mathrm{~mA} \mathrm{~h} \mathrm{~g}^{-1}$ at current densities of 100,200,500,1000, and $2000 \mathrm{~mA} \mathrm{~g}^{-1}$, respectively. ${ }^{180}$ Zhou et al. synthesized a trilayered microsphere composite of $\mathrm{SiO}_{2} @ \mathrm{SnO}_{2} @ \mathrm{P}(\mathrm{EGDMA}-\mathrm{co}$-MAA) via a combination of Stöber method and in situ coating of $\mathrm{SnO}_{2}$ and P(EGDMA-co-MAA) shells. After the selective removal of the silica core via $\mathrm{HF}$ etching, double-shelled $\mathrm{SnO}_{2} @ \mathrm{P}(\mathrm{EGDMA}-\mathrm{co}$ MAA) hollow microspheres were obtained. Benefiting from the specific hollow structure with a large void space to buffer any 
volume expansion during the alloying and dealloying processes with lithium ions, the as-obtained double-shelled $\mathrm{SnO}_{2}$ @P(EGDMA-co-MAA) hollow microspheres offered a high capacity toward lithium storage. The direct contact of lithium ion and $\mathrm{SnO}_{2}$ component may be effectively avoided since the conductive and flexible P(EGDMA-co-MAA) outer shell as a protective layer was permeable to lithium-ion exchange. Because of the above merits, the obtained $\mathrm{SnO}_{2} @ \mathrm{P}($ EGDMA-co-MAA) sample delivered a high discharge capacity of $1170 \mathrm{~mA} \mathrm{~h} \mathrm{~g}{ }^{-1}$ at $200 \mathrm{~mA} \mathrm{~g}^{-1}$, good rate capacity of $322 \mathrm{~mA} \mathrm{~h} \mathrm{~g}^{-1}$ at $4000 \mathrm{~mA} \mathrm{~g}^{-1}$, and excellent cycling performance with capacity retention of $711.9 \mathrm{~mA} \mathrm{~h} \mathrm{~g}{ }^{-1}$ after 400 cycles at $200 \mathrm{~mA} \mathrm{~g}{ }^{-1} \cdot{ }^{181}$ In order to further increase the lithium storage performance of $\mathrm{SnO}_{2}$, Zhang et al. fabricated heterogeneous $\mathrm{SnO}_{2} @ \mathrm{Fe}_{2} \mathrm{O}_{3}(\mathrm{MOF})$ and $\mathrm{SnO}_{2} @ \mathrm{FeO}_{x}$-C(MOF) hollow multishelled structures by accurately controlling the transformation of MOF casing. When tested as anode materials for LIBs, these heterogeneous hollow multishelled structures demonstrated superior lithium storage capacity and cycling stability as compared to that of the original $\mathrm{SnO}_{2}$ hollow multishelled structures. ${ }^{182}$

Besides single-metal oxides, some ternary metal oxides have also been used as anode materials for LIBs. For example, Li et al. prepared triple-shelled $\mathrm{CuCo}_{2} \mathrm{O}_{4}$ hollow microspheres $\left(\mathrm{T}-\mathrm{CuCO}_{2} \mathrm{O}_{4}\right)$ via a solvothermal/calcination method (Fig. 39a). By controlling the solvothermal time, the number of shells in the final product could be adjusted. When used as an anode for LIBs, T- $\mathrm{CuCo}_{2} \mathrm{O}_{4}$ delivered a higher rate capability and superior cycling stability as compared to those obtained from singleshelled and double-shelled $\mathrm{CuCo}_{2} \mathrm{O}_{4}$ hollow microspheres, as shown in Fig. 39b and c. ${ }^{183}$ Qi et al. demonstrated that multishelled $\mathrm{CoFe}_{2} \mathrm{O}_{4}$ hollow nanospheres delivered a worthwhile lithium storage capacity when compared with those obtained from $\mathrm{CoFe}_{2} \mathrm{O}_{4}$ solid nanospheres and $\mathrm{CoFe}_{2} \mathrm{O}_{4}$ hollow nanospheres with traditional single shells. ${ }^{63}$ Shin et al. used colloidal carbon spheres as the template and prepared multishelled $\mathrm{MgCo}_{2} \mathrm{O}_{4}$ (MCO) hollow microspheres to achieve high rate capability and improved electrochemical stability. Recently, Jiao et al.
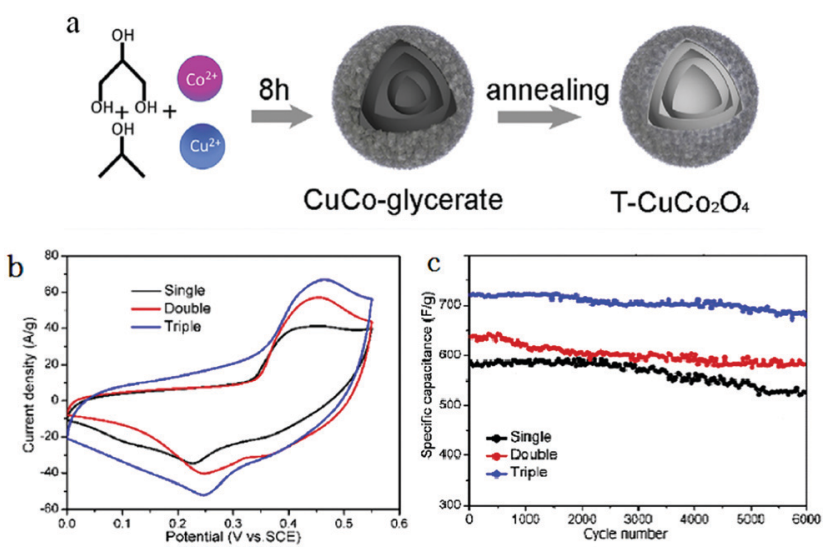

Fig. 39 (a) Schematic illustration of the preparation of $\mathrm{T}-\mathrm{CuCO}_{2} \mathrm{O}_{4}$. (b) $\mathrm{CV}$ curves at $50 \mathrm{mV} \mathrm{s}^{-1}$ and (c) cycling performance of $\mathrm{CuCO}_{2} \mathrm{O}_{4}$ hollow microspheres with different numbers of shells as an anode for LIBs. Reproduced from ref. 183 with permission. prepared triple-shelled $\mathrm{Mn}-\mathrm{Co}-\mathrm{O}$ hollow dodecahedra using manganese-doped zeolitic imidazole framework ZIF-67 as a precursor via continuous two-step calcination. Benefiting from the residual $\mathrm{C}$ and $\mathrm{N}$ and the unique features of this hollow structure, the obtained triple-shelled $\mathrm{Mn}-\mathrm{Co}-\mathrm{O}$ hollow dodecahedra exhibited promising electrochemical performance with highly reversible high rate performance, excellent cycling stability, and high capacity when applied as the cathode in rechargeable alkaline batteries. ${ }^{184} \mathrm{Xu}$ et al. constructed various multishelled $\mathrm{Zn}-\mathrm{Mn}-\mathrm{O}$ hollow microspheres, namely, $\mathrm{ZnMnO}_{3}, \mathrm{ZnMn}_{2} \mathrm{O}_{4}$, and $\mathrm{ZnMn}_{2} \mathrm{O}_{4} / \mathrm{Mn}_{2} \mathrm{O}_{3}$, via a simple programmable heating treatment on a coordination polymer precursor and found that these multishelled hollow microspheres afforded excellent activity and stability when used as the anode for LIBs. ${ }^{185}$

When compared with oxides, metal sulfides, often offering more abundant redox centers and electrochemically active sites, have emerged as a promising electrode candidate for use in rechargeable alkaline batteries. Li et al. designed and synthesized a series of sulfur-rich nickel sulfide multishelled hollow nanospheres, such as $\mathrm{Ni}_{3} \mathrm{~S}_{2}$, NiS, and $\mathrm{NiS}_{2}$, using multishelled NiO hollow spheres as the precursor. These multishelled nickel sulfide hollow spheres possessed excellent electrochemical performance when used as the electrode materials for a rechargeable alkaline battery. ${ }^{186}$

In addition to metallic compounds, carbon materials have also been widely investigated as electrode materials for LIBs. ${ }^{187-190}$ Sun et al. reported the electrochemical performance of multishelled carbon hollow spheres prepared by using silica as the hard template. ${ }^{27}$ Fig. 40a shows the CV curves of quadruple-shelled carbon hollow spheres in the first three cycles at a sweep rate of $0.1 \mathrm{mV} \mathrm{s}^{-1}$. Evidently, the cathodic peak at $\sim 0.5-0.8 \mathrm{~V}$ was observed only in the first cycle, which is related to the formation of a SEI film. The disappearance of this peak after the first cycle indicates a large initial irreversible capability in the subsequent cycles. Fig. 40b shows the charge-discharge profiles of the first three cycles. It has been observed that the voltage drop is very quick initially followed by a plateau at $\sim 0.5-0.8 \mathrm{~V}$ during the first cycle. The plateau could be attributed to the cathodic peak in the CV curves. Based on the charge curve, the first charge capacity was calculated to be $1254 \mathrm{~mA} \mathrm{~h} \mathrm{~g}{ }^{-1}$, which is much higher than that of the other hollow carbon spheres reported elsewhere and nearly 4 times the theoretical value of graphite (372 $\mathrm{mA} \mathrm{h} \mathrm{g}^{-1}$ ). Fig. 40c shows the cycling performance of hollow carbon spheres with different numbers of shells (single, double, and quadruple). Notably, among these three hollow samples, quadruple-shelled carbon spheres show the most promising stability. The specific capacity of quadruple-shelled carbon hollow spheres was retained at $977.6 \mathrm{~mA} \mathrm{~h} \mathrm{~g}{ }^{-1}$ after 40 cycles, while those of single- and double-shelled ones were 303.5 and $712.1 \mathrm{~mA} \mathrm{~h} \mathrm{~g}^{-1}$, respectively. The improved cycling performance of quadruple-shelled carbon hollow spheres is due to the high rate of volume occupation. In addition, the rate capabilities of single- and quadruple-shelled carbon hollow spheres were investigated, as shown in Fig. 40d. Obviously, the capacity of quadruple samples was determined to be $317 \mathrm{~mA} \mathrm{~h}^{-1}$ at a high current density of $800 \mathrm{~mA} \mathrm{~g}^{-1}$, while it was only $85 \mathrm{~mA} \mathrm{~h} \mathrm{~g}^{-1}$ for 

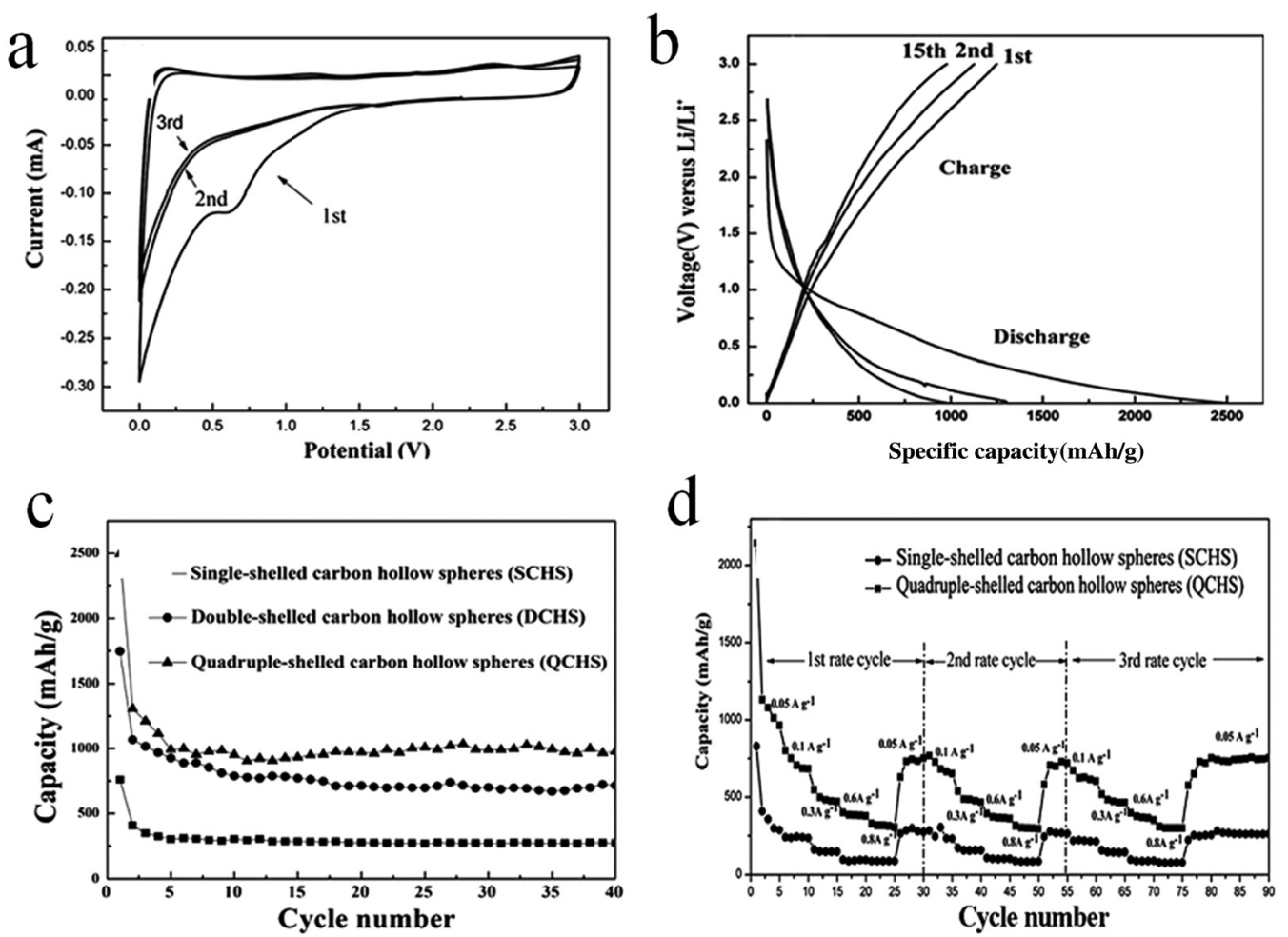

Fig. 40 (a) CV curves of the first 3 cycles and (b) GCD curves at $50 \mathrm{~mA} \mathrm{~g}^{-1}$ for the quadruple-shelled carbon hollow spheres. (c) Cycling performances of carbon hollow spheres for different numbers of shells. (d) Rate capability of quadruple- and single-shelled carbon hollow spheres at various current densities. Reproduced from ref. 27 with permission.

the single-shelled samples. Recently, Xu et al. prepared N-doped double-shelled hollow mesoporous carbon nanospheres, possessing promising electrochemical performance when used as the anode materials for LIBs. ${ }^{80}$ In particular, the sample obtained by carbonization at $800{ }^{\circ} \mathrm{C}$ was demonstrated to offer a discharge specific capacity of $920.3 \mathrm{~mA} \mathrm{~h} \mathrm{~g}{ }^{-1}$ at a current density of $0.1 \mathrm{~A} \mathrm{~g}^{-1}$ after 100 cycles and good rate capability when the current density increased in a step-by-step manner from 0.1 to $5 \mathrm{~A} \mathrm{~g}^{-1}$. The remarkably improved rate capability could be attributed to the increased contact area between the electrode and electrolyte, improved lithium-ion and electron transport, as well as an appropriate amount of $\mathrm{N}$ doping.

Besides anode materials, multishelled hollow structures can also be applied as cathode materials for LIBs. Spinel $\mathrm{LiMn}_{2} \mathrm{O}_{4}$ is one of the most promising alternatives to commercial $\mathrm{LiCoO}_{2}$ when used as the cathode for LIBs because it is environmentally benign, low cost, and highly abundant. Niu et al. presented a facile aerosol ultrasonic spray pyrolysis approach to prepare multishelled $\mathrm{LiMn}_{2} \mathrm{O}_{4}$ hollow nanospheres that were assembled by nanosized $\mathrm{LiMn}_{2} \mathrm{O}_{4}$ particles $(\sim 10-30 \mathrm{~nm})$. The electrochemical properties of these multishelled $\mathrm{LiMn}_{2} \mathrm{O}_{4}$ hollow nanospheres when used as a cathode for LIBs were comprehensively investigated. They found that the capacity of the prepared multishelled $\mathrm{LiMn}_{2} \mathrm{O}_{4}$ hollow nanospheres could reach up to $110 \mathrm{~mA} \mathrm{~h} \mathrm{~g}{ }^{-1}$ (very near to its theoretical value of $148 \mathrm{~mA} \mathrm{~h} \mathrm{~g}^{-1}$ ) with capacity retention of $91.9 \%$ after 400 cycles at a current density of $0.2 \mathrm{~A} \mathrm{~g}^{-1}$. Such excellent properties are directly related to the aggregation of ultrafine $\mathrm{LiMn}_{2} \mathrm{O}_{4}$ particles and the presence of unique mesopores and voids in the sample. ${ }^{191}$ Wang et al. prepared multishelled $\mathrm{LiMn}_{2} \mathrm{O}_{4}$ hollow microspheres using carbonaceous materials as the hard template and systematically investigated their electrochemical performance as a cathode for LIBs. They found that the samples with three shells exhibited relative superior lithium storage capacity as compared to multishelled hollow structures. ${ }^{192}$

Similar to LIBs, sodium-ion batteries have also gained extensive interest in the field of scientific research and engineering applications during the recent years. ${ }^{193-195}$ Recently, metal sulfides/phosphides have also been extensively investigated for applications in rechargeable batteries. Xie et al. prepared multishelled hollow structures of $\mathrm{Sb}_{2} \mathrm{~S}_{3}$ from a MOF template. Owing to the better utilization of the large internal voids, multishelled hollow $\mathrm{Sb}_{2} \mathrm{~S}_{3}$ showed outstanding volumetric energy density and enhanced durability when used as the anode material for sodium-ion batteries. ${ }^{196}$ Huang et al. developed a facile and low-temperature solvothermal process to prepare multishelled $\mathrm{Sn}_{4} \mathrm{P}_{3}$ nanostructures, exhibiting a large specific area and interlayer space. When applied as an anode for a sodium-ion battery, the specific capacity of the as-prepared multishelled $\mathrm{Sn}_{4} \mathrm{P}_{3}$ nanostructures could reach up to $770 \mathrm{~mA} \mathrm{~h} \mathrm{~g}^{-1}$ with capacity retention of $96 \%$ after 50 cycles at a current density of $50 \mathrm{~mA} \mathrm{~g}^{-1} \cdot 197$

Multishelled hollow nanostructures are also advantageous for use in lithium-sulfur batteries. It is well known that the 
loading of sulfur in a cathode is critical for the comprehensive performance of the constructed lithium-sulfur battery. Multishelled hollow nanostructures as sulfur carriers are expected to exhibit the following advantages. First, electrolyte penetration into the cavities may be obviously increased when the shells are porous. Secondly, the thin shells comprising tiny nanoparticles may shorten the charge/mass diffusion paths, resulting in high rate capacity. Finally, the interlayer spaces can buffer the volume expansion during the lithiation/delithiation process. Salhabi et al. employed hollow multishelled $\mathrm{TiO}_{2-x}$ nanospheres as a sulfur carrier that exhibited a high capacity of $903 \mathrm{~mA} \mathrm{~h} \mathrm{~g}^{-1}$ with good capacity retention of $79 \%$ at $0.5 \mathrm{C}$ and impressive cycling stability $(97.5 \%$ over 1000 cycles $) .{ }^{198}$ Chen et al. prepared multishelled CoP hollow nanospheres with high electrical conductivity by calcining the Co(II) complex precursor in the presence of $\mathrm{NaH}_{2} \mathrm{PO}_{2}$. The as-prepared multishelled CoP hollow nanospheres are expected to possess a polar chemisorptive ability toward polysulfides and provide sufficient active sites to trap polysulfides. When applied as the upper current collector in lithium-sulfur batteries, the constructed battery delivers good cycling performance and good rate capability. Significantly, the mass loading of sulfur in such CoP samples can reach up to $3.24 \mathrm{mg} \mathrm{cm}^{-2}$, yielding high capacity and excellent cycling stability. ${ }^{199}$ Chen et al. prepared multishelled carbon hollow nanospheres using an emulsion process. Sulfur could be effectively encapsulated into the multishelled carbon hollow nanosphere by means of an in situ method. The percentage of sulfur in the cathode could reach up to $86 \%$. The prepared multishelled carbon hollow spheres offered enhanced cyclability and good capacity retention when applied as cathodes in lithium-sulfur batteries. ${ }^{200}$

Similar to supercapacitors, the number and thickness of multiple thin shells have a considerable impact on the energy density of alkali metal-ion batteries. Appropriate intershell layer spacing is crucial for buffering volume expansion and short transport pathways facilitate mass transfer. Multishelled hollow nanostructures with a designed composition for each shell show promising prospects for fascinating alkali metal ion and electron transfer, thereby accelerating redox kinetics. Consequently, it is feasible to design multishelled hollow nanostructures as high-performance electrodes to satisfy the increasing demands of energy storage devices.
3.1.3 Dye-sensitized solar cells. Dye-sensitized solar cells, which convert solar energy into electric energy, have been demonstrated to be efficient photovoltaic devices. In order to anchor sufficient dye and harvest a considerable amount of light from sunshine, photoanode materials possessing a high surface area are more preferable. ${ }^{201,202}$ Therefore, wide-bandgap hollow materials, particularly multishelled hollow microspheres made from transition metallic oxides, have emerged as promising candidates for use as photoanodes. ${ }^{203}$ It is well known that $\mathrm{TiO}_{2}$ is a promising semiconductor material that has been widely used in photocatalysis and dye-sensitized solar cells. ${ }^{204-208}$ Hwang et al. developed multishelled porous $\mathrm{TiO}_{2}$ hollow nanoparticles (MS $\mathrm{TiO}_{2}$ HNPs) as a photoanode for use in dye-sensitized solar cells. ${ }^{22}$ They found that $\mathrm{MS} \mathrm{TiO}_{2}$ HNPs exhibited a large specific surface area of $171.3 \mathrm{~m}^{2} \mathrm{~g}^{-1}$, nearly 2.7 times that of single-shelled $\mathrm{TiO}_{2}$ hollow nanoparticles (SS TiO 2 HNPs) $\left(63.6 \mathrm{~m}^{2} \mathrm{~g}^{-1}\right)$ and 1.33 times that double-shelled $\mathrm{TiO}_{2}$ hollow nanoparticles (DS $\mathrm{TiO}_{2}$ HNPs) $\left(128.4 \mathrm{~m}^{2} \mathrm{~g}^{-1}\right)$. Such a large surface area facilitates the adsorption of organic dyes in dye-sensitized solar cells. Meanwhile, the diffuse reflectance of $\mathrm{MS} \mathrm{TiO}_{2} \mathrm{HNPs}$ could be measured to be more effective than those of $\mathrm{SS} \mathrm{TiO}_{2}$ HNPs and DS $\mathrm{TiO}_{2}$ HNPs (Fig. 41a) owing to enhanced light scattering. Once employed as a photoanode for dye-sensitized solar cells, $\mathrm{MS} \mathrm{TiO}_{2}$ HNPs also possess superior performance in terms of current density-voltage characteristics and incident photon-to-electron conversion efficiencies (IPCEs) as compared to those obtainable from SS $\mathrm{TiO}_{2}$ HNPs and DS $\mathrm{TiO}_{2} \mathrm{HNPS}$ (Fig. 41b and c). Wu et al. reported that shell-in-shell $\mathrm{TiO}_{2}$ hollow spheres, prepared by hydrothermal process and postheating treatment, possess excellent light-scattering properties and deliver overall conversion efficiency of $9.10 \%$, which is higher than that of a pristine P25 photoanode (7.65\%). ${ }^{209}$

Besides $\mathrm{TiO}_{2}$, $\mathrm{ZnO}$ has also been regarded as a promising photoanode candidate for use in dye-sensitized solar cells. $^{210-212}$ Tian et al. tested the performance of multishelled ZnO hollow microspheres for this purpose, which was obtained by the hydrothermal treatment of a zinc gluconate solution with the desired acidity followed by a calcination treatment. ${ }^{44}$ Cells based on multishelled $\mathrm{ZnO}$ hollow microspheres deliver a higher current density of $13.04 \mathrm{~mA} \mathrm{~cm} \mathrm{~cm}^{-2}$ with conversion efficiency of $4.13 \%$, which is much better than the cells based on commercial $\mathrm{ZnO}$ solid spheres. The improved photoanode
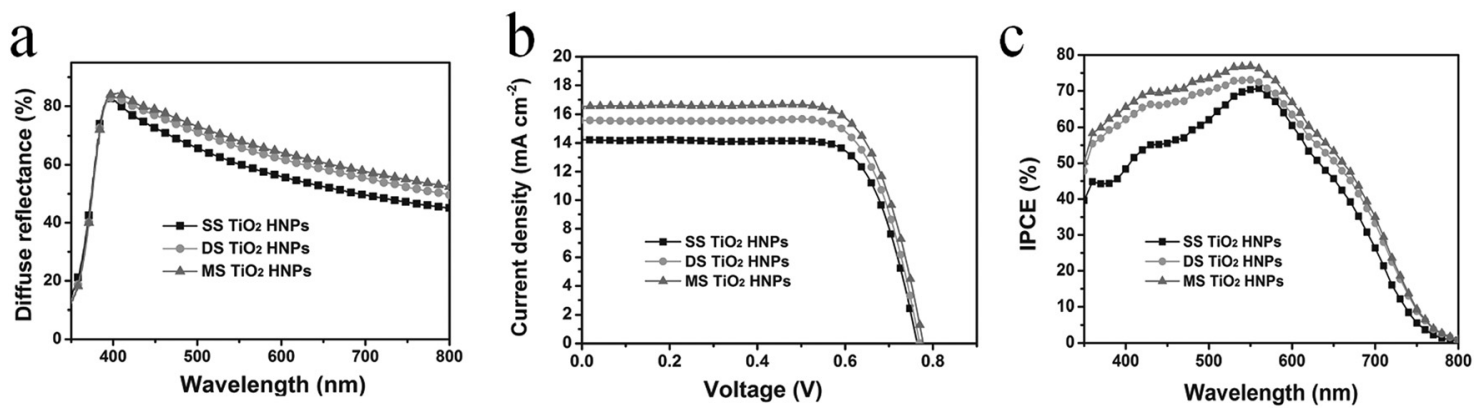

Fig. 41 (a) Diffuse reflectance spectra, (b) current density-voltage characteristics (J-V curves), and (c) IPCEs of SS-TiO $-\mathrm{HNPs}_{2}$ (square), DS-TiO $2-\mathrm{HNPs}$ (circle), and $\mathrm{MS}-\mathrm{TiO}_{2}-\mathrm{HNPs}$ (triangle). Reprinted from ref. 22 with kind permission. 
performance of the as-prepared multishelled $\mathrm{ZnO}$ hollow microspheres is undoubtedly related to the features of a multishelled structure, where the large surface area can load abundant dye molecules and relatively large size can scatter the incident light.

In another study, Wang et al. demonstrated that quadrupleshelled ZnO hollow microspheres could deliver conversion efficiency of $5.60 \%$, which is an increase of $20 \%$ of the overall conversion efficiency as compared to that obtained from a standard solid-particle-shaped $\mathrm{ZnO}$ photoanode. They also noted that the thickness of the exterior shells had a significant impact on the light-harvesting capability and the generated photocurrent. Typically, a thicker exterior shell may scatter more light and afford a higher photocurrent. Besides $\mathrm{ZnO}$, they also investigated the performance of multishelled $\mathrm{SnO}_{2}$ hollow microspheres as a photoanode for use in dye-sensitized solar cells. $^{34,38}$

$\mathrm{CeO}_{2}$, a rare-earth oxide with a bandgap of $3.2 \mathrm{eV}$, has also been suggested as a promising photoanode material for use in solar cells. Umable et al. synthesized $\mathrm{CeO}_{2}$ nanoparticles using a solution combustion process. The characteristic of spongy and porous morphology rendered them with high incident photon current efficiency of $68 \% .^{213}$ Nevertheless, $\mathrm{CeO}_{2}$ nanoparticles often suffer from the tradeoff between the scattering effect and loss of incident light owing to the transmission of light. In order to overcome this issue, double-shelled $\mathrm{CeO}_{2}$ @ $\mathrm{TiO}_{2}$ hollow sphere composites were proposed by Diao's group, which were prepared by a one-pot hydrothermal method followed by coating with a $\mathrm{TiO}_{2}$ shell. ${ }^{214}$ They found that the dye-loading capacity could be improved owing to the doubleshelled $\mathrm{CeO}_{2}$ hollow sphere core; therefore, the hybrid doubleshelled $\mathrm{CeO}_{2} @ \mathrm{TiO}_{2}$ hollow sphere exhibited higher conversion efficiency $(7.95 \%)$ than that of bare $\mathrm{TiO}_{2}$ electrodes $(5.72 \%)$. In addition, Diao's group also did a series of works on $\mathrm{CeO}_{2}$ based photoanode materials for use in dye-sensitized solar cells, namely, $\mathrm{CeO}_{2}: \mathrm{Yb}, \mathrm{Er} @ \mathrm{SiO}_{2} @ \mathrm{Ag}$ upconversion composite; ${ }^{215}$ $\mathrm{Er}, \mathrm{Yb}-\mathrm{CeO}_{2}$ hollow spheres; ${ }^{216}$ and $\mathrm{Yb}$, Er-doped $\mathrm{CeO}_{2}$ nanotubes. $^{217}$

Sunlight absorption and dye loading are two crucial factors that determine the performance of dye-sensitized solar cells. The number of shells and intershell spacing are the two most important characteristics of multishelled hollow nanostructures.
Typically, larger number of shells and smaller intershell spacing are expected to offer superior sunlight-harvesting capability and higher energy conversion efficiency. The porosity of shells as well as grain size of multishelled hollow nanostructures play a significant role in influencing the loading capacity of dyes. Normally, shells composed of smaller grains can provide a larger surface area to accommodate dyes. Meanwhile, porous shells can yield multiple benefits. Firstly, they can provide a larger specific surface area for dye loading. Lastly, porous shells are expected to improve the lightabsorbing efficiency by facilitating multiple sunlight reflections and scattering between the shells.

3.1.4 Catalytic hydrogen evolution/water splitting. Besides converting and storing unstable but renewable energy, exploring clean energy sources is also critical to realize sustainable development. Hydrogen is considered to be the most promising clean energy source since the combustion product of hydrogen is $\mathrm{H}_{2} \mathrm{O}$, having nearly no harm to society or environment. It is well known that the purity of $\mathrm{H}_{2}$ is vital for its storage and applications since it is easily combustible when mixed with air $/ \mathrm{O}_{2}$ above a critical temperature. Electrocatalytic hydrogen evolution is regarded as one of the most promising strategies to obtain $\mathrm{H}_{2}$ with ultrahigh purity, which is important for storage and transformation, as well as the final applications. Waqas et al. investigated the light-driven water oxidation performance of multishelled $\mathrm{TiO}_{2} / \mathrm{Fe}_{2} \mathrm{TiO}_{5}$ heterostructured hollow microspheres. ${ }^{218}$

Qi et al. reported a self-template route to synthesize tripleshelled $\mathrm{CeO}_{2}$ hollow microspheres, involving hydrothermally treating the mixed aqueous solution containing glucose, $\mathrm{CeCl}_{3}$ precursors and urea, as well as a subsequent calcination treatment, as shown in Fig. 42a. ${ }^{219}$ The following chemical processes are expected to occur in this system. Firstly, glucose is converted into carbonaceous microspheres under the hydrothermal condition, which may act as a template to take up $\mathrm{Ce}^{3+}$ through electrostatic attractions. The main role of urea is to deprotonate the functional groups inside the carbonaceous microspheres, thereby enabling interactions between the carbonaceous material and $\mathrm{Ce}^{3+}$. Finally, the carbonaceous microspheres containing $\mathrm{Ce}^{3+}$ can generate triple-shelled $\mathrm{CeO}_{2}$ hollow spheres by calcination in air. In this process, the carbonaceous microsphere is decomposed into gases and $\mathrm{Ce}^{3+}$ gets oxidized into $\mathrm{CeO}_{2}$. Impressively, the morphology and
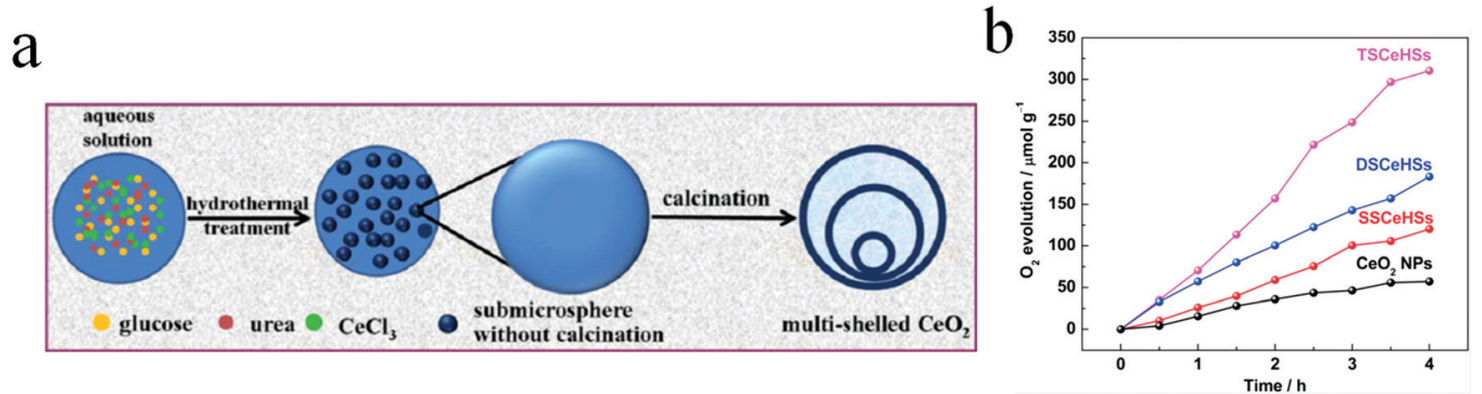

Fig. 42 (a) Proposed formation process of multishelled $\mathrm{CeO}_{2}$ hollow microspheres. (b) Comparison of photocatalytic oxygen evolution performance of $\mathrm{CeO}_{2} \mathrm{NPs}$ and as-prepared multishelled $\mathrm{CeO}_{2}$ hollow microspheres with different numbers of shells. Reproduced from ref. 219 with kind permission. 
structure of the final product can vary with the experimental parameters, including the dosage of each reactant, crystalline reaction, and calcination temperature. Furthermore, the results of the four $\mathrm{CeO}_{2}$ samples, namely, commercial $\mathrm{CeO}_{2}$ nanoparticles $\left(\mathrm{CeO}_{2}\right.$ NPs $)$, single-shelled $\mathrm{CeO}_{2}$ hollow spheres (SSCeHSs), double-shelled $\mathrm{CeO}_{2}$ hollow spheres (DSCeHSs), and triple-shelled $\mathrm{CeO}_{2}$ hollow spheres (TSCeHSs), are shown in Fig. 42b. Evidently, all the hollow $\mathrm{CeO}_{2}$ spheres are superior over $\mathrm{CeO}_{2} \mathrm{NPs}$ with regard to catalytic activity. Among the three hollow samples, TSCeHSs exhibit the best performance. The average oxygen evolution rate from the oxidation of water was $78 \mu \mathrm{mol}\left(\mathrm{g}_{\text {cat }} \mathrm{h}\right)^{-1}$. Such a remarkably improved photocatalytic activity of TSCeHSs was directly associated with the considerably increased surface area and number of active sites generated by multishelled features, improving the separation of electron-hole pairs.

Wei et al. prepared $\mathrm{SrTiO}_{3}-\mathrm{TiO}_{2}$ heterogeneous hollow multishelled structures (STHoMs) by the hydrothermal crystallization of $\mathrm{SrTiO}_{3}$ on the surface of $\mathrm{TiO}_{2}$ hollow multishelled structures, resulted in outstanding solar water-splitting performance of $10.6 \mathrm{mmol} \mathrm{h}^{-1}$ for $\mathrm{H}_{2}$ production and $5.1 \mathrm{mmol} \mathrm{h}^{-1}$ for oxygen evolution. They explained that the synergistic effect of $\mathrm{SrTiO}_{3}$ and $\mathrm{TiO}_{2}$ afforded better separation efficiency of the photogenerated charge carriers and hollow multishelled structures increased the light-absorption ability of the $\mathrm{SrTiO}_{3}-$ $\mathrm{TiO}_{2}$ heterogeneous catalyst. ${ }^{20}$

\subsection{Environmental-remediation-related applications}

3.2.1 Gas sensors. Gas sensors are devices capable of detecting the presence as well as the exact concentration of the target gaseous species, such as explosives, toxic gases, or volatile organic compounds (VOCs), in space- and time-confined environments. Nowadays, gas sensors play an increasingly important role in indoor safety, environment monitoring, human health, and automotive applications. Currently, gas sensors based on metal oxide semiconductors are among the most widely utilized tools to detect various targeted gases owing to their advantages such as being small, portable, and inexpensive. However, in order to achieve optimal sensing performances, these sensors usually need high working temperatures. For multishelled hollow nanostructures, their major advantage is the high specific area, which is expected to provide a larger number of adsorption centers on the surface of the sensor layer as compared to their traditional solid counterparts. Consequently, the rates of surface reactions can be increased and consequently improve the sensing property.

Wang et al. prepared multishelled ZnO core-shell hollow microspheres with a diameter of $\sim 0.4-3.5 \mu \mathrm{m}$ by using carbonaceous materials as the hard template. A chemical gas sensor based on the as-prepared multishelled $\mathrm{ZnO}$ core-shell hollow microspheres exhibited rapid, high, and selective response toward toluene. ${ }^{221}$ At the optimal operating temperature of $300{ }^{\circ} \mathrm{C}$, the response and recovery times of the sensor for toluene at $20 \mathrm{ppm}$ were 0.3 and $3 \mathrm{~s}$, respectively. The detection limit was as low as $1 \mathrm{ppm}$ and the detectable concentration range was up to $2000 \mathrm{ppm}$. Qu et al. synthesized multishelled mixed Ni-Co oxide microspheres, which showed substantial selectivity and remarkable sensitivity $\left(11.5-5 \mathrm{ppm}\right.$ at $255^{\circ} \mathrm{C}$ ) toward xylene. Significantly, the selectivity and response toward xylene could be maintained with excellent humidity resistance, suggesting the potential of such multishelled $\mathrm{Ni}-\mathrm{Co}$ oxide microspheres in the detection of ground-level xylene gas in the environment. $^{222}$

With the development of industries and widespread utilization of vehicles, the increasing consumption of fossil fuels has generated a large amount of $\mathrm{NO}_{x}\left(\mathrm{NO}\right.$ and $\left.\mathrm{NO}_{2}\right)$, which is harmful to the domestic environment as well as the human body. The accurate content of $\mathrm{NO}_{2}$ in air even at a very low concentration is an important parameter in order to evaluate air quality. Kim et al. developed a highly sensitive and selective $\mathrm{NO}_{2}$ sensor based on multishelled $\mathrm{WO}_{3}$ microspheres. ${ }^{223}$ As shown in Fig. 43, the number of shells of the $\mathrm{WO}_{3}$ hollow microspheres has a significant impact on the sensor performance. Such detection ability can be attributed to the enhanced gas accessibility of the yolk-shell morphology with thin and permeable multiple shells.

As a commonly applied organic solvent in the industry and research laboratory, acetone is characterized as a volatile and deleterious chemical. If the acetone content in the environment reaches up to $450 \mathrm{mg} \mathrm{m}^{-3}$, the health of human beings can be considerably threatened. Meanwhile, as reported by medical research studies, acetone concentration can be a signal for diabetes diagnoses since the acetone concentration exhaled from a healthy person is usually lower than $0.8 \mathrm{ppm}$, while it equals to/exceeds $1.8 \mathrm{ppm}$ for diabetic patients. Therefore, developing sensitive and selective acetone sensors to detect acetone concentration in the public or human body is worthwhile. Li et al. developed an acetone gas sensor with high
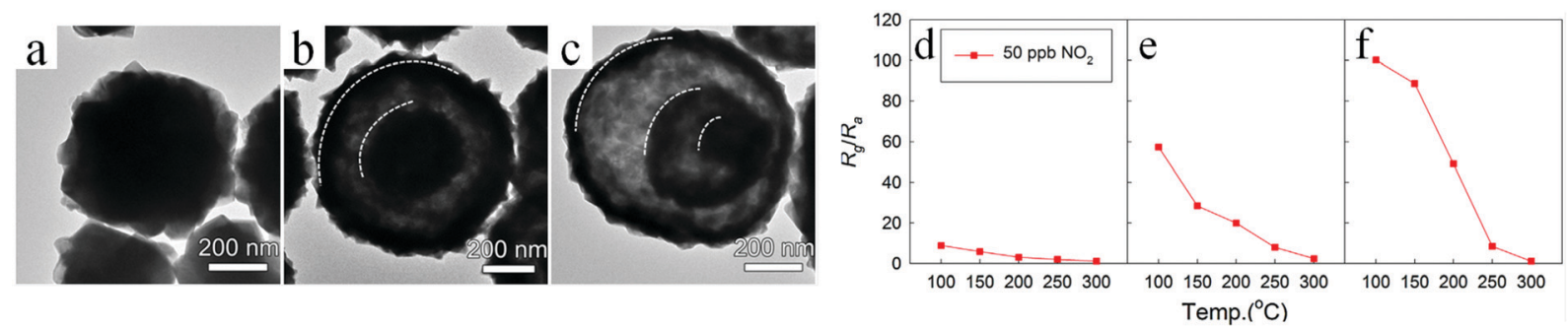

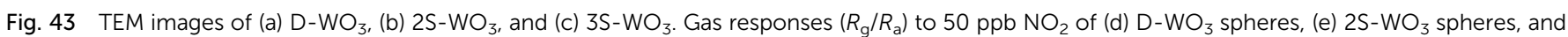
(f) $3 \mathrm{~S}-\mathrm{WO}_{3}$ spheres. Reprinted from ref. 223 with kind permission. 
sensitivity and selectivity based on double-shelled $\mathrm{ZnO}$ hollow microspheres. ${ }^{24}$ This device yielded high response toward $100 \mathrm{ppm}$ acetone (101.1), achieving a rapid response rate and recovery process (within $1 / 7 \mathrm{~s}$ ). Moreover, the substantially low detection limit $(0.5 \mathrm{ppm})$, low operating temperature $\left(40{ }^{\circ} \mathrm{C}\right)$, high selectivity, and long-term stability showed that the doubleshelled $\mathrm{ZnO}$ hollow microspheres are a reliable solution to the problem of acetone detection.

Since the sensing mechanism depends on the interaction between the target gas and surface of the sensing layer, the sensibility of metal oxide gas sensors relies on the available sensing surface and target gas concentration. It is well known that the different facets of nanomaterials may show different affinities toward certain gases. Recently, Zheng et al. reported $\mathrm{Y}_{2} \mathrm{O}_{3}$ multishelled hollow structures with exposed $\{220\}$ facets, which makes a significant contribution to the detection of methanol with ultrahigh sensitivity and selectivity. ${ }^{225}$

It has been reported that the performance of semiconductorbased gas sensors may be further enhanced by loading uniform noble metal nanoparticles. ${ }^{226-228}$ Yoon and co-workers prepared Pd-loaded quintuple-shelled $\mathrm{Co}_{3} \mathrm{O}_{4}$ microspheres via spray drying followed by thermal treatment. ${ }^{229}$ These Pd-loaded quintuple-shelled $\mathrm{Co}_{3} \mathrm{O}_{4}$ microspheres exhibited unprecedented response (resistance ratio) and selectivity toward toluene and $p$-xylene against various interfering gases such as ethanol, benzene, formaldehyde, ammonia, nitrogen monoxide, carbon monoxide, and hydrogen. They also compared their performance with $\mathrm{Pd}-\mathrm{CO}_{3} \mathrm{O}_{4}$ nanoparticles, confirming that multishelled $\mathrm{Pd}-\mathrm{Co}_{3} \mathrm{O}_{4}$ is much superior to other materials. The excellent gas sensing performance could be attributed to several aspects. On one hand, the serious agglomeration characteristics of the $\mathrm{Pd}-\mathrm{Co}_{3} \mathrm{O}_{4}$ nanoparticles yield absolute results in lowering the gas response. For multishelled $\mathrm{Pd}-\mathrm{Co}_{3} \mathrm{O}_{4}$ microspheres, the outermost shell may be the determining factor that affects the gas response. On the other hand, it is the effective dissociation of methylbenzenes into active small species that can promote the selectivity of the fabricated sensors. Undoubtedly, loading metallic nanoparticles can improve the catalytic activity of oxide semiconductors. Meanwhile, multishelled structures may favor the dissociation of methylbenzene by providing more active sites and large space as compared to those afforded by solid nanoparticles. Apart from the above study, Sun et al. demonstrated that PdO-functionalized double-shelled $\mathrm{Fe}_{2} \mathrm{O}_{3}$ hollow nanospheres exhibit superior sensing properties toward acetone owing to the larger surface area and high catalytic activity of PdO. ${ }^{230}$ Ma et al. recently demonstrated that $\mathrm{Au}$ nanoparticles could also further improve the sensor's response of $\alpha-\mathrm{Fe}_{2} \mathrm{O}_{3}$ double-shelled hollow spheres owing to the catalytic sensitization from Au nanoparticles. ${ }^{231}$

The sensing selectivity, sensitivity, and stability are considerably dependent on the interaction between the detected molecules and multishelled hollow nanostructures. Both porosity and hydrophilic characteristics affect the substrate with suitable size/configuration and specific groups accessing the interior of the multishelled hollow nanostructures, leading to worthwhile sensing selectivity. Multiple thin shells often provide abundant responsive sites and the transportation of detected molecules may be facilitated if the shells are porous owing to the capillary effect. In addition, the stability of sensors based on a higher number of shells is expected to be better than those having fewer shells owing to the supporting effect of multiple shells.

3.2.2 Catalytic degradation of pollutants. Environmental issues are becoming increasingly serious day by day, which has promoted the development of pollutant treatment techniques. Catalytic degradation/reduction, as a green route and safe operating technology, has been demonstrated to be one of the most promising solutions to this enhanced environmental crisis. Advanced materials with high catalytic performances have always been the goal of scientists for prominent applicability in pollutant elimination. Ma et al. prepared hierarchically porous $\mathrm{ZnO}$ hollow spheres with controllable number of shells using an unusual temperature gradient crystallization route and the photocatalytic degradation of rhodamine B over the as-prepared $\mathrm{ZnO}$ samples. ${ }^{232}$ For hierarchical $\mathrm{ZnO}$ porous hollow spheres, the number of shells is dependent on the photocatalytic activity toward rhodamine B decomposition. In particular, the catalytic activity increased with the number of shells progressively increasing from one to three. In addition, triple-shelled porous $\mathrm{ZnO}$ hollow samples lose only $7 \%$ of their initial activity after 5 cycles. The progressively enhanced activity by increasing the number of shells to multishelled hollow structures with additional interior voids allow multiple reflections of UV light; hence, higher utilization efficiency of the light source can be assured. Hu et al. prepared double-shelled $\alpha-\mathrm{Fe}_{2} \mathrm{O}_{3}$ hollow spheres using the solvothermal route with the assistance of a carbonaceous template. The obtained double-shelled $\alpha-\mathrm{Fe}_{2} \mathrm{O}_{3}$ hollow spheres could efficiently catalyze visible-light degradation. Zhang et al. reported the dual-functional catalytic performance of multishelled $\mathrm{FeCo}_{2} \mathrm{O}_{4}$ hollow microspheres supported on cotton cellulose fibers (CCFs) toward the photodegradation of tetracycline (TC) and malachite green (MG), as well as the catalytic reduction of 4-nitrophenol (4-NP) ${ }^{233}$ On the basis of the Mott-Schottky plots, active species analysis, and UV-vis experiments, the active species during the photocatalytic oxidation processes are mainly produced by $\mathrm{O}_{2}^{-}$, which can efficiently inhibit the recombination of photogenerated carriers and promote the photocatalytic degradation of pollutants. With regard to the catalytic reduction of 4-NP, benefiting from the particular 3d electronic configurations (elemental $\mathrm{Fe}$ and $\mathrm{Co}$ ), $\mathrm{FeCO}_{2} \mathrm{O}_{4}$ can induce the catalytic reduction by giving electrons from $\mathrm{BH}_{4}{ }^{-}$-an electron donor. Once the 4-NP ions were absorbed onto the surface of $\mathrm{CCFs}, \mathrm{BH}_{4}{ }^{-}$reacted with the $\mathrm{FeCO}_{2} \mathrm{O}_{4}$ component in the composite, yielding hydrogen species and electrons. As a consequence, the $-\mathrm{NO}_{2}$ group in 4-NP was reduced to the $-\mathrm{NH}_{2}$ group. The excellent catalytic performance was directly related to the following aspects. First, the particular hollow multishelled structures supplied multiple catalytic active sites and assured the utilization efficiency of visible light, allowing for increased multiple reflections of incident light. Furthermore, the huge specific surface area could accommodate more reactants, leading to improved catalytic performance. 


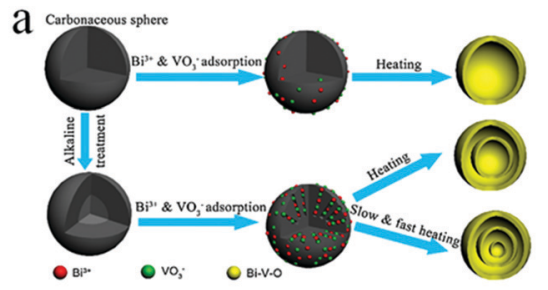

b
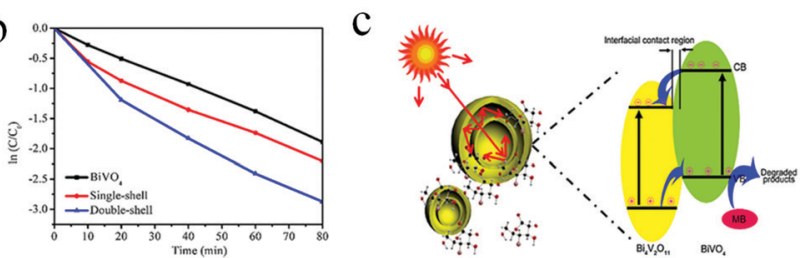

Fig. 44 (a) Schematic illustration of the synthesis procedure of multishelled $\mathrm{Bi}-\mathrm{V}-\mathrm{O}$ hollow microspheres. (b) Catalytic performance of solid $\mathrm{BiVO}_{4}$ and single- and double-shelled $\mathrm{Bi}-\mathrm{V}-\mathrm{O}$ hollow microspheres. (c) Schematic diagram of the photocatalytic mechanism of the heterostructures of multishelled $\mathrm{Bi}-\mathrm{V}-\mathrm{O}$ hollow microspheres. Reprinted from ref. 234 with kind permission.

When compared with simple metal oxides, bimetal oxides usually exhibit superior photocatalytic performance. Zong et al. designed a modified carbonaceous microsphere template method to prepare multishelled hollow spheres of heterostructured $\mathrm{BiVO}_{4}$ (Fig. 44a). The multishelled heterostructured $\mathrm{BiVO}_{4}$ hollow spheres exhibited high photocatalytic activity toward the degradation of methylene blue under visible light, and the double-shell spheres with the highest $\mathrm{Bi}_{4} \mathrm{~V}_{2} \mathrm{O}_{11}$ content delivered the highest photocatalytic activity (Fig. 44b). The excellent photocatalytic performance could be attributed to the effective utilization of visible light induced by multiple reflections of their special multishelled hollow spheres, as shown in the schematic in Fig. 44c. ${ }^{234}$ By taking full advantage of this concept, the excellent photocatalytic degradation of organic pollutants could also be achieved using multishelled $\mathrm{Bi}_{2} \mathrm{WO}_{6}$ hollow microspheres. ${ }^{235}$

Liu et al. demonstrated that double-shelled $\mathrm{ZnFe}_{2} \mathrm{O}_{4}$ hollow microspheres exhibited excellent photocatalytic activity toward the degradation of gaseous $o$-dichlorobenzene $\left(o\right.$-DCB). ${ }^{236}$ The degradation ratio could reach up to $74 \%$ within $7 \mathrm{~h}$, which is higher than that of solid spheres (51\%) and yolk-shell spheres (65\%). Moreover, the double-shelled $\mathrm{ZnFe}_{2} \mathrm{O}_{4}$ hollow microsphere catalyst possesses high stability (the degradation ratio retaining $70 \%$ after 5 repetitions). Such outstanding photocatalytic performance could be attributed to the advantages of double-shelled hollow structures in the adsorption of reactants and sunlight-harvesting capability.

Fig. 45a shows the diffusion characteristics of gaseous $o$-DCB over solid spheres and yolk-shell and double-shelled spheres. Notably, the diffusion rate of $\mathrm{ZnFe}_{2} \mathrm{O}_{4}$ double-shelled spheres is much higher than the rates for the other structures during the adsorption period. This can be directly attributed to the fact that double-shelled hollow spheres can provide a higher surface area and more intricate pore structures than a

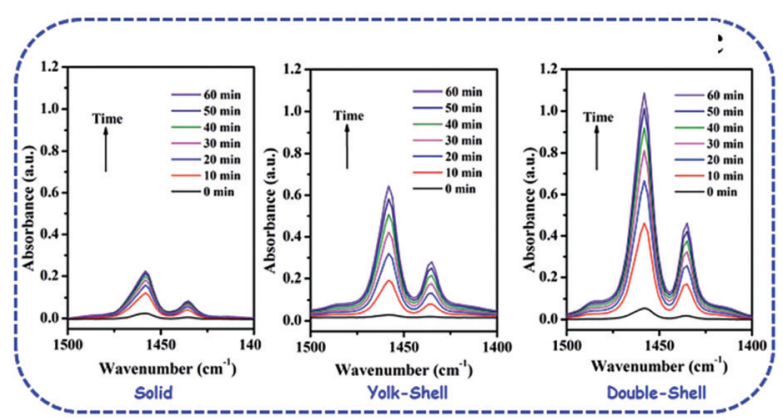

b

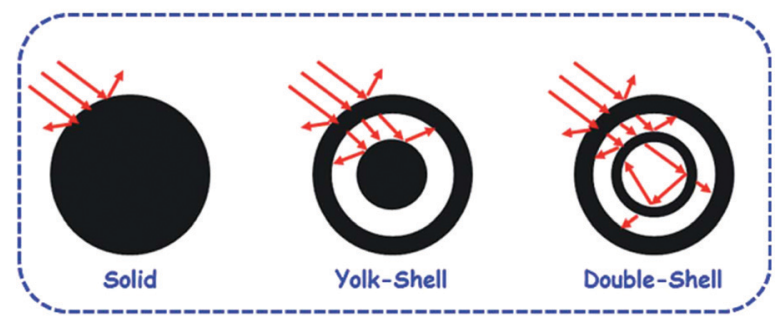

Fig. 45 Comparison of the diffusion characteristics of o-DCB (a) and light diffusion (b) over solid, yolk-shell, and double-shelled $\mathrm{ZnFe}_{2} \mathrm{O}_{4}$ spheres. Reprinted from ref. 236 with permission.

those provided by solid and yolk-shell spheres. Importantly, the superior photocatalytic performance of double-shelled structures is also likely to enhance the effective light absorption for multiple scatterings, as shown in Fig. 45b.

Besides the catalytic degradation of organic pollutants in water, multishelled hollow nanostructures also show promising potential in the catalytic reduction of gas pollutants, such as $\mathrm{NO}, \mathrm{CO}$, and $\mathrm{CO}_{2}$. For example, Ma et al. prepared triple-shelled $\mathrm{CeO}_{2}-\mathrm{MnO}_{x}$ hollow hybrid spheres by using a carbonaceous microsphere as the sacrificial hard template. The as-prepared triple-shelled $\mathrm{CeO}_{2}-\mathrm{MnO}_{x}$ hollow spheres showed apparent advantages when compared with traditional $\mathrm{CeO}_{2}-\mathrm{MnO}_{x}$ nanoparticles, as well as single-shell and double-shell hollow spheres, when applied as a catalyst for the selective catalytic reduction of $\mathrm{NO}$ with $\mathrm{NH}_{3} \cdot{ }^{237}$ As shown in Fig. 46a, the catalytic performances of the samples can be ranked in the following order: triple-shelled $>$ double-shelled $>$ single-shelled $>$ nanoparticles. In particular, triple-shelled $\mathrm{CeO}_{2}-\mathrm{MnO}_{x}$ hollow spheres yielded the sustained conversion of $100 \%$ in the temperature range of $150-250{ }^{\circ} \mathrm{C}$. Such superior catalytic performance of triple-shelled $\mathrm{CeO}_{2}-\mathrm{MnO}_{x}$ hollow spheres can be attributed to the synergistic effect between elemental Ce and Mn. Furthermore, the water resistance performance of these catalysts was also investigated. As shown in Fig. 46b, the addition of water into a mixture of the reactant gases can decrease the conversion of NO for all the catalysts. In particular, the NO conversions of single-, double-, and triple-shelled $\mathrm{CeO}_{2}-$ $\mathrm{MnO}_{2}$ hollow catalyst decreased from their initial conversion to about $70 \%$ in the first hour and then maintained the conversion well up to $14 \mathrm{~h}$ in the presence of water when the reaction temperature was set at $150{ }^{\circ} \mathrm{C}$. However, a decrease from $80 \%$ to below $40 \%$ of the NO conversion was observed for the $\mathrm{CeO}_{2}-\mathrm{MnO}_{2}$ nanoparticle catalyst. The deactivation of the $\mathrm{CeO}_{2}-\mathrm{MnO}_{2}$ catalyst 

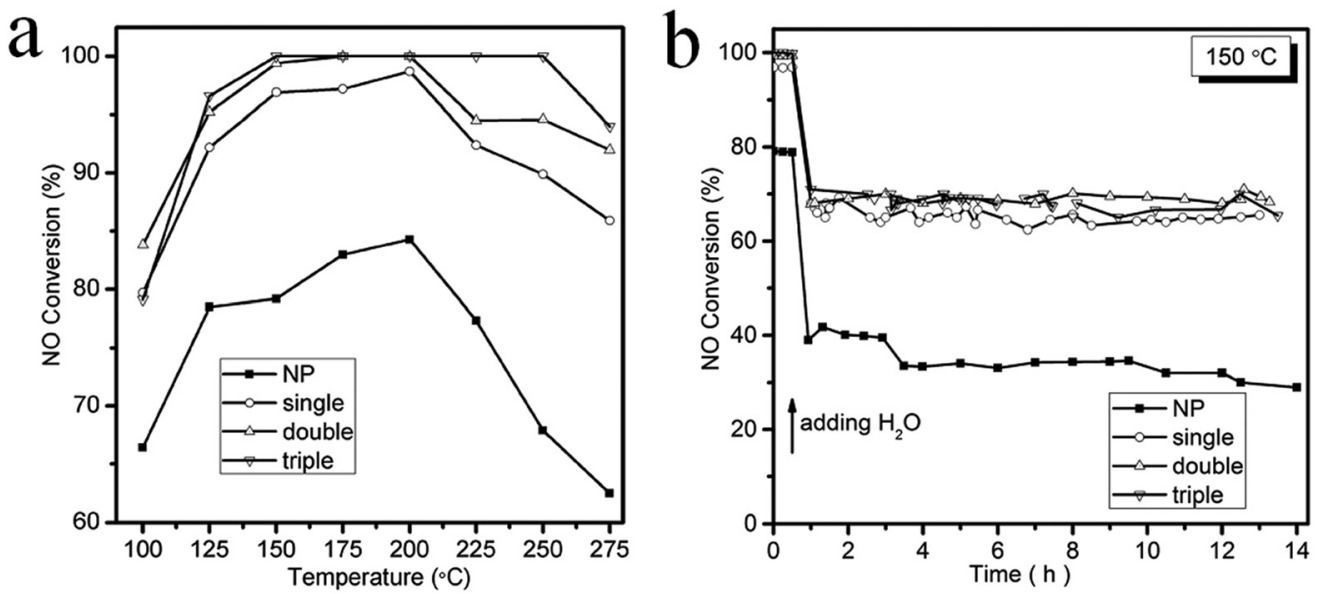

Fig. $46 \mathrm{NO}$ conversion of the $\mathrm{CeO}_{2}-\mathrm{MnO}_{x}$ catalysts as a function of temperature in the absence of water (a) and in the presence of water at $150{ }^{\circ} \mathrm{C}$ (b). Reproduced from ref. 237 with kind permission.

may be related to the formation of hydroxyls on the surface of the catalyst owing to the adsorption of $\mathrm{H}_{2} \mathrm{O}$, leading to a decline in the activity. A typical example is given by Wang et al. They prepared hollow multishelled $\mathrm{Co}_{3} \mathrm{O}_{4}-\mathrm{CeO}_{2-x}$ composites via a general strategy using the sequential template approach with a facile and efficient electrostatic spray process. When compared with the simple components of $\mathrm{Co}_{3} \mathrm{O}_{4}, \mathrm{CeO}_{2}$, and heterogeneous $\mathrm{Co}_{3} \mathrm{O}_{4}-\mathrm{CeO}_{2-x}$ nanoparticles, the multishelled hollow $\mathrm{Co}_{3} \mathrm{O}_{4}-\mathrm{CeO}_{2-x}$ composites exhibited much superior catalytic activity toward the oxidation of $\mathrm{CO}$ (complete conversion temperature is $\left.166.9^{\circ} \mathrm{C}\right)$ and stability $(100 \mathrm{~h})$. They claimed that the worthwhile catalytic performance can be attributed to two factors. For one thing, the synergistic effects generated by the combination of $\mathrm{Co}_{3} \mathrm{O}_{4}$ and $\mathrm{CeO}_{2-x}$ facilitate the activation of oxygen. For another thing, abundant active sites and gas transformation channels of such multishelled hollow structures facilitate gas diffusion and maintain structural stability. ${ }^{238}$

Nowadays, the excessive emissions of $\mathrm{CO}_{2}$ from the accelerated consumption of fossil fuels have increased the threat to the climate. Therefore, the transformation of $\mathrm{CO}_{2}$ has attracted extensive attention. Multishelled hollow nanostructures show promising potential in the photocatalytic reduction of $\mathrm{CO}_{2}$. Recently, Wang and co-workers developed multishelled $\mathrm{Co}_{3} \mathrm{O}_{4}$ hollow dodecahedra using a sequential template approach by adopting MOFs as the template. ${ }^{239} \mathrm{Co}_{3} \mathrm{O}_{4}$ nanocrystals with multishelled hollow structures were assembled in the desired orientation, forming a unique shell with the dominant exposure of the (111) facets. It was observed that the catalytic activity of quadruple-shelled $\mathrm{Co}_{3} \mathrm{O}_{4}$ hollow dodecahedra was superior over those obtained from $\mathrm{Co}_{3} \mathrm{O}_{4}$ nanoparticles and $\mathrm{Co}_{3} \mathrm{O}_{4}$ hollow structures without the controlled facet in $\mathrm{CO}_{2}$ photoreduction.

3.2.3 Adsorptive removal of pollutants. Wang et al. prepared multishelled $\mathrm{Co}_{3} \mathrm{O}_{4}-\mathrm{Fe}_{3} \mathrm{O}_{4}$ hollow spheres (M-MHs) via a simple hydrothermal method without using any hard/soft template. ${ }^{240}$ As shown in Fig. 47a-c, the number of shells of the as-prepared M-MHs could be effectively controlled, including
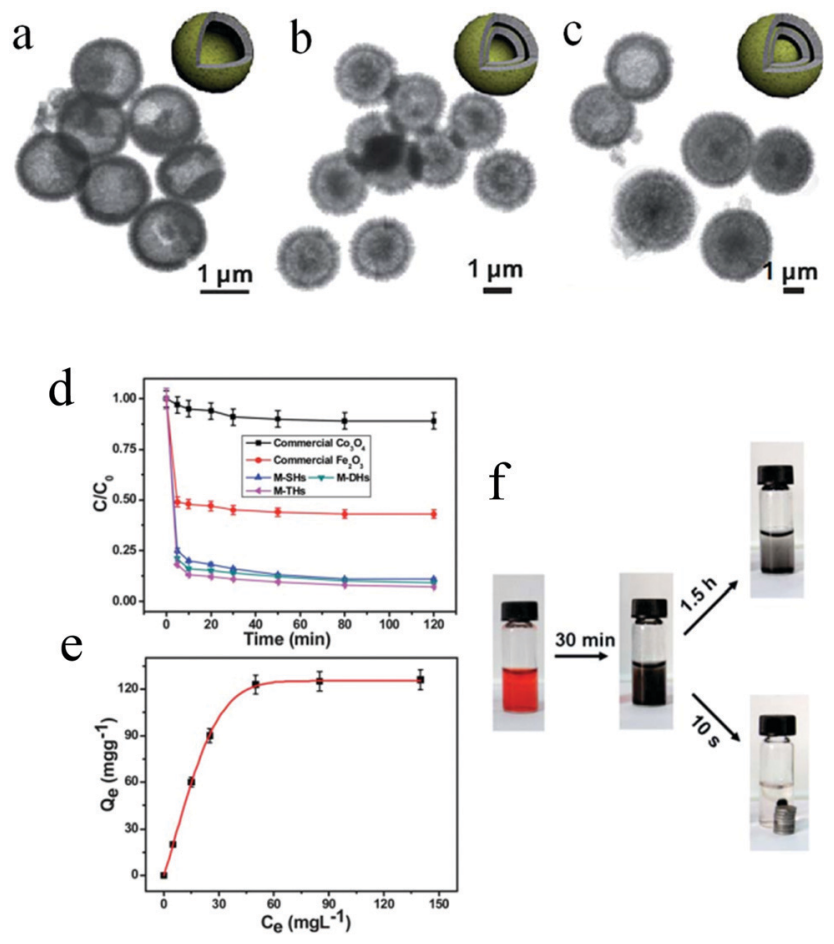

Fig. 47 TEM images of M-MHs: (a) M-SHs, (b) M-DHs, and (c) M-THs. (d) Adsorption rates of Congo red on the three $\mathrm{M}-\mathrm{MHs}$, commercial $\gamma-\mathrm{Fe}_{2} \mathrm{O}_{3}$, and commercial $\mathrm{CO}_{3} \mathrm{O}_{4}$. (e) Adsorption isothermal curves of Congo red on $\mathrm{M}$-THs. (f) Photographs confirming the absorption of Congo red by $\mathrm{M}$-THs with time. Reproduced from ref. 240 with kind permission.

single-shelled (M-SHs), double-shelled (M-DHs), and tripleshelled (M-THs) ensembles. It was found that such M-MHs samples exhibited better adsorptive performance toward the removal of Congo red from water. As shown in Fig. 47d, the adsorption of Congo red on these M-MHs was much higher than those observed using commercial $\gamma-\mathrm{Fe}_{2} \mathrm{O}_{3}$ and commercial $\mathrm{Co}_{3} \mathrm{O}_{4}$. The removal percentages of Congo red were over $75 \%$ and nearly over $90 \%$ in the initial 5 min and within $120 \mathrm{~min}$, respectively, for these three M-MHs samples. However, the 
maximum removal capacities of commercial $\gamma-\mathrm{Fe}_{2} \mathrm{O}_{3}$ and commercial $\mathrm{Co}_{3} \mathrm{O}_{4}$ were about $50 \%$ and $10 \%$, respectively. Meanwhile, the removal capacities were also much higher for these M-MHs. Such superior adsorptive performances of M-MHs might be related to the triple-shelled hollow structures that afforded higher surface areas and stronger electrostatic affinities between their surfaces and Congo red molecules. Fig. 47e shows the isothermal curve of the adsorption of Congo red on M-THs, which conforms to the Langmuir adsorption model. On the basis of this isothermal curve, the maximum adsorption capacity for Congo red was calculated to be $125 \mathrm{mg} \mathrm{g}^{-1}$, which was 3.5 and 20 times those of commercial $\gamma-\mathrm{Fe}_{2} \mathrm{O}_{3}$ and commercial $\mathrm{Co}_{3} \mathrm{O}_{4}$. These improved adsorption performances could be attributed to the multishelled hollow structures and size effect. Last, but not the least, the existence of $\mathrm{Fe}_{3} \mathrm{O}_{4}$ in the M-MHs samples ensured that these M-MHs adsorbents could be conveniently isolated from the solution by using an external magnet in a very short time of about $10 \mathrm{~s}$. If the traditional centrifugation separation procedure was applied, even after $1.5 \mathrm{~h}$, the solution was still not transparent, as shown in Fig. 47f. In addition, the as-prepared M-MHs could also be used for the adsorptive removal of heavily metal ions.

Nowadays, nuclear energy has become increasingly popular in many countries, which is unavoidably accompanied by excessive wastewater containing radionuclides. Because of their mobility and high toxicity, radionuclide contaminants can harm human beings as well as the ecosystem. Therefore, it has become imperative to develop efficient techniques to remove radionuclide pollutants. ${ }^{241,242}$ Adsorption has proven to be one of the most promising methods in the treatment of radionuclidescontaminated water owing to its convenient operation and effectiveness. ${ }^{243,244}$ Recently, Song et al. reported multishelled $\mathrm{Fe}_{3} \mathrm{O}_{4} @ \mathrm{MnO}_{x}$ hollow spheres, which were synthesized via a three-stepped process, namely, the in situ coating of $\mathrm{Fe}_{3} \mathrm{O}_{4} @ \mathrm{C}$ on the surface $\mathrm{SiO}_{2}$ cores, self-template formation of $\mathrm{MnO}_{x}$ by reacting between $\mathrm{Fe}_{3} \mathrm{O}_{4} @ \mathrm{C}$ and $\mathrm{KMnO}_{4}$, and $\mathrm{SiO}_{2}$ removal via etching with $\mathrm{NaOH}^{245}$ When used as an adsorbent for the removal of uranium (U(VI)) and europium (Eu(III)), the isotherms of these two radionuclides onto the adsorbents effectively matched with the Langmuir model. The as-obtained multishelled $\mathrm{Fe}_{3} \mathrm{O}_{4} @ \mathrm{MnO}_{x}$ hollow spheres offered high adsorption capacities (maximum absorption capacities of $106.72 \mathrm{mg} \mathrm{g}^{-1}$ for $\mathrm{U}(\mathrm{VI})$ and $138.13 \mathrm{mg} \mathrm{g}^{-1}$ for $\mathrm{Eu}(\mathrm{III})$ could be obtained at $298 \mathrm{~K}$ ), revealing the advantages of high-performance adsorbents. The adsorption thermodynamics are endothermic processes, while the adsorption kinetics conform to the pseudo-second-order model. It is well known that radionuclides can often coexist with humic acid. The investigation results demonstrate exciting information that the presence of humic acid may provide higher simulation yields of the maximum adsorption capacities toward $\mathrm{U}(\mathrm{VI})$ and $\mathrm{Eu}(\mathrm{III})$, namely, 169.17 and $146.81 \mathrm{mg} \mathrm{g}^{-1}$, respectively. Significantly, the $\mathrm{Fe}_{3} \mathrm{O}_{4} @ \mathrm{MnO}_{2}$ adsorbent could be easily recovered by using an external magnet for successive usage.

Apart from heavy metal ions and organic pollutants, multishelled hollow nanostructures can also be used for the removal of gaseous pollutants. Utilizing carbonaceous microspheres as the hard template, Feng et al. fabricated nanostructures with controlled $\mathrm{Ca} / \mathrm{Mg}$ molar ratio, number of shells, and size. ${ }^{246}$ The Mg-modified $\mathrm{CaCO}_{3}$ multishelled hollow microspheres exhibited remarkable $\mathrm{CO}_{2}$ adsorption performance. Under optimized conditions, triple-shelled Mg-modified $\mathrm{CaCO}_{3}$ provides adsorption capacity of $0.51 \mathrm{~g} \mathrm{CO}_{2} \mathrm{~g}^{-1}$ adsorbent, which is marginally less than that of commercial $\mathrm{CaCO}_{3}-\mathrm{C}\left(0.53 \mathrm{~g} \mathrm{CO}_{2} \mathrm{~g}^{-1}\right.$ adsorbent). Nevertheless, owing to the conversion of $\mathrm{CaCO}_{3}$ into $\mathrm{CaO}$ at higher temperatures, the serious sintering of commercial $\mathrm{CaCO}_{3}-\mathrm{C}$ results in a rapid decay in the adsorption capacity for $\mathrm{CO}_{2}$ from the second cycle. Because of the introduction of $\mathrm{Mg}$, the sintering and aggregation of $\mathrm{CaO}$ nanoparticles at high temperature could be efficiently prevented due to the high Tammann temperature of $\mathrm{MgO}$. As a result, these multishelled Mg-modified $\mathrm{CaCO}_{3}$ microspheres could maintain the $\mathrm{CO}_{2}$ adsorption capacity after repeated cycles.

\subsection{Chemical catalysis for organic transformation}

Via a layer-by-layer deposition process followed by alkali etching, Liu and co-workers prepared double-shelled $\mathrm{CeO}_{2} / \mathrm{M} @ \mathrm{M} / \mathrm{TiO}_{2}$ $(\mathrm{M}=\mathrm{Au}$ and/or $\mathrm{Pd})$ nanospheres, which exhibited excellent catalytic performance toward several organic reactions, such as Suzuki reaction, aerobic oxidation of benzyl alcohol, and reduction of 4-nitrophene. ${ }^{247} \mathrm{Xu}$ et al. prepared CSs containing $\mathrm{Ce}^{3+}$ by a two-stepped hydrothermal method using glucose as the carbon precursor and $\mathrm{Ce}\left(\mathrm{NO}_{3}\right)_{3}$ as the $\mathrm{Ce}$ precursor and then converted them into multishelled $\mathrm{CeO}_{2}$ hollow spheres via controlled calcination in an air/gas flow. Au nanoparticles were deposited and dispersed on these multishelled $\mathrm{CeO}_{2}$ hollow spheres at a high content. The $\mathrm{Au} / \mathrm{CeO}_{2}$ hollow spheres exhibited higher catalytic activity and more stability toward the reduction of 4-nitrophenol with $\mathrm{NaBH}_{4}$ than those from other noble-metal-loaded catalysts in other structures. Significantly, the $\mathrm{Au} / \mathrm{CeO}_{2}$ catalyst showed no changes in both morphology and activity after eight cycles. This can be attributed to the hierarchically nanoscale sizes of the multishelled $\mathrm{CeO}_{2}$ hollow sphere support and the highly dispersed ultrathin $\mathrm{Au}$ nanoparticles. ${ }^{248}$ Liao et al. synthesized Ce-based coordination polymer by a mixed-solvothermal process and then converted it into multishelled $\mathrm{CeO}_{2}$ hollow nanospheres via a simple thermal treatment in air. The prepared multishelled $\mathrm{CeO}_{2}$ hollow nanospheres not only exhibited efficient photocatalytic activity for the degradation of rhodamine $\mathrm{B}$ under visible light but also could be used as a support to load noble metal nanoparticles (such as Au and AuPd). When used as a catalyst, $\mathrm{Au} / \mathrm{CeO}_{2}$ showed high activity toward the reduction of 4-nitrophenol at a reaction rate constant of $0.416 \mathrm{~min}^{-1}$, and $\mathrm{AuPd} / \mathrm{CeO}_{2}$ are efficient catalysts for $\mathrm{CO}$ oxidation. ${ }^{249}$ Zhang et al. introduced $\mathrm{Pt}$ species into double-shelled $\mathrm{TiO}_{2}$ hollow spheres, which was prepared via a simple hydrothermal treatment of $\mathrm{SiO}_{2} @ \mathrm{TiO}_{2}\left(\mathrm{TiO}_{2}\right.$-coated solid $\mathrm{SiO}_{2}$ spheres $){ }^{250}$ The as-prepared Pt (DHS-TiPt) composite showed excellent catalytic activity toward the reduction of 4-NP with $\mathrm{NaBH}_{4}$.

Similar to gas sensor applications, by precisely controlling the pore size distribution, multishelled hollow nanostructures could selectively permit reactant/product diffusion through the 
shells, resulting in outstanding selective catalysis processes. In aqueous solutions, the hydrophilic characteristics of the shell influence the reactants with specific groups accessing the interior of such multishelled hollow nanostructures, further improving selectivity. In addition, multiple thin shells with large intershell spacings can provide a large surface area, resulting in more reactants contacting the active site and therefore enhancing the catalytic performance.

\subsection{Drug delivery}

Drug delivery, aimed at improving the aqueous solubility, chemical stability, and pharmacological activity, as well as reducing the side-effects of drugs, can be used to transport therapeutic drugs in the body as needed to safely achieve the desired therapeutic effect. The goal of any drug delivery system is to provide and maintain the therapeutic concentrations of drug at the target biological site. ${ }^{251,252}$

Multishelled hollow nanostructures with tunable pore and shell structures have been widely employed for drug delivery. For drug delivery systems, three important parameters are the most significant: loading capacity, good targeting, and sustained drug release. These evaluation parameters can be influenced by the number, thickness, and porosity of shells. Multiple thin shells can provide abundant loading sites, leading to high drug loading capacity. Porous shells allow multishelled hollow nanocarriers to reach the target drug molecules with preferred sizes. In addition, multishelled hollow nanostructures with adjustable hydrophilic and hydrophobic properties can allow the co-loading/release of various drugs.

For example, Wu et al. synthesized multishelled mesoporous silica spheres by the vesicle template method. When used as the carriers of anticancer drugs, the loading efficiency of doxorubicin hydrochloride could reach up to $83 \%$ at the optimum loading concentration of $1.2 \mathrm{mg} \mathrm{L}{ }^{-1}$. More significantly, the loaded anticancer drug exhibited the desired pH-responsive release behavior. In particular, the release rate under acidic conditions is much higher than that under basic conditions, which is beneficial for killing cancer cells since almost all the cancer cells have an acidic intracellular environment. Huang et al. demonstrated that hollow triple-shelled mesoporous silica spheres with tunable shell-to-shell distances loaded with doxorubicin hydrochloride yielded excellent performance in killing cancer cells by a slow, controlled, and sustained release. ${ }^{253}$

\section{Conclusion and outlook}

In summary, multishelled hollow nanostructures have become a hot topic in both the scientific arena and engineering applications. Substantial progress has been made in the past few decades with the development of efficient methods to construct multishelled hollow nanostructures. In this review, we have provided a comprehensive overview of the synthesis strategies and applications of multishelled hollow nanostructures. For further research in the field of multishelled hollow nanostructures, the focus should be on the following aspects.
As far as synthesis is concerned, it might always be challenging to precisely control and manipulate multishelled hollow nanostructures by means of simple and cost-effective approaches such that they can have the desired compositions and morphologies. Several solutions can be considered. For one thing, further expanding and modifying the existing template-based/ template-free approaches are expected to be developed. For another thing, the combination of various approaches containing multiple hollowing mechanisms in a single synthesis process can undoubtedly be the trend for fabricating certain multishelled hollow nanomaterials with special structures and properties. Furthermore, despite several challenges, novel strategies for generating multishelled hollow structures can be developed, bringing revolutionary advances to this area. For the applications of multishelled hollow nanostructures, engineers and scientists can investigate the relationship among the structures of such complex hollow materials as well as enhanced performances. Meanwhile, for practical applications of such promising materials, the situation for multishelled hollow nanostructures might be fairly different, which should be effectively considered, too. In particular, energy-related applications often consider ion/electron and charge/mass transports as well as the capability of immobilizing sulfide/ lithium or electrolyte ions by chemical/physical means. For catalyst/adsorbent materials, the activity and reusability should be seriously considered. Carrier materials for certain drugs heavily rely on biocompatibility as well as interaction between the drug molecules. Consequently, a comprehensive understanding of the requirements of specific applications is expected to, in turn, direct the fabrication of multishelled hollow nanomaterials. It is believed that multishelled hollow nanostructures, as a type of versatile functional materials, can have a bright future in many fields.

\section{Conflicts of interest}

The authors declare no competing financial interest.

\section{Acknowledgements}

This work was financially supported by the National Natural Science Foundation of China (Grant No. 21403091, and 51473070).

\section{References}

1 X.-J. Wang, J. Feng, Y.-C. Bai, Q. Zhang and Y.-D. Yin, Synthesis, Properties, and Applications of Hollow Micro-/ Nanostructures, Chem. Rev., 2016, 116, 10983-11060.

2 Q. Zhang, W.-S. Wang, J. Goebl and Y.-D. Yin, Selftemplated Synthesis of Hollow Nanostructures, Nano Today, 2009, 4, 494-507.

3 T. Liu, L. Zhang, B. Cheng and J. Yu, Hollow Carbon Spheres and Their Hybrid Nanomaterials in Electrochemical Energy Storage, Adv. Energy Mater., 2019, 9, 1803900. 
4 L. Zhou, Z.-C. Zhuang, H.-H. Zhao, M.-T. Lin, D.-Y. Zhao and L.-Q. Mai, Intricate Hollow Structures: Controlled Synthesis and Applications in Energy Storage and Conversion, Adv. Mater., 2017, 29, 1602914.

5 X.-Y. Yu, L. Yu and X.-W. Lou, Metal Sulfide Hollow Nanostructures for Electrochemical Energy Storage, $A d v$. Energy Mater., 2016, 6, 1501333.

6 L. Yu., X.-Y. Yu and X.-W. Lou, The Design and Synthesis of Hollow Micro-/Nanostructures: Present and Future Trend, Adv. Mater., 2018, 30, 1800939.

7 L. Yu, H. Hu, H.-B. Wu and X.-W. Lou, Complex Hollow Nanostructures: Synthesis and Energy-Related Applications, Adv. Mater., 2017, 29, 1604563.

8 X.-Y. Yu, L. Yu and X.-W. Lou, Hollow Nanostructures of Molybdenum Sulfides for Electrochemical Energy Storage and Conversion, Small Methods, 2017, 1, 1600020.

9 D. Mao, J.-W. Wan, J.-Y. Wang and D. Wang, Sequential Templating Approach: A Groundbreaking Strategy to Create Hollow Multishelled Structures, Adv. Mater., 2018, 1802874.

10 H.-X. Yang, J.-F. Qian, Z.-X. Chen, X.-P. Ai and Y.-L. Cao, Multilayered Nanocrystalline $\mathrm{SnO}_{2}$ Hollow Microspheres Synthesized by Chemically Induced Self-Assembly in the Hydrothermal Environment, J. Phys. Chem. C, 2007, 111, 14067-14071.

11 X.-Y. Lai, J. E. Halpert and D. Wang, Recent Advances in Micro-/Nano-tructured Hollow Spheres for Energy Applications: From Simple to Complex Systems, Energy Environ. Sci., 2012, 5, 5604-5618.

12 A.-B. Chen, W.-P. Zhang, Y. Liu, X.-W. Han and X.-H. Bao, In Situ Introduction of Dispersed Metallic Ag Nanoparticles into the Channels of Mesoporous Carbon CMK-3, Chin. Chem. Lett., 2007, 18, 1017-1020.

13 J.-S. Wu and D.-F. Xue, Hierarchical Integration of $\mathrm{ZnO}$ Nanocrystals into Multishelled Superstructures, Nanosci. Nanotechnol. Lett., 2011, 3, 371-377.

14 Z.-M. Li, X.-Y. Lai, H. Wang, D. Mao, C.-J. Xing and D. Wang, General Synthesis of Homogeneous Hollow Core-Shell Ferrite Microspheres, J. Phys. Chem. C, 2009, 113, 2792-2797.

15 H.-L. Xu and W.-Z. Wang, Template Synthesis of Multishelled $\mathrm{Cu}_{2} \mathrm{O}$ Hollow Spheres with a Single-Crystalline Shell Wall, Angew. Chem., Int. Ed., 2007, 46, 1489-1492.

16 J. Qi, X.-Y. Lai, J.-Y. Wang, H.-J. Tang, H. Ren, Y. Yang, Q. Jin, L.-J. Zhang, R.-B. Yu, G.-H. Ma, Z.-G. Su, H.-J. Zhao and D. Wang, Multi-shelled Hollow Micro-/Nanostructures, Chem. Soc. Rev., 2015, 44, 6749-6773.

17 M. Qin, D. Lan, J.-L. Liu, H.-S. Liang, L.-M. Zhang, H. Xing, T.-T. Xu and H.-J. Wu, Synthesis of Single-component Metal Oxides with Controllable Multi-shelled Structure and their Morphology-related Applications, Chem. Rec., 2019, 19, 1-19.

18 H. Ren and R.-B. Yu, Hollow Multi-shelled Structures for Energy Conversion and Storage Applications, Inorg. Chem. Front., 2019, 6, 2239-2259.

19 Y. Liu, X.-C. Li, W.-M. Shen, Y. Dai, W. Kou, W.-J. Zheng, X.-B. Jiang and G.-H. He, Multishelled Transition Metal-based
Microspheres: Synthesis and Applications for Batteries and Supercapacitors, Small, 2019, 15, 1804737.

20 J.-Y. Wang, H.-J. Tang, H. Wang, R.-B. Yu and D. Wang, Multi-shelled Hollow Micro-/anostructures: Promising Platforms for Lithium-ion Batteries, Mater. Chem. Front., 2017, 1, 414-430.

21 J.-Y. Wang, J.-W. Wan and D. Wang, Hollow Multishelled Structures for Promising Applications: Understanding the Structure-Performance Correlation, Acc. Chem. Res., 2019, 52, 2169-2178.

22 S. H. Hwang, J. Yun and J. Jang, Multi-Shell Porous $\mathrm{TiO}_{2}$ Hollow Nanoparticles for Enhanced Light Harvesting in Dye-sensitized Solar Cells, Adv. Funct. Mater., 2014, 24, 7619-7626.

23 X.-W. Lou, C.-L. Yuan and L. A. Archer, Shell-by-Shell Synthesis of Tin Oxide Hollow Colloids with Nanoarchitectured Walls: Cavity Size Tuning and Functionalization, Small, 2007, 3, 261-265.

24 X.-Y. Yu, L. Yu, L.-F. Shen, X.-H. Song, H.-Y. Chen and X.-W. Lou, General Formation of MS (M = Ni, Cu, Mn) Boxin-Box Hollow Structures with Enhanced Pseudocapacitive Properties, Adv. Funct. Mater., 2014, 24, 7440-7446.

25 N.-X. Du, W.-J. Zheng, X.-C. Li, G.-H. He, L. Wang and J.-H. Shi, Nanosheet-assembled NiS Hollow Structures with Double Shells and Controlled Shapes for High-performance Supercapacitors, Chem. Eng. J., 2017, 323, 415-424.

26 X. Li, Y. Zhang, H.-J. Zhang, Y.-Y. Feng and Y. Wang, Porous Double-shelled $\mathrm{SnO}_{2}$ @C Hollow Spheres as HighPerformance Anode Material for Lithium Ion Batteries, Electrochim. Acta, 2016, 195, 208-215.

27 Z. Sun, X.-F. Song, P. Zhang and L. Gao, Template-assisted Synthesis of Multi-shelled Carbon Hollow Spheres with an Ultralarge Pore Volume as Anode Materials in Li-ion Batteries, RSC Adv., 2015, 5, 3657-3664.

28 X.-Y. Lai, J. Li, B. A. Korgel, Z.-H. Dong, Z.-M. Li, F.-B. Su, J. Du and D. Wang, General Synthesis and Gas-Sensing Properties of Multiple-Shell Metal Oxide Hollow Microspheres, Angew. Chem., Int. Ed., 2011, 50, 2738-2741.

29 H.-M. Sun, L.-M. Wang, D.-Q. Chu, Z.-C. Ma and A.-X. Wang, Facile Fabrication of Multishelled $\mathrm{Cr}_{2} \mathrm{O}_{3}$ Hollow Microspheres with Enhanced Gas Sensitivity, Mater. Lett., 2015, 140, 158-161.

30 T. Gan, A.-X. Zhao, S.-H. Wang, Z. Lv and J.-Y. Sun, Hierarchical Triple-shelled Porous Hollow Zinc Oxide Spheres Wrapped in Graphene Oxide as Efficient Sensor Material for Simultaneous Electrochemical Determination of Synthetic Antioxidants in Vegetable Oil, Sens. Actuators, B, 2016, 235, 707-716.

31 H.-J. Wu, G.-L. Wu, Q.-F. Wu and L.-D. Wang, Facile Synthesis and Microwave Absorbability of C@Ni-NiO Core-shell Hybrid Solid Sphere and Multi-shelled NiO Hollow Sphere, Mater. Charact., 2014, 97, 18-26.

32 H.-J. Wu, Y.-Q. Wang, C.-H. Zheng, J.-M. Zhu, G.-L. Wu and X.-H. Li, Multi-shelled NiO Hollow Spheres: Easy Hydrothermal Synthesis and Lithium Storage Performances, J. Alloys Compd., 2016, 685, 8-14. 
33 Z.-D. Wang, S.-H. Qu, Y.-H. Cheng, C.-H. Zheng, S.-Y. Chen and H.-J. Wu, Facile Synthesis of $\mathrm{Co}_{3} \mathrm{O}_{4}$ Spheres and their Unexpected High Specific Discharge Capacity for Lithiumion Batteries, Appl. Surf. Sci., 2017, 416, 338-343.

34 Z.-H. Dong, X.-Y. Lai, J. E. Halpert, N.-L. Yang, L.-X. Yi, J. Zhai, D. Wang, Z.-Y. Tang and L. Jiang, Accurate Control of Multishelled ZnO Hollow Microspheres for Dye-Sensitized Solar Cells with High Efficiency, Adv. Mater., 2012, 24, 1046-1049.

35 H. Ren, R.-B. Yu, J.-Y. Wang, Q. Jin, M. Yang, D. Mao, D. Kisailus, H.-J. Zhao and D. Wang, Multishelled $\mathrm{TiO}_{2}$ Hollow Microspheres as Anodes with Superior Reversible Capacity for Lithium Ion Batteries, Nano Lett., 2014, 14, 6679-6684.

36 S.-M. Xu, C. M. Hessel, H. Ren, R.-B. Yu, Q. Jin, M. Yang, H.-J. Zhao and D. Wang, $\alpha-\mathrm{Fe}_{2} \mathrm{O}_{3}$ Multi-shelled Hollow Microspheres for Lithium ion Battery Anodes with Superior Capacity and Charge Retention, Energy Environ. Sci., 2014, 7, 632-637.

37 S.-S. Niu, Y. Wang, S. Lu, D.-X. Wang and P. Wang, Fabrication and Photocatalytic Properties of $\mathrm{SnO}_{2}$ Double-shelled and Triple-shelled Hollow Spheres, Solid State Sci., 2016, 56, 63-67.

38 Z.-H. Dong, H. Ren, C. M. Hessel, J.-Y. Wang, R.-B. Yu, Q. Jin, M. Yang, Z.-D. Hu, Y.-F. Chen, Z.-Y. Tang, H.-J. Zhao and D. Wang, Quintuple-Shelled $\mathrm{SnO}_{2}$ Hollow Microspheres with Superior Light Scattering for High-Performance Dye-Sensitized Solar Cells, Adv. Mater., 2014, 26, 905-909.

39 J.-Y. Wang, Y. Cui and D. Wang, Design of Hollow Nanostructures for Energy Storage, Conversion and Production, Adv. Mater., 2019, 31, 1801993.

40 J. Wang, N. Yang, H. Tang, Z. Dong, Q. Jin, M. Yang, D. Kisailus, H. Zhao, Z. Tang and D. Wang, Accurate Control of Multishelled $\mathrm{Co}_{3} \mathrm{O}_{4}$ Hollow Microspheres as High Performance Anode Materials in Lithium-Ion Batteries, Angew. Chem., Int. Ed., 2013, 52, 6417-6420.

41 X.-X. Zhao, R.-B. Yu, H.-J. Tang, D. Mao, J. Qi, B. Wang, Y. Zhang, H.-J. Zhao, W.-P. Hu and D. Wang, Formation of Septuple-Shelled $\left(\mathrm{Co}_{2 / 3} \mathrm{Mn}_{1 / 3}\right)\left(\mathrm{Co}_{5 / 6} \mathrm{Mn}_{1 / 6}\right)_{2} \mathrm{O}_{4}$ Hollow Spheres as Electrode Material for Alkaline Rechargeable Battery, Adv. Mater., 2017, 29, 1700550.

42 Y.-P. Wang, A.-Q. Pan, Q.-Y. Zhu, Z.-W. Nie, Y.-F. Zhang, Y. Tang, S.-Q. Liang and G.-Z. Cao, Facile Synthesis of Nanorod-assembled Multi-shelled $\mathrm{Co}_{3} \mathrm{O}_{4}$ Hollow Microspheres for High-performance Supercapacitors, J. Power Sources, 2014, 272, 107-112.

43 J.-Y. Wang, H.-J. Tang, H. Ren, R.-B. Yu, J. Qi, D. Mao, H.J. Zhao and D. Wang, pH-Regulated Synthesis of MultiShelled Manganese Oxide Hollow Microspheres as Supercapacitor Electrodes Using Carbonaceous Microspheres as Templates, Adv. Sci., 2014, 1, 1400011.

44 Z.-P. Tian, Y. Zhou, Z.-D. Li, Q. Liu and Z.-G. Zou, Generalized Synthesis of a Family of Multishelled Metal Oxide Hollow Microspheres, J. Mater. Chem. A, 2013, 1, 3575-3579.

45 L.-H. Chu, M.-C. Li, Z.-P. Wan, L. Ding, D.-D. Song, S.-Y. Dou, J.-W. Chen and Y. Wang, Morphology Control and
Fabrication of Multi-shelled NiO Spheres by Tuning the $\mathrm{pH}$ Value via a Hydrothermal Process, CrystEngComm, 2014, 16, 11096-11101.

46 G.-Q. Zhang and X.-W. Lou, General Synthesis of MultiShelled Mixed Metal Oxide Hollow Spheres with Superior Lithium Storage Properties, Angew. Chem., Int. Ed., 2014, 53, 9041-9044.

47 L.-X. Zhang, Y.-X. Sun, W.-B. Jia, S.-S. Ma, B. Song, Y. Li, H.-F. Jiu and J.-W. Liu, Multiple Shell Hollow $\mathrm{CoFe}_{2} \mathrm{O}_{4}$ Spheres: Synthesis, Formation Mechanism and Properties, Ceram. Int., 2014, 40, 8997-9002.

48 S.-J. Peng, F. Gong, L.-L. Li, D.-S. Yu, D.-X. Ji, T.-R. Zhang, Z. Hu, Z.-Q. Zhang, S.-L. Chou, Y.-H. Du and S. Ramakrishna, Necklace-like Multishelled Hollow Spinel Oxides with Oxygen Vacancies for Efficient Water Electrolysis, J. Am. Chem. Soc., 2018, 140, 13644-13653.

49 J.-Y. Wang, H.-J. Tang, L.-J. Zhang, H. Ren, R.-B. Yu, Q. Jin, J. Qi, D. Mao, M. Yang, Y. Wang, P.-R. Liu, Y. Zhang, Y.-R. Wen, L. Gu, G.-H. Ma, Z.-G. Su, Z.-Y. Tang, H.-J. Zhao and D. Wang, Multi-shelled Metal Oxides Prepared via an Anion-adsorption Mechanism for Lithium-ion Batteries, Nat. Energy, 2016, 1, 16050.

50 X.-X. Zhao, J.-Y. Wang, R.-B. Yu and D. Wang, Construction of Multishelled Binary Metal Oxides via Coabsorption of Positive and Negative Ions as a Superior Cathode for Sodium-Ion Batteries, J. Am. Chem. Soc., 2018, 140, 17114-17119.

51 L.-B. Zong, J. Xu, S.-Y. Jiang, K. Zhao, Z.-M. Wang, P.-R. Liu, H.-J. Zhao, J. Chen, X.-R. Xing and R.-B. Yu, Composite Yttrium-carbonaceous Spheres Templated Multi-Shell $\mathrm{YVO}_{4}$ Hollow Spheres with Superior Upconversion Photoluminescence, Adv. Mater., 2017, 29, 1604377.

52 J. Zhang, Y.-D. Cao, C.-A. Wang and R. Ran, Design and Preparation of $\mathrm{MnO}_{2} / \mathrm{CeO}_{2}-\mathrm{MnO}_{2}$ Double-Shelled Binary Oxide Hollow Spheres and their Application in CO Oxidation, ACS Appl. Mater. Interfaces, 2016, 8, 8670-8677.

53 M. Yang, J. Ma, C.-L. Zhang, Z.-Z. Yang and Y.-F. Lu, General Synthetic Route toward Functional Hollow Spheres with Double-Shelled Structures, Angew. Chem., Int. Ed., 2005, 44, 6727-6730.

54 H.-B. Hou, L.-P. Zhang, T.-M. Liu, J.-M. Cheng, L.-Q. Sun, C.-D. Wang, M.-M. Jin, Z.-X. Song, J.-Y. Cheng, Q.-G. Wang, H.-G. Sun, X. Chen and G.-L. Cui, A Facile Approach to Preparation of Silica Double-Shell Hollow Particles, and their Application in Gel Composite Electrolytes, J. Colloid Interface Sci., 2018, 529, 130-138.

55 M. Yang, J. Ma, Z. Niu, X. Dong, H. Xu, Z. Meng, Z. Jin, Y. Lu, Z. Hu and Z. Yang, Synthesis of Spheres with Complex Structures Using Hollow Latex Cages as Templates, Adv. Funct. Mater., 2005, 15, 1523-1528.

56 M. Yang, J. Ma, C.-L. Zhang, Z.-Z. Yang and Y.-F. Lu, General Synthetic Route toward Functional Hollow Spheres with Double-Shelled Structures, Angew. Chem., Int. Ed., 2005, 44, 6727-6730.

57 M.-Y. Bai, Y.-J. Cheng, S. A. Wickline and Y.-N. Xia, Colloidal Hollow Spheres of Conducting Polymers with Smooth 
Surface and Uniform, Controllable Sizes, Small, 2009, 5, 1747-1752.

58 M.-Y. Bai and Y.-N. Xia, Facile Synthesis of Double-Shelled Polypyrrole Hollow Particles with a Structure Similar to that of a Thermal Bottle, Macromol. Rapid Commun., 2010, 31, 1863-1868.

59 Y. Zeng, X. Wang, H. Wang, Y. Dong, Y. Ma and J.-N. Yao, Multi-shelled Titania Hollow Spheres Fabricated by a Hard Template Strategy: Enhanced Photocatalytic Activity, Chem. Commun., 2010, 46, 4312-4314.

60 X.-C. Li, L. Wang, J.-H. Shi, N.-X. Du and G.-H. He, Multishelled Nickel-Cobalt Oxide Hollow Microspheres with Optimized Compositions and Shell Porosity for HighPerformance Pseudocapacitors, ACS Appl. Mater. Interfaces, 2016, 8, 17276-17283.

61 X.-H. Qi, W.-J. Zheng, G.-H. He, T.-F. Tian, N.-X. Du and L. Wang, $\mathrm{NiCo}_{2} \mathrm{O}_{4}$ Hollow Microspheres with Tunable Numbers and Thickness of Shell for Supercapacitors, Chem. Eng. J., 2017, 309, 426-434.

62 K.-X. Zhu, C.-Z. Jin, C.-X. Zhao, R.-S. Hu, Z. Klencsár, G. A. Sundaram, D. F. Srankóf, R. Ge and J.-H. Wang, Modulation Synthesis of Multi-shelled Cobalt-iron Oxides as Efficient Catalysts for Peroxymonosulfate-mediated Organics Degradation, Chem. Eng. J., 2019, 359, 1537-1549.

63 Y. Qi, B.-Q. Liu, L.-Y. Zhang, Y.-Q. Huo, L. Li, H.-M. Xie, C.-G. Wang and Z.-M. Su, One-pot Controllable Synthesis of $\mathrm{CoFe}_{2} \mathrm{O}_{4}$ Solid, Hollow and Multi-shell Hollow Nanospheres as Superior Anode Materials for Lithium ion Batteries, J. Mater. Chem. A, 2017, 5, 21994-22003.

64 J. Sun, C.-X. Lv, F. Lv, S. Chen, D.-H. Li, Z.-Q. Guo, W. Han, D.-J. Yang and S.-J. Guo, Tuning the Shell Number of Multishelled Metal Oxide Hollow Fibers for Optimized Lithium-Ion Storage, ACS Nano, 2017, 11, 6186-6193.

65 L.-F. Wang, F.-J. Liu, W.-T. Yang, H.-Y. Zhao, Y.-Y. Zheng, X.-B. Chen and W.-J. Dong, Synthesis of Multiple-shell Porous $\mathrm{CeO}_{2}$ Hollow Spheres by a Hydrogel Template Method, Mater. Lett., 2013, 107, 42-45.

66 F.-Z. Mou, J.-G. Guan, W.-D. Shi, Z.-G. Sun and S.-H. Wang, Oriented Contraction: A Facile Nonequilibrium HeatTreatment Approach for Fabrication of Maghemite Fiberin-Tube and Tube-in-Tube Nanostructures, Langmuir, 2010, 26, 15580-15585.

67 R. Pang, X.-J. Hu, S.-Y. Zhou, C.-H. Sun, J. Yan, X.-M. Sun, S.-Z. Xiao and P. Chen, Preparation of Multi-shelled Conductive Polymer Hollow Microspheres by Using $\mathrm{Fe}_{3} \mathrm{O}_{4}$ Hollow Spheres as Sacrificial Templates, Chem. Commun., 2014, 50, 12493-12496.

68 C.-Y. Niu, B.-F. Zou, Y.-Q. Wang, L. Chen, H.-H. Zheng and S.-M. Zhou, The Template-assisted Aynthesis of Polypyrrole Hollow Microspheres with a Double-shelled Structure, Chem. Commun., 2015, 51, 5009-5012.

69 K.-L. Zhang, X.-N. Li, J.-W. Liang, Y.-C. Zhu, L. Hu, Q.-S. Cheng, C. Guo, N. Lin and Y.-T. Qian, Nitrogen-doped Porous Interconnected Double-shelled Hollow Carbon Spheres with High Capacity for Lithium Ion Batteries and Sodium Ion Batteries, Electrochim. Acta, 2015, 155, 174-182.
70 G.-M. Zhou, Y.-B. Zhao and A. Manthiram, Dual-Confined Flexible Sulfur Cathodes Encapsulated in Nitrogen-Doped Doubl-Shelled Hollow Carbon Spheres and Wrapped with Graphene for Li-S Batteries, Adv. Energy Mater., 2015, 5, 1402263.

71 R. G. Chaudhuri and S. Paria, Au and Ag/Au Double-shells Hollow Nanoparticles with Improved near Infrared Surface Plasmon and Photoluminescence Properties, J. Colloid Interface Sci., 2016, 461, 15-19.

72 Y. J. Wong, L.-F. Zhu, W. S. Teo, Y. W. Tan, Y.-H. Yang, C. Wang and H.-Y. Chen, Revisiting the Stöber Method: Inhomogeneity in Silica Shells, J. Am. Chem. Soc., 2011, 133, 11422-11425.

73 D. Gu, H. Bongard, Y.-H. Deng, D. Feng, Z.-X. Wu, Y. Fang, J.-J. Mao, B. Tu, F. Schuth and D. Y. Zhao, An Aqueous Emulsion Route to Synthesize Mesoporous Carbon Vesicles and Their Nanocomposites, Adv. Mater., 2010, 22, 833-837.

74 J. Liu, T.-Y. Yang, D.-W. Wang, G.-Q. Lu, D.-Y. Zhao and S.-Z. Qiao, A Facile Soft-template Synthesis of Mesoporous Polymeric and Carbonaceous Nanospheres, Nat. Commun., 2013, 4, 2798.

75 H.-L. Xu and W.-Z. Wang, Template Synthesis of Multishelled $\mathrm{Cu}_{2} \mathrm{O}$ Hollow Spheres with a Single-Crystalline Shell Wall, Angew. Chem., Int. Ed., 2007, 46, 1489-1492.

76 B.-S. Wang, W.-W. Zhang, Z.-Y. Zhang, R.-Y. Li, Y.-L. Wu, Z.-G. Hu, X.-L. Wu, C.-G. Guo, G.-A. Cheng and R.-T. Zheng, $\mathrm{Cu}_{2} \mathrm{O}$ Hollow Structures-microstructural Evolution and Photocatalytic Properties, RSC Adv., 2016, 6, 103700.

77 Z.-G. Teng, X.-D. Su, Y.-Y. Zheng, J.-J. Zhang, Y. Liu, S.-J. Wang, J. Wu, G.-T. Chen, J.-D. Wang, D.-Y. Zhao and G.-M. Lu, A Facile Multi-interface Transformation Approach to Monodisperse Multiple-Shelled Periodic Mesoporous Organosilica Hollow Spheres, J. Am. Chem. Soc., 2015, 137, 7935-7944.

78 J. Yang, X. Wang, R. Xia, E. Muhire and M.-Z. Gao, Selfassembly in the Synthesis of Shelled ZnO Hollow Spheres and Their UV Sensors Performance, Mater. Lett., 2016, 182, 10-14.

79 Y. Zhang, M.-H. Yu, L. Zhou, X.-F. Zhou, Q.-F. Zhao, H.-X. Li and C.-Z. Yu, Organosilica Multilamellar Vesicles with Tunable Number of Layers and Sponge-Like Walls via One Surfactant Templating, Chem. Mater., 2008, 20, 6238-6243.

80 C.-P. Xu, D.-C. Niu, N. Zheng, H.-N. Yu, J.-P. He and Y.-S. Li, Facile Synthesis of Nitrogen-Doped Double-Shelled Hollow Mesoporous Carbon Nanospheres as High-Performance Anode Materials for Lithium Ion Batteries, ACS Sustainable Chem. Eng., 2018, 6, 5999-6007.

81 J. Liu, S. B. Hartono, Y.-G. Jin, Z. Li, G.-Q. Lu and S.-Z. Qiao, A Facile Vesicle Template Route to Multi-shelled Mesoporous Silica Hollow Nanospheres, J. Mater. Chem., 2010, 20, 4595-4601.

82 L. Wu, H.-J. Zhang, M.-H. Wu, Y.-F. Zhong, X.-W. Liu and Z. Jiao, Dual-templating Synthesis of Multi-shelled Mesoporous Silica Nanoparticles as Catalyst and Drug Carrier, Microporous Mesoporous Mater., 2016, 228, 318-328. 
83 G.-W. Zhou, Y.-J. Chen and J.-H. Yang, and S.-H. Yang, From Cylindrical-channel Mesoporous Silica to Vesicle-like Silica with Well-defined Multilamella Shells and Large Inter-shell Mesopores, J. Mater. Chem., 2007, 17, 2839-2844.

84 X. Gu, C.-L. Li, X.-H. Liu, J.-W. Ren, Y.-Q. Wang, Y.-L. Guo, Y. Guo and G.-Z. Lu, Synthesis of Nanosized Multilayered Silica Vesicles with High Hydrothermal Stability, J. Phys. Chem. C, 2009, 113, 6472-6479.

85 Y. Zhang, G.-W. Zhou, B. Sun, M.-N. Zhao, J.-Y. Zhang and F. J. Chen, A Cationic-cationic Co-surfactant Templating Route for Synthesizing Well-defined Multilamellar Vesicular Silica with an Adjustable Number of Layers, Chem. Commun., 2014, 50, 2907-2909.

86 Y.-N. Yang, Y. Lu, P. L. Abbaraju, J. Zhang, M. Zhang, G.-Y. Xiang and C.-Z. Yu, Multi-shelled Dendritic Mesoporous Organosilica Hollow Spheres: Roles of Composition and Architecture in Cancer Immunotherapy, Angew. Chem., Int. Ed., 2017, 56, 8446-8450.

87 S. Yang, X.-F. Zhou, P. Yuan, M.-H. Yu, S.-H. Xie, J. Zou, G.-Q. $\mathrm{Lu}$ and C.-Z. Yu, Siliceous Nanopods from a Compromised Dual-Templating Approach, Angew. Chem., Int. Ed., 2007, 46, 8579-8582.

88 X. Huang, W. Li, M.-J. Wang, X.-N. Tan, Q. Wang, M.-N. Zhang, C. Wang and H.-F. Zhang, Synthesis of Multipleshelled Organosilica Hollow Nanospheres via a Dualtemplate Method by Using Compressed $\mathrm{CO}_{2}$, Microporous Mesoporous Mater., 2017, 247, 66-74.

89 T.-T. Zhou, T. Zhang, R. Zhang, Z. Lou, J.-N. Deng and L.-L. Wang, Hollow $\mathrm{ZnSnO}_{3}$ Cubes with Controllable Shells Enabling Highly Efficient Chemical Sensing Detection of Formaldehyde Vapors, ACS Appl. Mater. Interfaces, 2017, 9, 14525-14533.

90 S.-L. Xiong and H.-C. Zeng, Serial Ionic Exchange for the Synthesis of Multishelled Copper Sulfide Hollow Spheres, Angew. Chem., Int. Ed., 2012, 51, 949-952.

91 D.-Y. Xue, F.-F. Xue, X.-P. Lin, F.-Y. Zong, J.-M. Zhang and Q.-H. Li, Coordination Polymer Derived General Synthesis of Multi-shelled Hollow Metal Oxides for Lithium-ion Batteries, Nanoscale, 2019, 11, 17478-17484.

92 L. Liu, J.-J. Shi, R.-Y. Wang, H.-X. Cao and Z.-W. Liu, Fabrication of Double-shelled $\mathrm{Fe}_{2} \mathrm{O}_{3} / \mathrm{CeO}_{2}$ Boxes from $\mathrm{CeO}_{2}$-modified Prussian Blue and Their Enhanced Performances for CO Removal and Water Treatment, J. Alloys Compd., 2017, 725, 544-556.

93 X.-Z. Song, L. Qiao, K.-M. Sun, Z.-Q. Tan, W. Ma, X.-L. Kan, F.-F. Sun, T. Huang and X.-F. Wang, Triple-shelled $\mathrm{ZnO} / \mathrm{ZnFe}_{2} \mathrm{O}_{4}$ Heterojunctional Hollow Microspheres Derived from Prussian Blue Analogue as High-performance Acetone Sensors, Sens. Actuators, B, 2018, 256, 374-382.

94 W.-X. Liu, J.-J. Huang, Q. Yang, S.-J. Wang, X.-M. Sun, W.-N. Zhang, J.-F. Liu and F.-W. Huo, Multi-shelled Hollow Metal-Organic Frameworks, Angew. Chem., Int. Ed., 2017, 56, 5512-5516.

95 D. Y. Hong, Y. K. Hwang, C. Serre, G. Ferey and J. S. Chang, Porous Chromium Terephthalate MIL-101 with Coordinatively Unsaturated Sites: Surface Functionalization,
Encapsulation, Sorption and Catalysis, Adv. Funct. Mater., 2009, 19, 1537-1552.

96 G. Ferey, C. Mellot-Draznieks, C. Serre, F. Millange, J. Dutour, S. Surble and I. Margiolaki, A Chromium TerephthalateBased Solid with Unusually Large Pore Volumes and Surface Area, Science, 2005, 309, 2040-2042.

97 W. Cho, Y. H. Lee, H. J. Lee and M. Oh, Multi Ball-in-ball Hybrid Metal Oxides, Adv. Mater., 2011, 23, 1720-1723.

98 P. Zhang, B.-Y. Guan, L. Yu and X.-W. Lou, Facile Synthesis of Multi-shelled ZnS-CdS Cages with Enhanced Photoelectrochemical Performance for Solar Energy Conversion, Chem, 2018, 4, 162-173.

99 B.-Y. Guan, A. Kushima, L. Yu, S. Li, J. Li and X.-W. Lou, Coordination Polymers Derived General Synthesis of Multishelled Mixed Metal-Oxide Particles for Hybrid Supercapacitors, Adv. Mater., 2017, 29, 1605902.

100 B.-Y. Guan, L. Yu and X.-W. Lou, General Synthesis of Multishell Mixed-Metal Oxyphosphide Particles with Enhanced Electrocatalytic Activity in the Oxygen Evolution Reaction, Angew. Chem., Int. Ed., 2017, 56, 2386-2389.

101 H. Hu, B.-Y. Guan, B.-Y. Xia and X.-W. Lou, Designed Formation of $\mathrm{Co}_{3} \mathrm{O}_{4} / \mathrm{NiCo}_{2} \mathrm{O}_{4}$ Double-Shelled Nanocages with Enhanced Pseudocapacitive and Electrocatalytic Properties, J. Am. Chem. Soc., 2015, 137, 5590-5595.

102 H. Hu, L. Han, M.-Z. Yu, Z.-Y. Wang and X.-W. Lou, Metalorganic-framework-engaged Formation of Co Nanoparticleembedded Carbon@ $\mathrm{Co}_{9} \mathrm{~S}_{8}$ Double-shelled Nanocages for Efficient Oxygen Reduction, Energy Environ. Sci., 2016, 9, 107-111.

103 L.-F. Shen, L. Yu, X.-Y. Yu, X.-G. Zhang and X.-W. Lou, SelfTemplated Formation of Uniform $\mathrm{NiCo}_{2} \mathrm{O}_{4}$ Hollow Spheres with Complex Interior Structures for Lithium-ion Batteries and Supercapacitors, Angew. Chem., Int. Ed., 2015, 54, 1868-1872.

104 G.-Q. Zhang, L. Yu, H.-B. Wu, H. E. Hoster and X.-W. Lou, Formation of $\mathrm{ZnMn}_{2} \mathrm{O}_{4}$ Ball-in-Ball Hollow Microspheres as a High-Performance Anode for Lithium-ion Batteries, Adv. Mater., 2012, 24, 4609-4613.

105 J.-F. Li, J.-Z. Wang, X. Liang, Z.-J. Zhang, H.-K. Liu, Y.-T. Qian and S.-L. Xiong, Hollow $\mathrm{MnCo}_{2} \mathrm{O}_{4}$ Submicrospheres with Multilevel Interiors: From Mesoporous Spheres to Yolk-in-double-shell Structures, ACS Appl. Mater. Interfaces, 2014, 6, 24-30.

106 L.-F. Shen, L. Yu, H.-B. Wu, X.-Y. Yu, X.-G. Zhang and X.-W. Lou, Formation of Nickel Cobalt Sulfide Ball-in-ball Hollow Spheres with Enhanced Electrochemical Pseudocapacitive Properties, Nat. Commun., 2015, 6, 6694.

107 Y. Lu, J.-W. Nai and X.-W. Lou, Formation of $\mathrm{NiCo}_{2} \mathrm{~V}_{2} \mathrm{O}_{8}$ Yolk-double Shell Spheres with Enhanced Lithium Storage Properties, Angew. Chem., Int. Ed., 2018, 57, 2899-2903.

108 Y.-W. Wang, L. Yu and X.-W. Lou, Formation of Triple-Shelled Molybdenum-Polydopamine Hollow Spheres and Their Conversion into $\mathrm{MoO}_{2}$ /Carbon Composite Hollow Spheres for Lithium-Ion Batteries, Angew. Chem., 2016, 128, 14888-14892.

109 J.-X. Guo, X.-Q. Zhang, Y.-F. Sun, X.-H. Zhang, L. Tang and X. Zhang, Double-shell CuS Nanocages as Advanced 
Supercapacitor Electrode Materials, J. Power Sources, 2017, 355, 31-35.

110 C.-Z. Wu, X.-D. Zhang, B. Ning, J.-L. Yang and Y. Xie, Shape Evolution of New-phased Lepidocrocite $\mathrm{VOOH}$ from SingleShelled to Double-Shelled Hollow Nanospheres on the Basis of Programmed Reaction-Temperature Strategy, Inorg. Chem., 2009, 48, 6044-6054.

111 H. B. Lin, H. B. Rong, W. Z. Huang, Y. H. Liao, L. D. Xing, M. Q. Xu, X. P. Li and W. S. Li, Triple-Shelled $\mathrm{Mn}_{2} \mathrm{O}_{3}$ Hollow Nanocubes: Forceinduced Synthesis and Excellent Performance as the Anode in Lithium-Ion Batteries, J. Mater. Chem. A, 2014, 2, 14189-14194.

112 H. Zhao, J.-F. Chen, Y. Zhao, L. Jiang, J.-W. Sun and J. Yun, Hierarchical Assembly of Multilayered Hollow Microspheres from an Amphiphilic Pharmaceutical Molecule of Azithromycin, Adv. Mater., 2008, 20, 3682-3686.

113 A.-Q. Pan, H.-B. Wu, L. Yu and X.-W. Lou, Template-Free Synthesis of $\mathrm{VO}_{2}$ Hollow Microspheres with Various Interiors and Their Conversion into $\mathrm{V}_{2} \mathrm{O}_{5}$ for Lithium-Ion Batteries, Angew. Chem., Int. Ed., 2013, 52, 2226-2230.

114 L. Zhou, H.-Y. Xu, H.-W. Zhang, J. Yang, S. B. Hartono, K. Qian, J. Zou and C.-Z. Yu, Cheap and Scalable Synthesis of $\alpha-\mathrm{Fe}_{2} \mathrm{O}_{3}$ Multi-shelled Hollow Spheres as Highperformance Anode Materials for Lithium Ion Batteries, Chem. Commun., 2013, 49, 8695-8697.

115 Z. Padashbarmchi, A. H. Hamidian, H.-W. Zhang, L. Zhou, N. Khorasani, M. Kazemzad and C.-Z. Yu, A Systematic Study on the Synthesis of $\alpha-\mathrm{Fe}_{2} \mathrm{O}_{3}$ Multi-shelled Hollow Spheres, RSC Adv., 2015, 5, 10304-10309.

116 G. D. Park, J. H. Lee, J. K. Lee and Y. C. Kang, Effect of Esterification Reaction of Citric Acid and Ethylene Glycol on the Formation of Multi-shelled Cobalt Oxide Powders with Superior Electrochemical Properties, Nano Res., 2014, 7, 1738-1748.

117 J. S. M. Zanjani, B. S. Okan, I. Letofsky-Papst, M. Yildiz and Y. Z. Menceloglu, Rational Design and Direct Fabrication of Multi-Walled Hollow Electrospun Fibers with Controllable Structure and Surface Properties, Eur. Polym. J., 2015, 62, 66-76.

118 G. Zhang, B. Y. Xia, C. Xiao, L. Yu, X. Wang, Y. Xie and X. W. Lou, General Formation of Complex Tubular Nanostructures of Metal Oxides for the Oxygen Reduction Reaction and Lithium-Ion Batteries, Angew. Chem., Int. Ed., 2013, 52, 8643-8647.

119 L. Zhang and H. Wang, Interior Structural Tailoring of $\mathrm{Cu}_{2} \mathrm{O}$ Shell-in-shell Nanostructures through Multistep Ostwald Ripening, J. Phys. Chem. C, 2011, 115, 18479-18485.

120 C. C. Yec and H. C. Zeng, Synthetic Architecture of Multiple Core-Shell and Yolk-shell Structures of $\left(\mathrm{Cu}_{2} \mathrm{O} @\right) \mathrm{nCu}_{2} \mathrm{O}$ ( $n=1-4)$ with Centricity and Eccentricity, Chem. Mater., 2012, 24, 1917-1929.

$121 \mathrm{~J}$. Li and H.-C. Zeng, Size Tuning, Functionalization, and Reactivation of $\mathrm{Au}$ in $\mathrm{TiO}_{2}$ Nanoreactors, Angew. Chem., Int. Ed., 2005, 44, 4342-4345.

122 B. Liao, Z.-G. An and J.-J. Zhang, Highly Efficient Largescale Preparation and Electromagnetic Property Control of
Silica-NiFeP Double Shell Composite Hollow Particles, RSC Adv., 2017, 7, 21721-21732.

123 A. S. Arico, P. Bruce, B. Scrosati, J.-M. Tarascon and W. V. Schalkwijk, Nanostructured Materials for Advanced Energy Conversion and Storage Devices, Nat. Mater., 2005, 4, 366-377.

124 J. R. Miller and P. Simon, Electrochemical Capacitors for Energy Management, Science, 2008, 321, 651-652.

125 J.-X. Zhu, D. Yang, Z.-Y. Yin, Q.-Y. Yan and H. Zhang, Graphene and Graphene-Based Materials for Energy Storage Applications, Small, 2014, 10, 3480-3498.

126 J. B. Goodenough, Electrochemical Energy Storage in a Sustainable Modern Society, Energy Environ. Sci., 2014, 7, 14-18.

127 G.-P. Wang, L. Zhang and J.-J. Zhang, A Review of Electrode Materials for Electrochemical Supercapacitors, Chem. Soc. Rev., 2012, 41, 797-828.

128 Q. Chen, W. Wei, J. Tang, J. Lin, S. Li and M. Zhu, Dopamine-Assisted Preparation of $\mathrm{Fe}_{3} \mathrm{O}_{4} @ \mathrm{MnO}_{2}$ Yolk@Shell Microspheres for Improved Pseudocapacitive Performance, Electrochim. Acta, 2019, 317, 628-637.

129 L. Kong, Q. Ma, Z. Xu, X. Shen, J. Wang and J. Zhu, ThreeDimensional Graphene Network Deposited with Mesoporous Nitrogen-Doped Carbon from Non-Solvent Induced Phase Inversion for High-Performance Supercapacitors, J. Colloid Interface Sci., 2020, 558, 21-31.

130 S. Li, K. Yang, P. Ye, K. Ma, Z. Zhang and Q. Huang, ThreeDimensional Porous Carbon $/ \mathrm{Co}_{3} \mathrm{O}_{4}$ Composites Derived from Graphene/Co-MOF for High Performance Supercapacitor Electrodes, Appl. Surf. Sci., 2020, 503, 144090.

131 M. Zhu, Q. Chen, W. Tong, J. Kan and W. Sheng, Preparation and Application of $\mathrm{Fe}_{3} \mathrm{O}_{4}$ Nanomaterials, Prog. Chem., 2017, 29, 1366-1394.

132 M. Sevilla and R. Mokaya, Energy Storage Applications of Activated Carbons: Supercapacitors and Hydrogen Storage, Energy Environ. Sci., 2014, 7, 1250-1280.

133 W.-F. Wei, X.-W. Cui, W.-X. Chen and D. G. Ivey, Manganese Oxide-based Materials as Electrochemical Supercapacitor Electrodes, Chem. Soc. Rev., 2011, 40, 1697-1721.

134 C.-Z. Yuan, H.-B. Wu, Y. Xie and X.-W. Lou, Mixed Transitionmetal Oxides: Design, Synthesis, and Energy-related Applications, Angew. Chem., Int. Ed., 2014, 53, 1488-1504.

135 K. Wang, H.-P. Wu, Y.-N. Meng and Z.-X. Wei, Conducting Polymer Nanowire Arrays for High Performance Supercapacitors, Small, 2013, 10, 14-31.

136 M. Zhu, Q. Chen, J. Tang, W. Wei and S. Li, Core@Shell $\beta$-FeOOH@Polypyrolle Derived N, S-codoped $\mathrm{Fe}_{3} \mathrm{O}_{4} @ \mathrm{~N}$ Doped Porous Carbon Nanococoons for High Performance Supercapacitors, Appl. Surf. Sci., 2019, 480, 582-592.

137 M. Zhu, Q. Chen, J. Kan, J. Tang, W. Wei, J. Lin and S. Li, Coblat Oxide Nanoparticles Embedded in N-Doped Porous Carbon as Efficient Electrode for Supercapacitor, Energy Technol., 2019, 1800963.

138 M. Zhu, J. Kan, J. Pan, W. Tong, Q. Chen, J. Wang and S. Li, One-Pot Hydrothermal Fabrication of $\alpha-\mathrm{Fe}_{2} \mathrm{O}_{3} @ \mathrm{C}$ Nanocomposites for Electrochemical Energy Storage, J. Energy Chem., 2019, 28, 1-8. 
139 K. Zhang, Z. Shang, S. Wu, J. Wang, W. Sheng, X. Shen and M. Zhu, Commercialized Benzoxazine Resin-Derived Porous Carbon as high Performance Electrode Materials for Supercapacitor, J. Inorg. Organomet. Polym., 2017, 27, 1423-1429.

140 X. Yuan, X. Yan, C. Zhou, J. Wang, D. Wang, H. Jiang, Y. Zhu, X. Tao and X. Cheng, Decorating Carbon Nanosheets with Copper Oxide Nanoparticles for Boosting the Electrochemical Performance of Symmetric Coin Cell Supercapacitor with Different Electrolytes, Ceram. Int., 2020, 46, 435-443.

141 X. Yang, J. Kan, F. Zhang, M. Zhu and S. Li, Facile Fabrication of $\mathrm{Mn}^{2+}$ Doped Magnetite Microspheres as Efficient Electrode Material for Supercapacitors., J. Inorg. Organomet. Polym., 2017, 27, 542-551.

142 S. Hussain, P. Wan, N. Aslam, G. Qiao, G. Liu and M. Wang, Ag-Doped NiO Porous Network Structure on Ni Foam as Electrode for Supercapacitors, J. Mater. Sci.: Mater. Electron., 2018, 29, 1759-1765.

143 Y. Wang, M. Zhang, Y. Li, T. Ma, H. Liu, D. Pan, X. Wang and A. Wang, Rational Design 3D Nitrogen Doped Graphene Supported Spatial Crosslinked $\mathrm{Co}_{3} \mathrm{O}_{4} @ \mathrm{NiCo}_{2} \mathrm{O}_{4}$ on Nickel Foam for Binder-Free Supercapacitor Electrodes, Electrochim. Acta, 2018, 290, 12-20.

144 M. Zhang, Y. Wang, D. Pan, Y. Li, Z. Yan and J. Xie, Nitrogen-Doped 3D Graphene/MWNTs NanoframeworkEmbedded $\mathrm{Co}_{3} \mathrm{O}_{4}$ for High Electrochemical Performance Supercapacitors, ACS Sustainable Chem. Eng., 2017, 5, 5099-5107.

145 X. Cai, H. Bai, Y. Liu and W. Shi, Facile In Situ Synthesis of $\mathrm{Ag}$ and $\mathrm{MnO}_{2}$ Anchored on Carbon Microtubes for High-Performance Asymmetric Supercapacitor Applications. Appl, Mater. Today, 2018, 11, 193-199.

146 H. Sun, J. Pan, X. Yan, W. Shen, W. Zhong and X. Cheng, $\mathrm{MnO}_{2}$ Nanoneedles Loaded on Silicon Oxycarbide-Derived Hierarchically Porous Carbon for Supercapacitor Electrodes with Enhanced Electrochemical Performance, Ceram. Int., 2019, 45, 24802-24810.

147 Y. Liu, X. Cai, B. Luo, M. Yan, J. Jiang and W. Shi, $\mathrm{MnO}_{2}$ Decorated on Carbon Sphere Intercalated Graphene Film for High-Performance Supercapacitor Electrodes, Carbon, 2016, 107, 426-432.

148 Z.-H. Yang, F.-F. Xu, W.-X. Zhang, Z.-S. Mei, B. Pei and X. Zhu, Controllable Preparation of Multishelled NiO Hollow Nanospheres via Layer-by-layer Self-assembly for Supercapacitor Application, J. Power Sources, 2014, 246, 24-31.

149 H.-L. Wang, Y. Yang, Y.-Y. Liang, G.-Y. Zheng, Y.-G. Li, Y. Cui and H.-J. Dai, Rechargeable $\mathrm{Li}^{-\mathrm{O}_{2}}$ Batteries with a Covalently Coupled $\mathrm{MnCo}_{2} \mathrm{O}_{4}$-graphene Hybrid as an Oxygen Cathode Catalyst, Energy Environ. Sci., 2012, 5, 7931-7935.

150 C. Huang, Y. Ding, C. Hao, S. Zhou, X. Wang, H. Gao, L. Zhu and J. Wu, PVP-Assisted Growth of Ni-Co Oxide on N-Doped Reduced Graphene Oxide with Enhanced Pseudocapacitive Behavior, Chem. Eng. J., 2019, 378, 122202.
151 M. Zhu, X. Zhang, Y. Zhou, C. Zhuo, J. Huang and S. Li, Facile Solvothermal Synthesis of Porous $\mathrm{ZnFe}_{2} \mathrm{O}_{4}$ Microspheres for Capacitive Pseudocapacitors, RSC Adv., 2015, 5, 39270-39277.

152 S. E. Moosavifard, S. K. Kaverlavani, J. Shamsi and A. Bakouei, Hierarchical Multi-shelled Nanoporous Mixed Copper Cobalt Phosphide Hollow Microspheres as a Novel Advanced Electrode for High-performance Asymmetric Supercapacitors, J. Mater. Chem. A, 2017, 5, 18429-18433.

153 A. A. Ensafi, S. E. Moosavifard, B. Rezaei and S. K. Kaverlavani, Engineering Onion-like Nanoporous $\mathrm{CuCo}_{2} \mathrm{O}_{4}$ Hollow Spheres Derived from Bimetal-organic Frameworks for High-performance Asymmetric Supercapacitors, J. Mater. Chem. A, 2018, 6, 10497-10506.

154 H. Niu, Y. Liu, B. Mao, N. Xin, H. Jia and W. Shi, In-Situ Embedding MOFs-Derived Copper Sulfide Polyhedrons in Carbon Nanotube Networks for Hybrid Supercapacitor with Superior Energy Density, Electrochim. Acta, 2020, 329, 135130.

155 X.-F. Lu, D.-J. Wu, R.-Z. Li, Q. Li, S.-H. Ye, Y.-X. Tong and G.-R. Li, Hierarchical $\mathrm{NiCo}_{2} \mathrm{O}_{4}$ Nanosheets@Hollow Microrod Arrays for High-performance Symmetric Supercapacitors, J. Mater. Chem. A, 2014, 2, 4706-4713.

156 D. P. Dubal, G. S. Gund, C. D. Lokhande and R. Holze, Controlled Growth of CoSx Nanostrip Arrays (CoSx-NSA) on Nickel Foam for Asymmetric Supercapacitors, Energy Technol., 2014, 2, 401-408.

157 H.-B. Li, M.-H. Yu, F.-X. Wang, P. Liu, Y. Liang, J. Xiao, C.-X. Wang, Y.-X. Tong and G.-W. Yang, Amorphous Nickel Hydroxide Nanospheres with Ultrahigh Capacitance and Energy Density as Electrochemical Pseudocapacitor Materials, Nat. Commun., 2013, 4, 1894.

158 X.-Z. Yu, B.-G. Lu and Z. Xu, Super Long-Life Supercapacitors Based on the Construction of Nanohoneycomb-like Strongly Coupled $\mathrm{CoMoO}_{4}-3 \mathrm{D}$ Graphene Hybrid Electrodes, Adv. Mater., 2014, 26, 1044-1051.

159 C.-Z. Wei, Q.-L. Ru, X.-T. Kang, H.-Y. Hou, C. Cheng and D.-J. Zhang, Self-template Synthesis of Double Shelled $\mathrm{ZnS}-\mathrm{NiS}_{1.97}$ Hollow Spheres for Electrochemical Energy Storage, Appl. Surf. Sci., 2018, 435, 993-1001.

160 Y.-P. Wang, T. Zhu, Y.-F. Zhang, X.-Z. Kong, S.-Q. Liang, G.-Z. Cao and A.-Q. Pan, Rational Design of Multi-shelled $\mathrm{CoO} / \mathrm{Co}_{9} \mathrm{~S}_{8}$ Hollow Microspheres for High-performance Hybrid Supercapacitors, J. Mater. Chem. A, 2017, 5, 18448-18456.

161 Y.-P. Wang, A.-Q. Pan, Y.-F. Zhang, J.-R. Shi, J.-D. Lin, S.-Q. Liang and G.-Z. Cao, Heterogeneous NiS/NiO Multi-shelled Hollow Microspheres with Enhanced Electrochemical Performances for Hybrid-type Asymmetric Supercapacitors, J. Mater. Chem. A, 2018, 6, 9153-9160.

162 M.-M. Fang, Z.-M. Chen, Y. Liu, W. Liu and Q. Xu, Uniform Discrete Nitrogen-Doped Double-Shelled Cage-like Hollow Carbon Spheres with Direct Large Mesopores for HighPerformance Supercapacitors, Energy Technol., 2017, 5, 2198-2204.

163 J. B. Goodenough and Y. Kim, Challenges for Rechargeable Li Batteries, Chem. Mater., 2009, 22, 587-603. 
164 X.-M. Ma, Z.-P. Wei, H.-J. Han, X.-B. Wang, K.-Q. Cui and L. Yang, Tunable Construction of Multi-shell Hollow $\mathrm{SiO}_{2}$ Microspheres with Hierarchically Porous Structure as High-performance Anodes for Lithium-ion Batteries, Chem. Eng. J., 2017, 323, 252-259.

165 Y.-F. Yuan, L.-W. Ye, D. Zhang, F. Chen, M. Zhu, L.-N. Wang, S.-M. Yin, G.-S. Cai and S.-Y. Guo, $\mathrm{NiCo}_{2} \mathrm{~S}_{4}$ Multishelled Hollow Polyhedrons as High-performance Anode Materials for Lithium-ion Batteries, Electrochim. Acta, 2019, 299, 289-297.

166 M. V. Reddy, G. V. S. Rao and B. V. R. Chowdari, Metal Oxides and Oxysalts as Anode Materials for $\mathrm{Li}$ Ion Batteries, Chem. Rev., 2013, 113, 5364-5457.

167 J.-Y. Wang, N.-L. Yang, H.-J. Tang, Z.-H. Dong, Q. Jin, M. Yang, D. Kisailus, H.-J. Zhao, Z.-Y. Tang and D. Wang, Accurate Control of Multishelled $\mathrm{Co}_{3} \mathrm{O}_{4}$ Hollow Microspheres as High-Performance Anode Materials in LithiumIon Batteries, Angew. Chem., Int. Ed., 2013, 52, 6417-6420.

168 Z.-G. Wu, Y.-J. Zhong, J.-T. Li, X.-D. Guo, L. Huang, B.-H. Zhong and S.-G. Sun, l-Histidine-assisted Templatefree Hydrothermal Synthesis of $\alpha-\mathrm{Fe}_{2} \mathrm{O}_{3}$ Porous Multishelled Hollow Spheres with Enhanced Lithium Storage Properties, J. Mater. Chem. A, 2014, 2, 12361-12367.

169 J.-Z. Yin, Y. Zhang, Q.-Y. Lu, X.-L. Wu, Z.-J. Jiang, L.-Y. Dang, H.-F. Ma, Y.-Y. Guo, F. Gao and Q.-Y. Yan, Unable $\mathrm{Co}_{3} \mathrm{O}_{4}$ Hollow Structures (from Yolk-shell to Multi-shell) and their Li Storage Properties, J. Mater. Chem. A, 2017, 5, 12757-12761.

170 Y. Lu, L. Yu, M.-H. Wu, Y. Wang and X.-W. Lou, Construction of Complex $\mathrm{Co}_{3} \mathrm{O}_{4} @ \mathrm{Co}_{3} \mathrm{~V}_{2} \mathrm{O}_{8}$ Hollow Structures from Metal-Organic Frameworks with Enhanced Lithium Storage Properties, Adv. Mater., 2018, 30, 1702875.

171 P. Poizot, S. Laruelle, S. Grugeon, L. Dupont and J.-M. Tarascon, Nano-sized Transition-metal Oxides as Negativeelectrode Materials for Lithium-ion Batteries, Nature, 2000, 407, 496-499.

172 J. Jiang, Y.-Y. Li, J.-P. Liu, X.-T. Huang, C.-Z. Yuan and X.-W. Lou, Recent Advances in Metal Oxide-based Electrode Architecture Design for Electrochemical Energy Storage, Adv. Mater., 2012, 24, 5166-5180.

173 H. Li, H.-R. Ma, M. Yang, B. Wang, H. Shao, L. Wang, R.-B. Yu and D. Wang, Highly Controlled Synthesis of Multi-shelled NiO Hollow Microspheres for Enhanced Lithium Storage Properties, Mater. Res. Bull., 2017, 87, 224-229.

174 L. Liu, Q. Liu, W. Zhao, G. Li, L. Wang, W. Shi and L. Chen, Enhanced Electrochemical Performance of Orientated $\mathrm{VO}_{2}$ (B) Raft-Like Nanobelt Arrays Through Direct Lithiation for Lithium Ion Batteries, Nanotechnology, 2017, 28, 065404.

175 Y.-P. Wang, Z.-W. Nie, A.-Q. Pan, Y.-F. Zhang, X.-Z. Kong, T. Zhu, S.-Q. Liang and G.-Z. Cao, Self-templating Synthesis of Double-wall Shelled Vanadium Oxide Hollow Microspheres for High-performance Lithium Ion Batteries, J. Mater. Chem. A, 2018, 6, 6792-6799.

176 F.-F. Guo, M.-H. Fan, P.-P. Jin, H. Chen, Y.-Y. Wu, G.-D. $\mathrm{Li}$ and X.-X. Zou, Precursor-mediated Synthesis of
Double-shelled $\mathrm{V}_{2} \mathrm{O}_{5}$ Hollow Nanospheres as Cathode Material for Lithium-ion Batteries, CrystEngComm, 2016, 18, 4068-4073.

177 G.-L. Wu, Z.-R. Jia, Y.-H. Cheng, H.-X. Zhang, X.-F. Zhou and H.-J. Wu, Easy Synthesis of Multi-shelled ZnO Hollow Spheres and their Conversion into Hedgehog-like ZnO Hollow Spheres with Superior Rate Performance for Lithium Ion Batteries, Appl. Surf. Sci., 2019, 464, 472-478.

178 S.-H. Qu, Y.-K. Yu, K.-J. Lin, P.-Y. Liu, C.-H. Zheng, L.-D. Wang, T.-T. Xu, Z.-D. Wang and H.-J. Wu, Easy Hydrothermal Synthesis of Multi-shelled $\mathrm{La}_{2} \mathrm{O}_{3}$ Hollow Spheres for Lithium-ion Batteries, J. Mater. Sci.: Mater. Electron., 2018, 29, 1232-1237.

179 P. Dou, Z.-Z. Cao, C. Wang, J. Zheng and X.-H. Xu, Multilayer $\mathrm{Zn}$-doped $\mathrm{SnO}_{2}$ Hollow Nanospheres Encapsulated in Covalently Interconnected Three-dimensional Graphene Foams for High Performance Lithium-ion Batteries, Chem. Eng. J., 2017, 320, 405-415.

180 G.-L. Wu, H.-J. Wu, K.-K. Wang, C.-H. Zheng, Y.-Q. Wang and A.-L. Feng, Facile Synthesis and Application of Multishelled $\mathrm{SnO}_{2}$ Hollow Spheres in Lithium Ion Battery, RSC Adv., 2016, 6, 58069-58076.

181 M.-J. Zhou, Y.-C. Liu, J. Chen and X.-L. Yang, Double Shelled Hollow $\mathrm{SnO}_{2}$ /polymer Microsphere as a Highcapacity Anode Material for Superior Reversible Lithium Ion Storage, J. Mater. Chem. A, 2015, 3, 1068-1076.

182 J. Zhang, J.-W. Wan, J.-Y. Wang, H. Ren, R.-B. Yu, L. Gu, Y.-L. Liu, S.-H. Feng and D. Wang, Hollow Multi-shelled Structure with Metal-organic-framework-derived Coatings for Enhanced Lithium Storage, Angew. Chem., Int. Ed., 2019, 58, 5266-5271.

183 G.-F. Li, S.-Q. Liu, Y. Pan, T.-Y. Zhou, J.-D. Ding, Y.-M. Sun and Y.-Q. Wang, Self-templated Formation of $\mathrm{CuCo}_{2} \mathrm{O}_{4}$ Triple-shelled Hollow Microspheres for All-solid-state Asymmetric Supercapacitors, J. Alloys Compd., 2019, 787, 694-699.

184 C.-W. Jiao, Z.-M. Wang, X.-X. Zhao, H. Wang, J. Wang, R.-B. Yu and D. Wang, Triple-Shelled Manganese-cobalt Oxide Hollow Dodecahedra with Highly Enhanced Performance for Rechargeable Alkaline Batteries, Angew. Chem., Int. Ed., 2019, 58, 1008-1013.

185 J.-Y. Xu, H. Zhang, R.-F. Wang, P.-B. Xu, Y.-L. Tong, Q.-Y. Lu and F. Gao, Delicate Control of Multishelled Zn-Mn-O Hollow Microspheres as a High-Performance Anode for Lithium-Ion Batteries, Langmuir, 2018, 34, 1242-1248.

186 D.-W. Li, X.-X. Zhao, R.-B. Yu, B. Wang, H. Wang and D. Wang, Formation of Multi-shelled Nickel-based Sulfide Hollow Spheres for Rechargeable Alkaline Batteries, Inorg. Chem. Front., 2018, 5, 535-540.

187 Y. Fang, Y.-Y. Lv, R.-C. Che, H.-Y. Wu, X.-H. Zhang, D. Gu, G.-F. Zheng and D.-Y. Zhao, Two-Dimensional Mesoporous Carbon Nanosheets and Their Derived Graphene Nanosheets: Synthesis and Efficient Lithium Ion Storage, J. Am. Chem. Soc., 2013, 135, 1524-1530.

188 Z.-S. Wu, W.-C. Ren, L. Xu, F. Li and H.-M. Cheng, Doped Graphene Sheets As Anode Materials with Superhigh Rate 
and Large Capacity for Lithium Ion Batteries, ACS Nano, 2011, 5, 5463-5471.

189 D. Zhang, G. Wang, L. Xu, J. Lian, J. Bao, Y. Zhao, J. Qiu and H. Li, Defect-Rich N-Doped Porous Carbon Derived from Soybean for High Rate Lithium-Ion Batteries, Appl. Surf. Sci., 2018, 451, 298-305.

190 Y.-Z. Zhang, X.-L. Zong, L. Zhan, X.-Y. Yu, J. Gao, C.-C. Xun, P.-Y. Li and Y.-L. Wang, Double-shelled Hollow Carbon Sphere with Microporous Outer Shell towards High Performance Lithium-sulfur Battery, Electrochim. Acta, 2018, 284, 89-97.

191 X.-F. Niu, Y.-F. Li, Y.-J. Hu, H. Jiang, X.-Y. Hou, W.-G. Li, S.-J. Qiu and C.-Z. Li, Aerosol Construction of Multi-shelled $\mathrm{LiMn}_{2} \mathrm{O}_{4}$ Hollow Microspheres as a Cathode in Lithium Ion Batteries, New J. Chem., 2016, 40, 1839-1844.

192 F. Wang, J.-Y. Wang, H. Ren, H.-J. Tang, R.-B. Yu and D. Wang, Multi-shelled $\mathrm{LiMn}_{2} \mathrm{O}_{4}$ Hollow Microspheres as Superior Cathode Materials for Lithium-ion Batteries, Inorg. Chem. Front., 2016, 3, 365-369.

193 M. D. Slater, D. H. Kim, E. Lee and C. S. Johnson, Sodiumion Batteries, Adv. Funct. Mater., 2012, 23, 947-958.

194 V. Palomares, P. Serras, I. Villaluenga, K. B. Hueso, J. Carretero-González and T. Rojo, Na-ion Batteries, Recent Advances and Present Challenges to Become Low Cost Energy Storage Systems, Energy Environ. Sci., 2012, 5, 5884-5901.

195 X. Zhang, Y.-P. Zhou, B. Luo, H.-C. Zhu, W. Chu and K. Huang, Microwave-Assisted Synthesis of $\mathrm{NiCo}_{2} \mathrm{O}_{4}$ Double-Shelled Hollow Spheres for High-performance Sodium Ion Batteries, Nano-Micro Lett., 2018, 10, 13.

196 F. Xie, L. Zhang, Q. Gu, D. Chao, M. Jaroniec and S. Z. Qiao, Multi-Shell Hollow Structured $\mathrm{Sb}_{2} \mathrm{~S}_{3}$ for Sodium-Ion Batteries with Enhanced Energy Density, Nano Energy, 2019, 60, 591-599.

197 S. Huang, C. Meng, M. Xiao, S. Ren, S.-J. Wang, D.-M. Han, Y.-N. Li and Y.-Z. Meng, Multi-shell Tin Phosphide Nanospheres as High Performance Anode Material for a Sodium Ion Battery, Sustain, Energy Fuels, 2017, 1, 1944-1949.

198 E. M. Salhabi, J.-L. Zhao, J.-Y. Wang, M. Yang, B. Wang and D. Wang, Hollow Multi-shelled Structural $\mathrm{TiO}_{2-x}$ with Multiple Spatial Confinement for Long-life Lithiumsulfur Batteries, Angew. Chem., 2019, 58, 9078-9082.

199 X.-X. Chen, X.-Y. Ding, C.-S. Wang, Z.-Y. Feng, L.-Q. Xu, X. Gao, Y.-J. Zhai and D.-B. Wang, A Multi-shelled CoP Nanosphere Modified Separator for Highly Efficient Li-S Batteries, Nanoscale, 2018, 10, 13694-13701.

200 S.-Q. Chen, X.-D. Huang, B. Sun, J.-Q. Zhang, H. Liu and G.-X. Wang, Multi-shelled Hollow Carbon Nanospheres for Lithium-sulfur Batteries with Superior Performances, J. Mater. Chem. A, 2014, 2, 16199-16207.

201 J.-W. Gong, K. Sumathy, Q.-Q. Qiao and Z.-P. Zhou, Review on Dye-sensitized Solar Cells (DSSCs): Advanced Techniques and Research Trends, Renewable Sustainable Energy Rev., 2017, 68, 234-246.

202 W.-Q. Wu, Y.-F. Xu, H.-S. Rao, C.-Y. Su and D.-B. Kuang, Multistack Integration of Three-Dimensional Hyperbranched
Anatase Titania Architectures for High-Efficiency DyeSensitized Solar Cells, J. Am. Chem. Soc., 2014, 136, 6437-6445. 203 M. Jafarzadeh, C. S. Sipaut, J. Dayou and R. F. Mansa, Recent Progresses in Solar Cells: Insight into Hollow Micro/nano-Structures, Renewable Sustainable Energy Rev., 2016, 64, 543-568.

204 H.-Q. Wang, M. Miyauchi, Y. Ishikawa, A. Pyatenko, N. Koshizaki, Y. Li, L. Li, X.-Y. Li, Y. Bando and D. Golberg, Single-Crystalline Rutile $\mathrm{TiO}_{2}$ Hollow Spheres: RoomTemperature Synthesis, Tailored Visible-Light-Extinction, and Effective Scattering Layer for Quantum Dot-Sensitized Solar Cells, J. Am. Chem. Soc., 2011, 133, 19102-19109.

205 L. P. Heiniger, F. Giordano, T. Moehl and M. Graetzel, Mesoporous $\mathrm{TiO}_{2}$ Beads Offer Improved Mass Transport for Cobalt-Based Redox Couples Leading to High Efficiency Dye-Sensitized Solar Cells, Adv. Energy Mater., 2014, 4, 1400168.

206 J. H. Pan, X. Z. Wang, Q.-Z. Huang, C. Shen, Z. Y. Koh, Q. Wang, A. Engel and D. W. Bahnemann, Large-scale Synthesis of Urchin-like Mesoporous $\mathrm{TiO}_{2}$ Hollow Spheres by Targeted Etching and Their Photoelectrochemical Properties, Adv. Funct. Mater., 2014, 24, 95-104.

207 M. S. Ahmad, A. K. Pandey and N. A. Rahim, Advancements in the Development of $\mathrm{TiO}_{2}$ Photoanodes and its Fabrication Methods for Dye Sensitized Solar Cell (DSSC) Applications, Renewable Sustainable Energy Rev., 2017, 77, 89-108.

208 X.-P. Lin, D.-M. Song, X.-Q. Gu, Y.-L. Zhao and Y. H. Qiang, Synthesis of Hollow Spherical $\mathrm{TiO}_{2}$ for Dye-sensitized Solar Cells with Enhanced Performance, Appl. Surf. Sci., 2012, 263, 816-820.

209 X. Wu, G.-Q. Lu and L.-Z. Wang, Shell-in-Shell $\mathrm{TiO}_{2}$ Hollow Spheres Synthesized by One-pot Hydrothermal Method for Dye-sensitized Solar Cell Application, Energy Environ. Sci., 2011, 4, 3565-3572.

210 J.-F. Qian, P. Liu, Y. Xiao, Y. Jiang, Y.-L. Cao, X.-P. Ai and H.-X. Yang, $\mathrm{TiO}_{2}$-Coated Multilayered $\mathrm{SnO}_{2}$ Hollow Microspheres for Dye-sensitized Solar Cells, Adv. Mater., 2009, 21, 3663-3667.

211 E. J. Canto-Aguilar, M. Rodríguez-Perez, R. GarcíaRodríguez, F. I. Lizama-Tzec, A. T. D. Denko, F. E. Osterloh and G. Oskam, ZnO-based Dye-sensitized Solar Cells: Effects of Redox Couple and Dye Aggregation, Electrochim. Acta, 2017, 258, 396-404.

212 R. Ruess, S. Haas, A. Ringleb and D. Schlettwein, DyeSensitized Solar Cells with Electrodeposited $\mathrm{ZnO}$ and Co(bpy)3 Redox Electrolyte: Investigation of Mass Transport in the Electrolyte andInterfacial Charge Recombination, Electrochim. Acta, 2017, 258, 591-598.

213 S. V. Umale, S. N. Tambat and S. M. Sontakke, Combustion Synthesized $\mathrm{CeO}_{2}$ as an Anodic Material in Dye Sensitized Solar Cells, Mater. Res. Bull., 2017, 94, 483-488.

214 J.-Y. Bai, X.-L. Sun, G. Han and G.-W. Diao, Double-shell $\mathrm{CeO}_{2} @ \mathrm{TiO}_{2}$ Hollow Spheres Composites with Enhanced Light Harvesting and Electron Transfer in Dye-Sensitized Solar Cells, J. Alloys Compd., 2017, 722, 864-871.

215 R.-F. Zhao, Q.-H. Wu, D.-M. Tang, W.-L. Li, X. Zhang, M. Chen, R. Guo and G.-W. Diao, Double-Shell $\mathrm{CeO}_{2}: \mathrm{Yb}$, 
Er@SiO2@Ag Upconversion Composite Nanofibers as an Assistant Layer Enhanced near-infrared Harvesting for Dye-Sensitized Solar Cells, J. Alloys Compd., 2018, 769, 92-95.

216 G. Han, M. Wang, D.-Y. Li, J.-Y. Bai and G.-W. Diao, Novel Upconversion Er, $\mathrm{Yb}-\mathrm{CeO}_{2}$ Hollow Spheres as Scattering Layer Materials for Efficient Dye-sensitized Solar Cells, Sol. Energy Mater. Sol. Cells, 2017, 160, 54-59.

217 R.-F. Zhao, L. Huan, P. Gu, R. Guo, M. Chen and G.-W. Diao, Yb,Er-Doped $\mathrm{CeO}_{2}$ Nanotubes as an Assistant Layer for Photoconversion-enhanced Dye-sensitized Solar Cells, J. Power Sources, 2016, 331, 527-534.

218 M. Waqas, Y.-Z. Wei, D. Mao, J. Qi, Y. Yang, B. Wang and D. Wang, Multi-shelled $\mathrm{TiO}_{2} / \mathrm{Fe}_{2} \mathrm{TiO}_{5}$ Heterostructured Hollow Microspheres for Enhanced Solar Water Oxidation, Nano Res., 2017, 10, 3920-3928.

219 J. Qi, K. Zhao, G.-D. Li, Y. Gao, H.-J. Zhao, R.-B. Yu and Z.-Y. Tang, Multi-shelled $\mathrm{CeO}_{2}$ Hollow Microspheres as Superior Photocatalysts for Water Oxidation, Nanoscale, 2014, 6, 4072-4077.

220 Y.-Z. Wei, J.-Y. Wang, R.-B. Yu, J.-W. Wan and D. Wang, Constructing $\mathrm{SrTiO}_{3}-\mathrm{TiO}_{2}$ Heterogeneous Hollow Multishelled Structures for Enhanced Solar Water Splitting, Angew. Chem., 2019, 131, 1436-1440.

221 L.-L. Wang, Z. Lou, T. Fei and T. Zhang, Zinc Oxide CoreShell Hollow Microspheres with Multi-shelled Architecture for Gas Sensor Applications, J. Mater. Chem., 2011, 21, 19331-19336.

222 F.-D. Qu, W.-N. Shang, D.-T. Wang, S.-Y. Du, T. Thomas, S.-P. Ruan and M.-H. Yang, Coordination Polymer-Derived Multishelled Mixed Ni-Co Oxide Microspheres for Robust and Selective Detection of Xylene, ACS Appl. Mater. Interfaces, 2018, 10, 15314-15321.

223 J.-S. Kim, J.-W. Yoon, Y. J. Hong, Y. C. Kang, F. Abdel-Hady, A. Wazzan and J.-H. Lee, Highly Sensitive and Selective Detection of Ppb-Level $\mathrm{NO}_{2}$ using Multi-shelled $\mathrm{WO}_{3}$ Yolk-shell Spheres, Sens. Actuators, B, 2016, 229, 561-569.

224 Y.-J. Li, S.-M. Wang, P. Hao, J. Tian, H.-Z. Cui and X.-Z. Wang, Soft-Templated Formation of Double-shelled ZnO Hollow Microspheres for Acetone Gas Sensing at Low Concentration/near Room Temperature, Sens. Actuators, B, 2018, 273, 751-759.

225 J.-Z. Zheng, T.-M. Zhang, H.-J. Zeng, W. Guo, B. Zhao, Y.-H. Sun, Y.-Y. Li and L. Jiang, Multishelled Hollow Structures of Yttrium Oxide for the Highly Selective and Ultrasensitive Detection of Methanol, Small, 2019, 15, 1804688.

226 Y. J. Hong, J.-W. Yoon, J.-H. Lee and Y. C. Kang, One-Pot Synthesis of Pd-Loaded $\mathrm{SnO}_{2}$ Yolk-shell Nanostructures for Ultraselective Methyl Benzene Sensors, Chem. - Eur. J., 2014, 20, 2737-2741.

227 S.-J. Kim, I.-S. Hwang, C. W. Na, I.-D. Kim, Y. C. Kang and J.-H. Lee, Ultrasensitive and Selective $\mathrm{C}_{2} \mathrm{H}_{5} \mathrm{OH}$ Sensors using Rh-loaded $\mathrm{In}_{2} \mathrm{O}_{3}$ Hollow Spheres, J. Mater. Chem., 2011, 21, 18560-18567.

228 N. G. Cho, H.-S. Woo, J.-H. Lee and I.-D. Kim, Thin-Walled NiO Tubes Functionalized with Catalytic Pt for Highly
Selective $\mathrm{C}_{2} \mathrm{H}_{5} \mathrm{OH}$ Sensors using Electrospun Fibers as a Sacrificial Template, Chem. Commun., 2011, 47, 11300-11302.

229 J.-W. Yoon, Y. J. Hong, G. D. Park, S.-J. Hwang, F. AbdelHady, A. A. Wazzan, Y. C. Kang and J.-H. Lee, Kilogramscale Synthesis of Pd-Loaded Quintuple-Shelled $\mathrm{Co}_{3} \mathrm{O}_{4}$ Microreactors and Their Application to Ultrasensitive and Ultraselective Detection of Methylbenzenes, ACS Appl. Mater. Interfaces, 2015, 7, 7717-7723.

230 P. Sun, B.-Q. Wang, L.-P. Zhao, H.-Y. Gao, T.-S. Wang, X.-L. Yang, C. Liu and G.-Y. Lu, Enhanced Gas Sensing by Amorphous Double-shell $\mathrm{Fe}_{2} \mathrm{O}_{3}$ Hollow Nanospheres Functionalized with PdO Nanoparticles, Sens. Actuators, B, 2017, 252, 322-329.

231 T.-T. Ma, L.-L. Zheng, Y.-Q. Zhao, Y.-S. Xu, J. Zhang and X.-H. Liu, Highly Porous Double-shelled Hollow Hematite Nanoparticles for Gas Sensing, ACS Appl. Nano Mater., 2019, 2, 2347-2357.

232 X.-M. Ma, X.-T. Zhang, L. Yang, K. Wang, K. Jiang, Z.-P. Wei and Y.-M. Guo, An Unusual Temperature Gradient Crystallization Process: Facile Synthesis of Hierarchical ZnO Porous Hollow Spheres with Controllable Shell Numbers, CrystEngComm, 2014, 16, 7933-7941.

233 D.-F. Zhang, G.-Z. Zhang and L. Zhang, Multi-Shelled $\mathrm{FeCo}_{2} \mathrm{O}_{4}$ Hollow Porous Microspheres/CCFs Magnetic Hybrid and its Dual-Functional Catalytic Performance, Chem. Eng. J., 2017, 330, 792-803.

234 L.-B. Zong, P.-Z. Cui, F.-Y. Qin, K. Zhao, Z.-M. Wang and R.-B. Yu, Heterostructured Bismuth Vanadate Multi-shell Hollow Spheres with High Visible-Light-Driven Photocatalytic Activity, Mater. Res. Bull., 2017, 86, 44-50.

235 F.-Y. Qin, P.-Z. Cui, L. Hu, Z.-M. Wang, J. Chen, X.-R. Xing, H. Wang and R.-B. Yu, Construction of Multi-shelled $\mathrm{Bi}_{2} \mathrm{WO}_{6}$ Hollow Microspheres with Enhanced Visible Light Photo-Catalytic Performance, Mater. Res. Bull., 2018, 99, 331-335.

236 B.-J. Liu, X.-Y. Li, Q.-D. Zhao, Y. Hou and G.-H. Chen, Selftemplated Formation of $\mathrm{ZnFe}_{2} \mathrm{O}_{4}$ Double-shelled Hollow Microspheres for Photocatalytic Degradation of Gaseous O-Dichlorobenzene, J. Mater. Chem. A, 2017, 5, 8909-8915.

237 K.-L. Ma, W.-X. Zou, L. Zhang, L.-L. Li, S.-H. Yu, C.-J. Tang, F. Gao and L. Dong, Construction of Hybrid Multi-shell Hollow Structured $\mathrm{CeO}_{2}-\mathrm{MnO}_{x}$ Materials for Selective Catalytic Reduction of NO with $\mathrm{NH}_{3}, R S C A d v$., 2017, 7, 5989-5999.

238 H. Wang, D. Mao, J. Qi, Q.-H. Zhang, X.-H. Ma, S.-Y. Song, L. Gu, R.-B. Yu and D. Wang, Hollow Multishelled Structure of Heterogeneous $\mathrm{Co}_{3} \mathrm{O}_{4}-\mathrm{CeO}_{2-x}$ Nanocomposite for $\mathrm{CO}$ Catalytic Oxidation, Adv. Funct. Mater., 2019, 29, 1806588.

239 L. Wang, J.-W. Wan, Y.-S. Zhao, N.-L. Yang and D. Wang, Hollow Multi-shelled Structures of $\mathrm{Co}_{3} \mathrm{O}_{4}$ Dodecahedron with Unique Crystal Orientation for Enhanced Photocatalytic $\mathrm{CO}_{2}$ Reduction, J. Am. Chem. Soc., 2019, 141, 2238-2241.

240 X. Wang, Y.-T. Zhong, T.-Y. Zhai, Y.-F. Guo, S.-M. Chen, Y. Ma, J.-N. Yao, Y. Bando and D. Golberg, Multishelled $\mathrm{Co}_{3} \mathrm{O}_{4}-\mathrm{Fe}_{3} \mathrm{O}_{4}$ Hollow Spheres with Even Magnetic Phase 
Distribution: Synthesis, Magnetic Properties and their Application in Water Treatment, J. Mater. Chem., 2011, 21, 17680-17687.

241 Y. Suzuki, S. D. Kelly, K. M. Kemner and J. F. Banfield, Nanometre-size Products of Uranium Bioreduction, Nature, 2002, 419, 134.

242 J. Li, X.-X. Wang, G.-X. Zhao, C.-L. Chen, Z.-F. Chai, A. Alsaedi, T. Hayat and X.-K. Wang, Metal-organic Framework-based Materials: Superior Adsorbents for the Capture of Toxic and Radioactive Metal Ions, Chem. Soc. Rev., 2018, 47, 2322-2356.

243 Y.-J. Ai, Y. Liu, W.-Y. Lan, J.-R. Jin, J.-L. Xing, Y.-D. Zou, C.-F. Zhao and X.-K. Wang, The Effect of $\mathrm{pH}$ on the U(vI) Sorption on Graphene Oxide (GO): A Theoretical Study, Chem. Eng. J., 2018, 343, 460-466.

244 X.-X. Wang, S.-Q. Yu, Y.-H. Wu, H.-W. Pang, S.-J. Yu, Z.-S. Chen, J. Hou, A. Alsaedi, T. Hayat and S.-H. Wang, The Synergistic Elimination of Uranium(vI) Species from Aqueous Solution using Bi-Functional Nanocomposite of Carbon Sphere and Layered Double Hydroxide, Chem. Eng. J., 2018, 342, 321-330.

245 S. Song, S. Zhang, S.-Y. Huang, R. Zhang, L. Yin, Y.-Z. Hu, T. Wen, L. Zhuang, B.-W. Hu and X.-K. Wang, A Novel Multi-Shelled $\mathrm{Fe}_{3} \mathrm{O}_{4} @ \mathrm{MnO}_{x}$ Hollow Microspheres for Immobilizing U(VI) and Eu(III), Chem. Eng. J., 2019, 355, 697-709.

246 J.-Q. Feng, H.-X. Guo, S.-P. Wang, Y.-J. Zhao and X.-B. Ma, Fabrication of Multi-shelled Hollow Mg-Modified $\mathrm{CaCO}_{3}$ Microspheres and their Improved $\mathrm{CO}_{2}$ Adsorption Performance, Chem. Eng. J., 2017, 321, 401-411.
247 B.-C. Liu, Q. Wang, S.-L. Yu, T. Zhao, J.-X. Han, P. Jing, W.-T. Hu, L.-X. Liu, J. Zhang, L.-D. Sun and C.-H. Yan, Double Shelled Hollow Nanospheres with Dual Noble Metal Nanoparticle Encapsulation for Enhanced Catalytic Application, Nanoscale, 2013, 5, 9747-9757.

248 P.-F. Xu, R.-B. Yu, H. Ren, L.-B. Zong, J. Chen and X.-R. Xing, Hierarchical Nanoscale Multi-shell $\mathrm{Au} / \mathrm{CeO}_{2}$ Hollow Spheres, Chem. Sci., 2014, 5, 4221-4226.

249 Y.-Y. Liao, Y. Li, L. Wang, Y.-X. Zhao, D.-Y. Ma, B.-Q. Wang, Y.-X. Wan and S.-L. Zhong, Multi-Shelled Ceria Hollow Spheres with a Tunable Shell Number and Thickness and their Superior Catalytic Activity, Dalton Trans., 2017, 46, 1634-1644.

250 C. Zhang, Y.-M. Zhou, Y.-W. Zhang, S. Zhao, J.-S. Fang and X.-L. Sheng, In Situ Doping of Pt Active Sites via Sn in Double-Shelled $\mathrm{TiO}_{2}$ Hollow Nanospheres with Enhanced Photocatalytic $\mathrm{H}_{2}$ Production Efficiency, Chem. - Eur. J., 2017, 41, 11089-11096.

251 P. K. Bollaa, V. A. Rodrigueza, R. S. Kalhapurea, C. S. Kollib, S. Andrewsa and J. Renukuntla, A Review on $\mathrm{pH}$ and Temperature Responsive Gels and Other Less Explored Drug Delivery Systems, J. Drug Delivery Sci. Technol., 2018, 46, 416-435.

252 M. Shahriari, M. Zahiric, K. Abnousa, S. M. Taghdisid, M. Ramezani and M. Alibolandi, Enzyme Responsive Drug Delivery Systems in Cancer Treatment, J. Controlled Release, 2019, 308, 172-189.

253 C.-C. Huang, W. Huang and C.-S. Yeh, Shell-By-Shell Synthesis of Multi-shelled Mesoporous Silica Nanospheres for Optical Imaging and Drug Delivery, Biomaterials, 2011, 32, 556-564. 